DOE/ET/20175-1

\title{
Solar Radiation Observation Stations Updated to 1979
}

April 1979

Prepared for:

U.S. Department of Energy

Assistant Secretary for Energy Technology

Division of Distributed Solar Technology

Contract No. DE-AS05-78ET-20175 


\section{DISCLAIMER}

This report was prepared as an account of work sponsored by an agency of the United States Government. Neither the United States Government nor any agency Thereof, nor any of their employees, makes any warranty, express or implied, or assumes any legal liability or responsibility for the accuracy, completeness, or usefulness of any information, apparatus, product, or process disclosed, or represents that its use would not infringe privately owned rights. Reference herein to any specific commercial product, process, or service by trade name, trademark, manufacturer, or otherwise does not necessarily constitute or imply its endorsement, recommendation, or favoring by the United States Government or any agency thereof. The views and opinions of authors expressed herein do not necessarily state or reflect those of the United States Government or any agency thereof. 


\section{DISCLAIMER}

Portions of this document may be illegible in electronic image products. Images are produced from the best available original document. 
Available from:

National Technical Information Service (NTIS)

U.S. Department of Commerce

5285 Port Royal Road

Springfield, Virginia 22161

Price: Printed copy: $\$ 6.50$

Microfiche: $\quad \$ 3.00$ 


\section{Solar Radiation Observation Stations Updated to 1979}

Prepared by: The Kenneth E. Johnson Environmental and Energy Center

Huntsville, Alabama

Contract No. DE-AS05-78ET-20175 .

Prepared for:

U.S. Department of Energy.

Assistant Secretary for Energy Technology

Division of Distributed Solar Technology

Washington, D. C. 20585

United States nor the United States Depany of their

Energy. nor any of their employees, her any or their

contractors, subcontractor, or their er assumes any lega

any warranty, express or implied, or assumes completentss

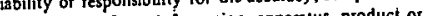

of usefulness of any information, apparatus, produch of
process disclosed, or represents that its use would not

infringe privately owned rights. 


\section{NOTICE}

This report wes prepered as an account of work sponsored by the United States Government. Neither the United States nor the United States Department of Energy, nor any of their employees, makes any warranty, express or implied, or asumes any logal liability or resoonsibility for the accuracy, completeness. Or usefulness of any information, apparatus, product, or process disclosed, or represents that its use would not infringe privately owned rights. Reference herein to any specific commercial product, process, or service by trede name, mark, manufacturer, or otherwise, does not necessarily constitute or imply its endorsement, reoommendetion, or fevoring by the United States Government or any soency thereof. The views and opinions of w thors expressed herein do not necesisarily state or reflect those of the United Stetes Government or any epency thereot. 
This report presents the results of research performed by the Kenneth E. Johnson Environmental and Energy Center (JEEC) at the University of Alabama in Huntsville (UAH) for the Department of Energy, Division of Solar Technology, Environmental Planning and Analysis Branch and is a follow-up to an earlier report entitled "Solar Radiation Observation Stations with Complete Listing of Data Archived by the National Climatic Center, Asheville, North Carolina, and Initial Listing of Data not Currently Archived." ERDA (1976). .

This document is a catalog of the solar radiation observation stations in the U.S. and includes the type of equipment used in measuring and recording the observations. The main emphases are on the locations and types of solar radiation observations, the need to examine data carefully before application to a project and the need to increase the nation's solar radiation data base.

With the present interest in solar energy, the location and status of solar radiation measuring stations are changing, and comments and suggestions concerning this report are requested from the reader.

Acknowledgement

The authors acknowledge the help and cooperation from many organizations listed herein and appreciate the willing support of individuals who provided information for this report. Particular thanks are expressed to Mr. Michael Riches of the Department of Energy, Division of Solar Technology, Environmental Planning and Analysis Branch and Mr. Frank Quinlan of the National Oceanic and Atmospheric Administration, Environmental Data and Information Service, National Climatic Center, for their contributions to this report and to secretaries Sherri Higgins and Valerie Seaquist for their assistance.

Eugene A. Carter

Jacqueline R. Cristina

Baker B. Williams 


\section{TABLE OF CONTENTS}

1. Introduction ................... I

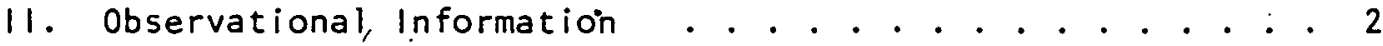

National Weather Service .......... 2

Department of Energy ............ 2

State Organizations ............ 3

Power Companies ............. . . 3

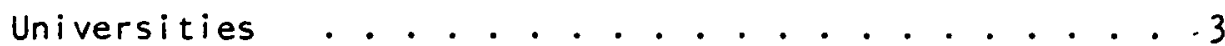

National Solar Heating and Cooling

Demonstration Program ............ 3

other Organizations . . . . . . . . . . . 4

Locations of Solar Radiation Stations ........ 5

111. Publications . . . . . . . . . . 5

IV. Summary and Conclusions ........... 5 
FIGURES

Figure 1 Solar Radiation Stations With Data Archived at the National Climatic Center, Asheville, North Carolina. Numbers Indicate More Than One Station in Area.

Figure 2 Stations With Rehabilitated and Derived Global Solar Radiation. Asterisks Indicate Stations With Data Rehabilitated.

Figure 3 Up-dated National Weather Service Solar Radiation Station Network.

Fiqure 4 Solar Kadiation Stations Nor in The Baslc Natiund Weather Service, NOAA, Network. Asterisks Denote DOE Research and Training Sites. Numbers Indicate More Than One Station in The Area.

Figure 5 Non-NOAA Stations Which Recorded Solar Radiation in the Past But Were Not Recording Solar Radiation in December 1978.

Figure 6 Location of National Solar Heating and Cooling Demonstration Sites. 


\section{APPENDI.CES}

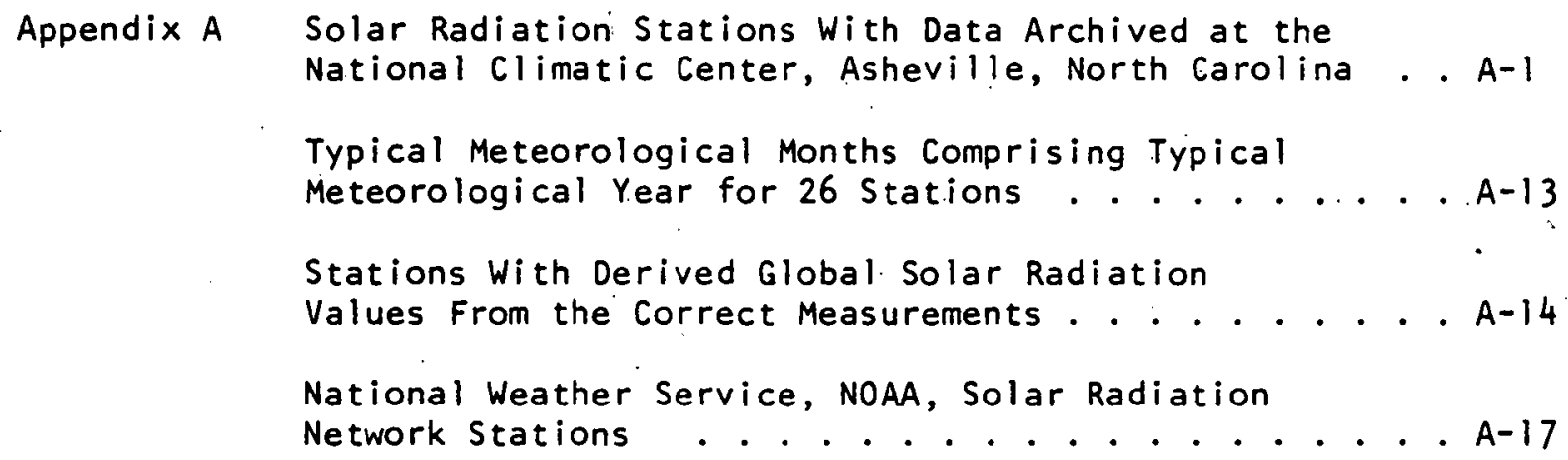

Appendix B Solar Radiation Stations Not in the Basic
National Weather Service, NOAA, Network ....... B-1

Appendix C. Publications With Solar Radiation Data.......... C-l

Appendix $D$ Department of Energy National Solar Heating and Cooling Demonstration Sites ........... D-1

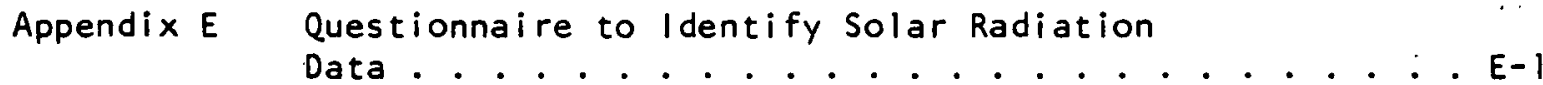




\section{INTRODUCTION}

The main emphasis of an earlier report, ERDA (1976), was to inform those interested in solar energy projects of the locations where solar radiation data were available. This document is directed toward identifying the locations and types of solar radiation observations presently being taken, the data archived at the National Climatic Center (NCC) which have been rehabilitated, and the radiation data continually being processed by the NCC. The earlier publication listed 316 stations where solar radiation measurements were made, and this document includes 420 stations in the United States where solar radiation measurements were being taken as of January 1, 1979.

This report does not attempt to evaluate solar radiation data nor confirm the validity of any information provided by the various sources. It lists the type of sensing and recording equipment at each station, and the quality of the equipment may be an indication of the quality of the measurements. For information on solar radiation measuring equipment see ERDA (1977) and for information on the taking of solar radiation observations see Carter, et al, (1978) and International Energy Agency (I.E.A.) Handbook (1979). From January 1, 1957 to 1977 the International Pyrheliometric Scale, IPS 1956, was used in solar radiation measurements. In 1977 and 1978 many stations changed to the Absolute Scale which gives measurements near two:percent higher than the IPS 1956.

The solar radiation stations are listed alphabetically by states. Reports of summarized solar radiation data encountered during the preparation of this document are listed in Appendix $C$. When a publication contains radiation data or related computations of radiation for a location, the location has a number in parenthesis, under the record form column, corresponding to the number of the publication in Appendix $C$.

With the increasing interest in the use of solar energy systems, many changes are occurring in station locations, observational equipment and recording techniques for solar radiation measurements. It is hoped that those interested will provide any needed changes, comments and additional data to keep this report up-to-date. Thereby, the technical community may learn of the existence of solar radiation observations and possibly avoid expensive and time consuming duplications of such research activities. 


\section{OBSERVATIONAL INFORMATION}

\section{National Weather Service -}

The information on the solar radiation observations made by the National Weather Service (NWS) of the National Oceanic and Atmospheric Administration (NOAA) was obtained from the National Climatic Center (NCC), Environmental Data and Information Service, (EDIS), NOAA. The NWS data provide the most complete observations and the greatest geographical distribution of solar radiation measurements available in the country (Figures 1,2 and 3 and Appendix A). The data are stored on magnetic tape and are available for purchase from the NCC, see SOLMET 1978. The data from the stations 1 isted in $A-17$ are available in hourly values in the publication Monthly Summary - Solar Radiatinn nata from NCC, Ashevilie, North Carolina.

The updated NOAA solar radiation network (Figure 3) began operating in 1977 with the support of the U.S. Energy Research and Development Administration (ERDA), Division of Solar Technology. The ERDA became part of the U.S. Department of Energy (DOE) in October 1977.

The ERDA, Division of Solar Technology, assisted the NCC in rehabilitating archived NWS solar radiation data. The rehabilitated stations are listed in Appendix $A-13$ and the station locations are indicated in Figure 2. Standard solar radiation years have been established for the locations of the rehabilitated stations, Appendix $A-13$. Stations with derived solar radiation values are indicated on Figure 2 and listed in Appendix A-14.

\section{Department of Energy -}

The DOE through its. Enviranmental and Resource Assessment Branch, Division of Solar Technology, established eight university meteorological. research and training sites. The sites are located to serve regions of the U.S., see Figure 4 and Appendix 8 . The multiple types of observations and the training at these sites will vary, but will be directed toward developing, the use of solar energy in the region.

The DOE is assisting in the collection of solar radiation data from universities, power companies and other cooperators with data which meet or exceed the quality standard set for NWS, NOAA. Other laboratories of the DOE are taking a variety of solar radiation measurements and are indicated in the listings in Appendix B. 
State Organizations -

Some. states have organized a solar radiation observation network to obtain radiation data for energy planning and other research projects. A few of the states and the departments involved in solar energy and related research projects follow:

California - Department of Energy and Department of Water Resources Hawai - Department of Planning and Economic Development

Montana - Department of Natural Resources and Conservation

Some State Climatologists and universities are collecting solar radiation measurements and publishing the data, see Appendix $C$.

Power Companies -

Many power companies have developed a solär radiation measuring network, and a few have formed a consortium to collect radiation data from states in a region. The Northeast Utilities collect data from the southern New England states. San Diego Gas and Electric Company obtains data from San Diego and surrounding California counties. The Tennessee Valley Authority has one of the oldest solar radiation networks in the U.S. with solar radiation and meteorological data from Tennessee, Alabama and Kentucky, and two stations have been in operation for twelve years. The Western Supply and Transmission (WEST) Associates is an organization of about twenty power and service companies and collect solar radiation data from five southwestern states.

\section{Universities -}

Solar radiation measurements by various university departments have been made for a variety of uses, from instruction to research. The records, therefore, vary widely in quality. Those supporting agriculture experiment stations were the most consistent, although their equipment was not intended for high resolution.

National Solar Heating and Cooling Demonstration Program -

To collect and analyze data which is necessary for the DOE to determine the thermal performance of selected solar energy systems, the Federal Systems Division of the International Business Machines Corporation (IBM), under a DOE contract, has established a national solar data network (Figure 6 ). The data include direct, plus diffuse radiation on a tilted surface and ambient air temperature for about 81 sites in the U.S. Some sites also report diffuse solar radiation on a tilted surface, wind speed, wind direction and relative humidity. 
Appendix $D$ lists the National Solar. Heating and Cooling Bemonstration Program. (N.S:H.C.D.P.) stations.

\section{Other Organizations -}

Other government and private organizations recorded solar radiation measurements, but generally, these observations were made to serve a specific program and may not be usable for other purposes.

The National Environmental Protection Agency and a few state and local environmental protection agencies have measured solar radiation because of its role in the formation of photochemical smog. Some of these measurements recorded only ultraviolet radiation, wl th wavelengths of approximately $0.295-0.385 \mathrm{micro-}$ meters $(\mu \mathrm{m})$.

Health organizations and private companies measured the ultraviolet radiation to determine its effect on humans and materials.

Since the energy from the sun is the producer of weather and climates, some organizations compiled electronic tapes with weather and solar radiation data for special uses such as input for computer simulations.

Other organizations identified as having recorded solar radiation data for specific test purposes are:

1) Bigelow Laboratory for Ocean Sciences

2). Desert Sunshine Exposure Tests, Inc.

3) Eppley Laboratories

4) National Aeronautics and Space Administration

5) Scripps Institute of Oceanography

6) Smithsonian Institute

7) U.S. Dureou of Reclallid LIun

8) U.S. Department of Defense

9) U.S. Forest Service

10) U.S. Geological Survey

Many of the stations. Iisted in Appendix $B$ recorded solar radiation data for special use categories and care should be exercised in selecting data which may be applicable to a project. 


\section{Locations of Solar Radiation Stations -}

Solar radiation stations which are not systematically forwarding their data to the NCC are indicated in Figure 4. Information on these stations is given in Appendix $B$. The non-NWS stations which have taken solar radiation observations in the past but were not operating as of January 1, 1979, are indicated in Figure 5. The information on these stations is given in Appendix $B$. The NWS updated solar radiation stations are given in Figure 3 and Appendix A-17. These stations are equipped with pyranometers, Eppley PSP and/or. Spectrolab SR75, and a pyrheliometer, Eppley NIP.

\section{PUBLICATIONS}

Many state departments, power companies, universities, solar energy centers and others have published reports containing solar radiation data. The publications encountered in the preparation of this report are listed in Appendix $C$. If a station, which is listed in Appendix $B$, has its data in a publication, a number is in the record form column and the publication is identified by. that number. The publication listing is not comprehensive but is included for the user's convenience.

No criteria have been established for the publications listed, and the equipment and observational standards vary greatly for data in a publication. This report does not include evaluations of the published data. Nevertheless, some of the data in the listed publications would be adaptable to general use or preliminary planning for certain projects.

\section{SUMMARY AND CONCLUSIONS}

Appendix A includes information on 38 NOAA, NWS stations which are presently measuring global; direct and diffuse solar radiation, a listing of data which have been rehabilitated from 26 stations with a standard year established for hourly solar radiation and a listing of 222 stations which have derived global solar radiation values. Appendix A includes, also, a listing of NWS stations which recorded solar radiation measurements in the past.

Appendix $B$ includes a listing of 391 stations in the United States which have recorded solar radiation measurements and 30 of of these stations were in operation on January 1, 1979, for closed stations see Appendix $B$ and Figure 5.

To expand our knowledge of this basic energy resource and to establish the nations's radiation climatology, solar radiation measurements which meet the established standards need to be archived at the NCC. Considering the climatic zones of the nation, data from 
additional solar radiation stations to supplement the NWS data, should be added to the basic national data bank at the NCC.

Special requirements and some areas near large inland bodies of water, industrial sections, mountains or sea coasts will demand additional measurements, but once a standard radiation year is established for a location the measurements at that location could be deleted unless there were special demands such as testing the efficiency of a solar energy system, forecasting the available solar radiation or bench marks.

This report attempts to inform those in the research and engineering fields, who are interested in solar energy, of the locations of present solar radiation stations, the past historical records which are available, and the necessity to evaluate carefully the observational and data processing procedures used in the solar radiation measurements before applying the data to a project.

Due to the increasing interest in solar energy, changes are occurring in the locations of solar radiation stations, the types of observational equipment and recording techniques of radiation measurements. The assistance of the reader is respectfully requested in reporting these changes and in identifying any sources of solar radiation data not included in this report. A copy of a questionnaire form to report this information is included as Appendix E. A similar questionnaire was used to obtain the information on most of the stations in Appendix B.

By making the past records and present activity in solar radiation measurements available to the community active in solar energy projects, the duplication of efforts should be prevented, thereby saving money, manpower and materials. 


\section{REFERENCES}

Carter, E. A. and D. L. Christensen, B. B. Williams..(1978), "Solar Radiation Data Sources, Applications and Network Design," U.S. DOE, HCP/T5362-01.

ERDA (1976), "Solar Radiation Observation Stations With Complete Listing of Data Archived by the National Climatic Center, Asheville, North Carolina, and Initial Listing of Data Not Currently Archived," DSE/1024-2, NTIC, DOC.

ERDA (1977), "Catalog of Solar Radiation Measuring Equipment", DSE ORO/5362-1, NTIS, DOC.

International Energy Agency Handbook (1979) in preparation.

SOLMET (1978), "Hourly Solar Radiation - Surface Meteorological

' Observations, Volume 1, User's Manual, TD-9724, National Climatic Center, Asheville, North Carolina 28801. 


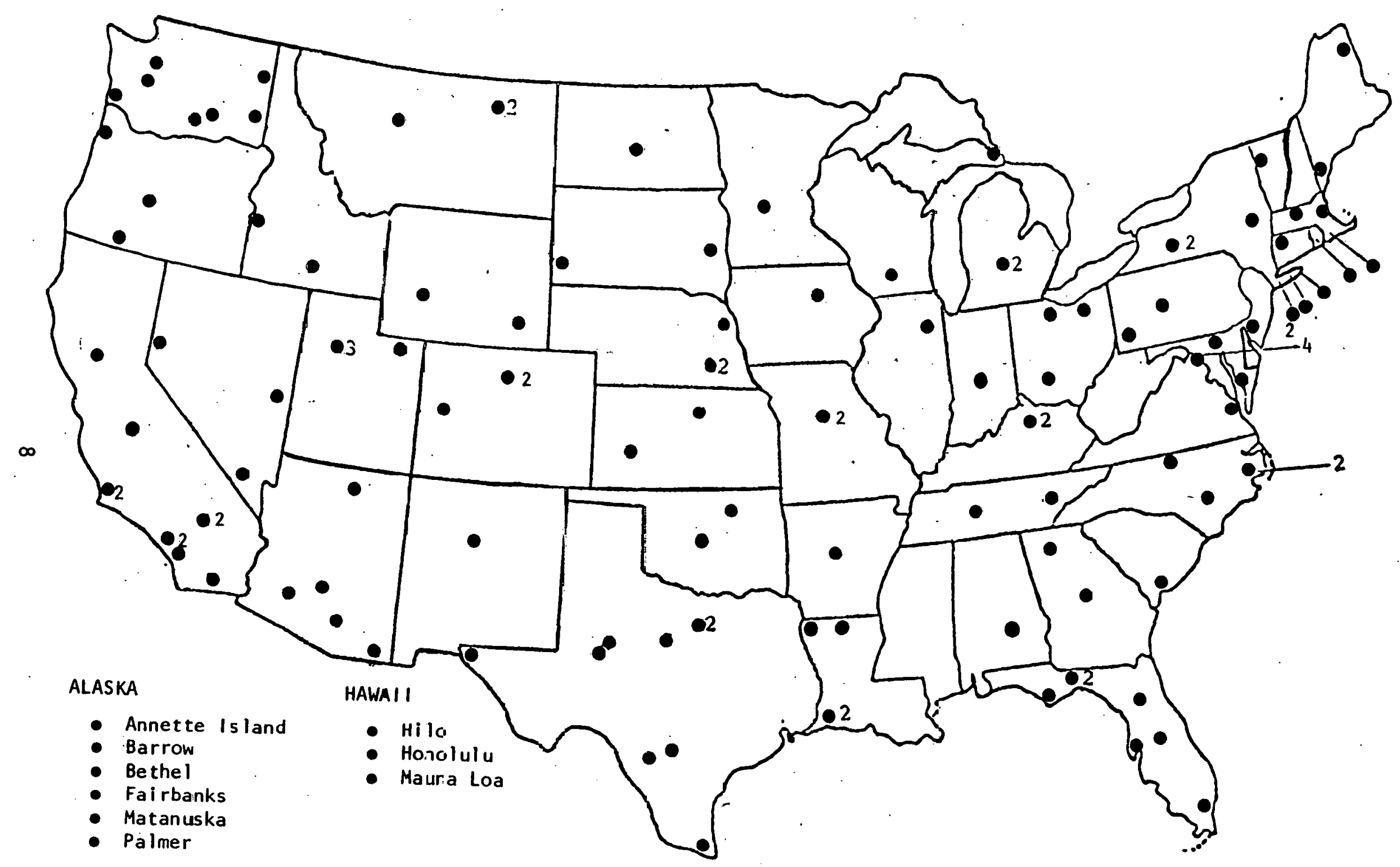

FIGURE I - SOLAR RADIATIDN STATIOHS WITH DATA ARCHIVED AT THE NATIONAL CLIMATIC CENTER, ASHEVILLE, NORTH CAROLINA. NUMBERS INDICATE MORE THAN ONE STATION IN AREA. 


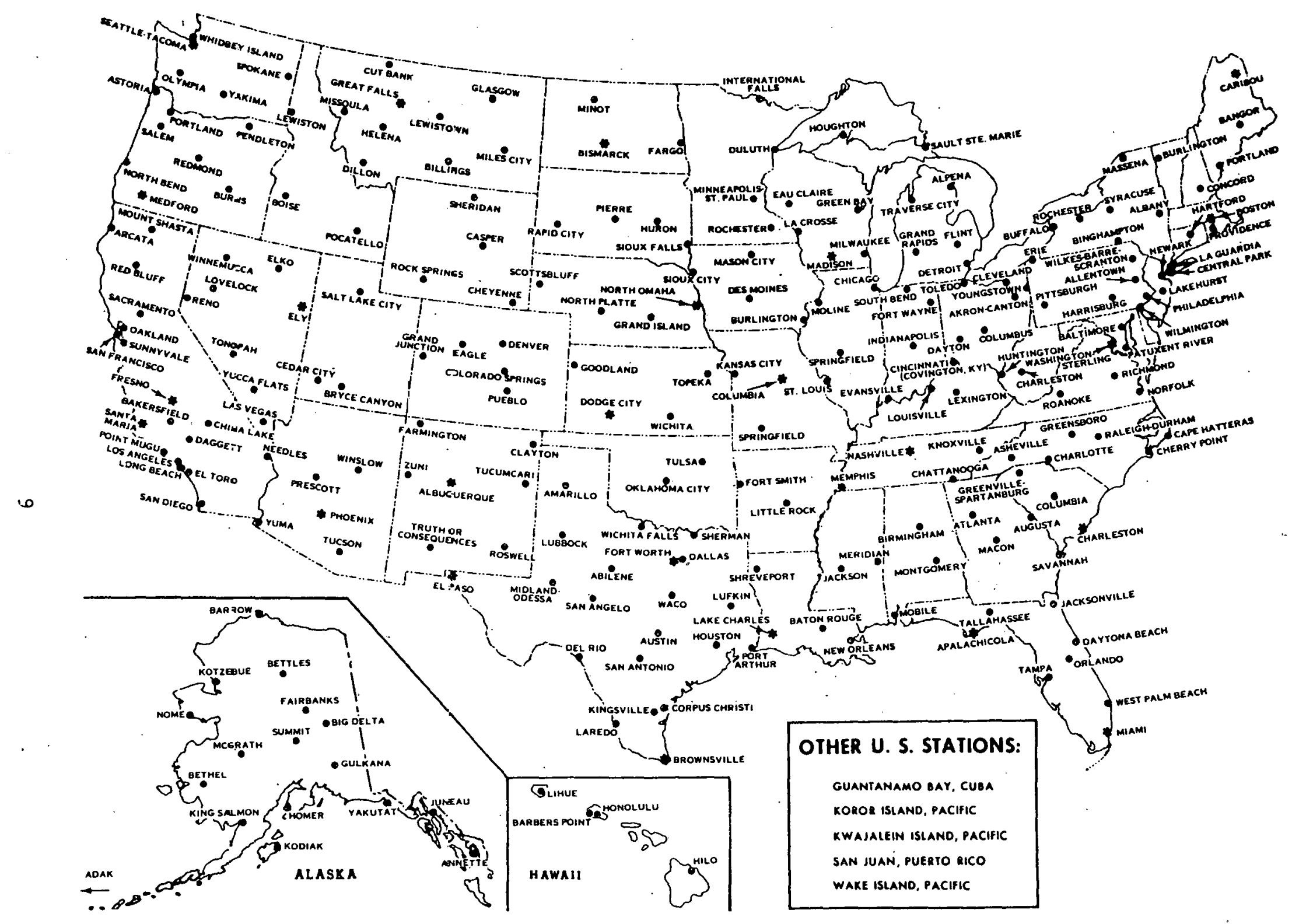

FIGURE 2 - STATIONS WITH REHABILITATED AND DERIVED GLOBAL SOLAR RADIATION. ASTERISKS INDICATE STATIONS WITH DATA REHABILITATED. 


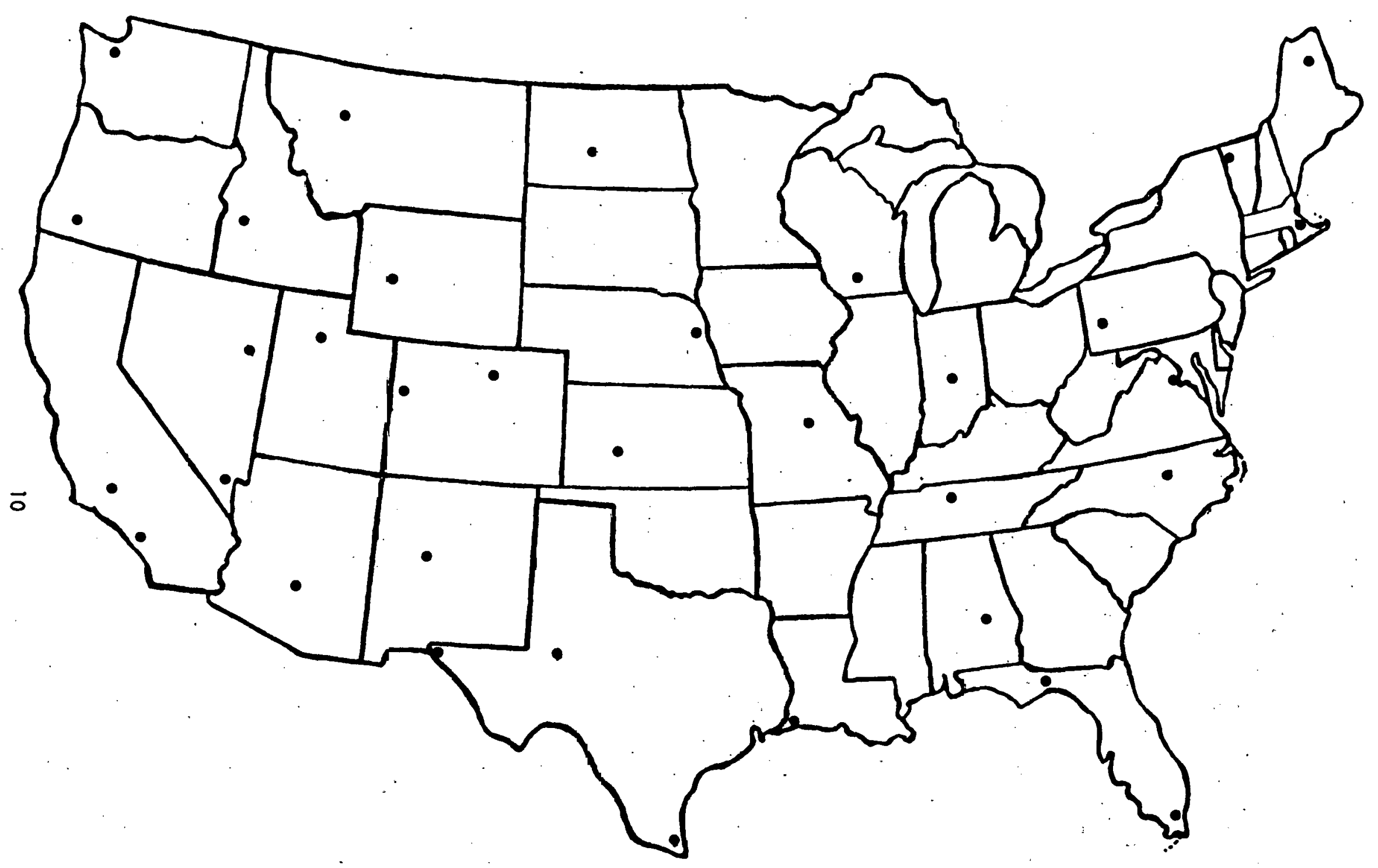

- Fairbanks, AK

- San Juan, F.R.

- Honolulu, HI

- Guam, M.I.

FIGURE 3 - UPDATED NATIONAL WEATHER SERVICE SOLAR RADIATION STATION NETWORK 


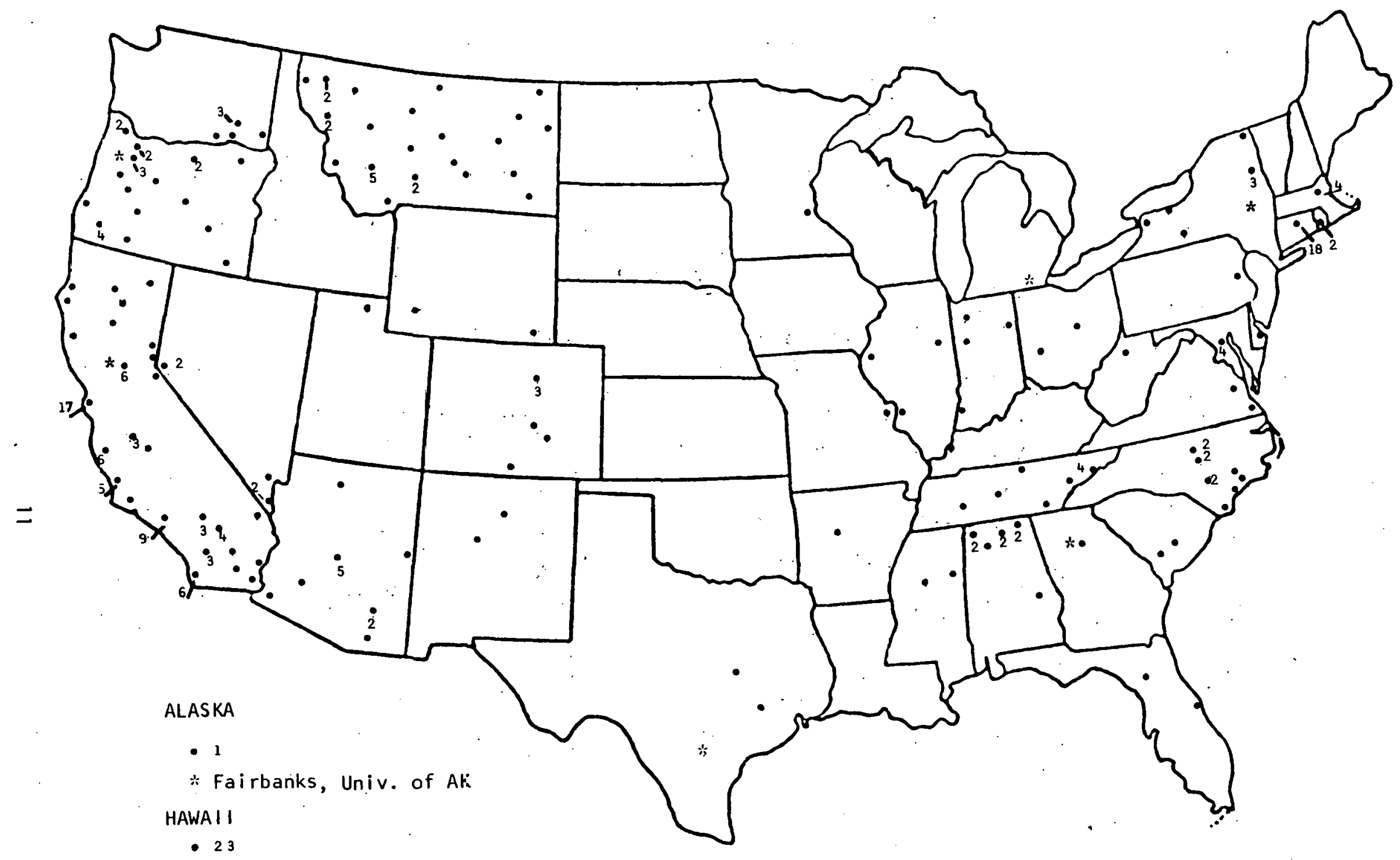

$*$ Univ. of $\mathrm{HI}$

FIGURE 4 - SOLAR RADIATION STATIONS NOT IN THE BASIC NATIONAL WEATHER SERVICE, NOAA, NETWORK. ASTERISKS DENOTE DOE RESEARCH AND TRAINING SITES. NUMBERS INDICATE MORE THAN ONE STATION IN THE AREA. 


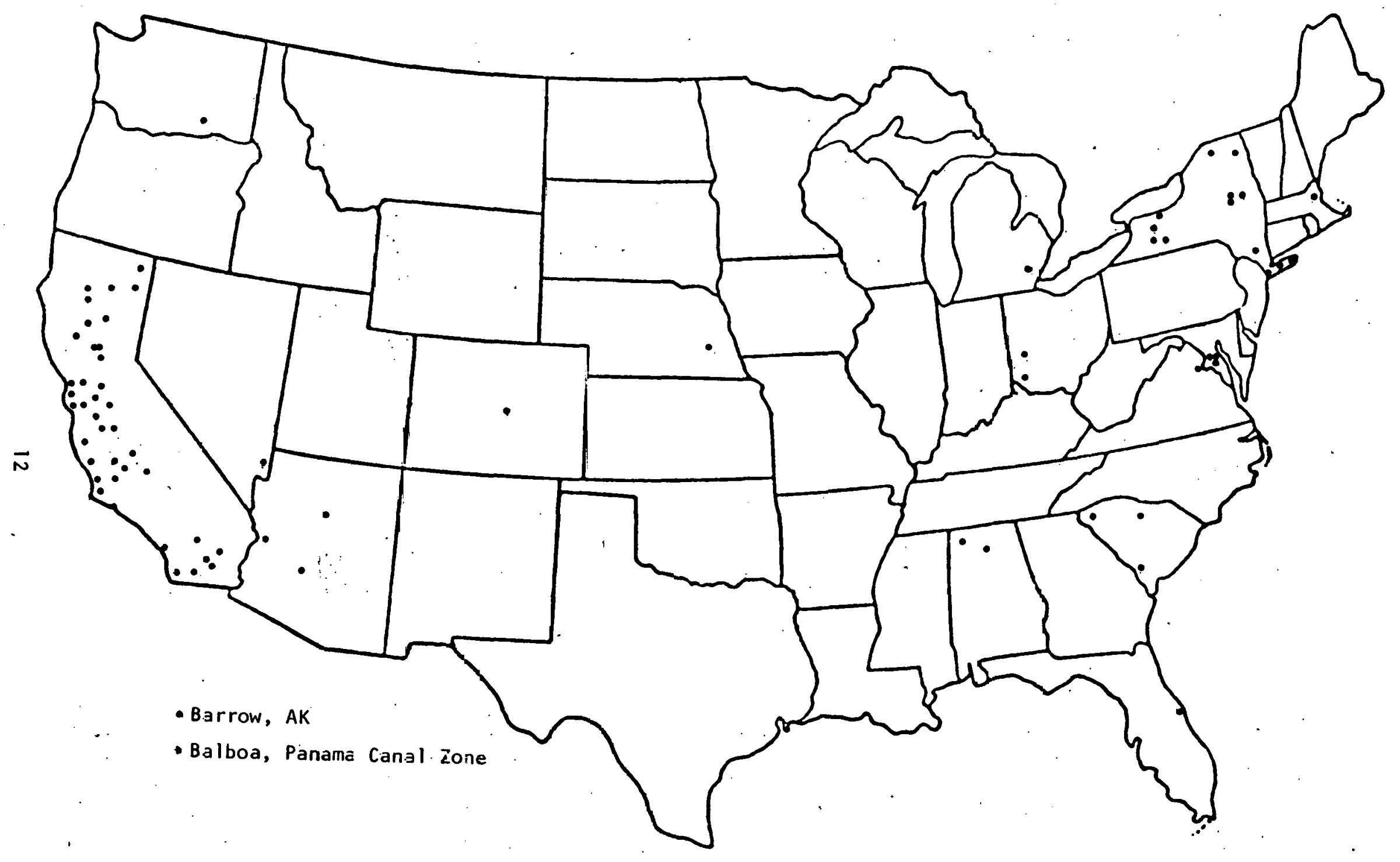

FIGURE 5 - NON-NOAA STATIONS WHICH RECORDED SOLAR RADIATION IN THE PAST BUT WERE NOT RECIJRDING SOLAR RADIATION IN DECEMBER 1978. 


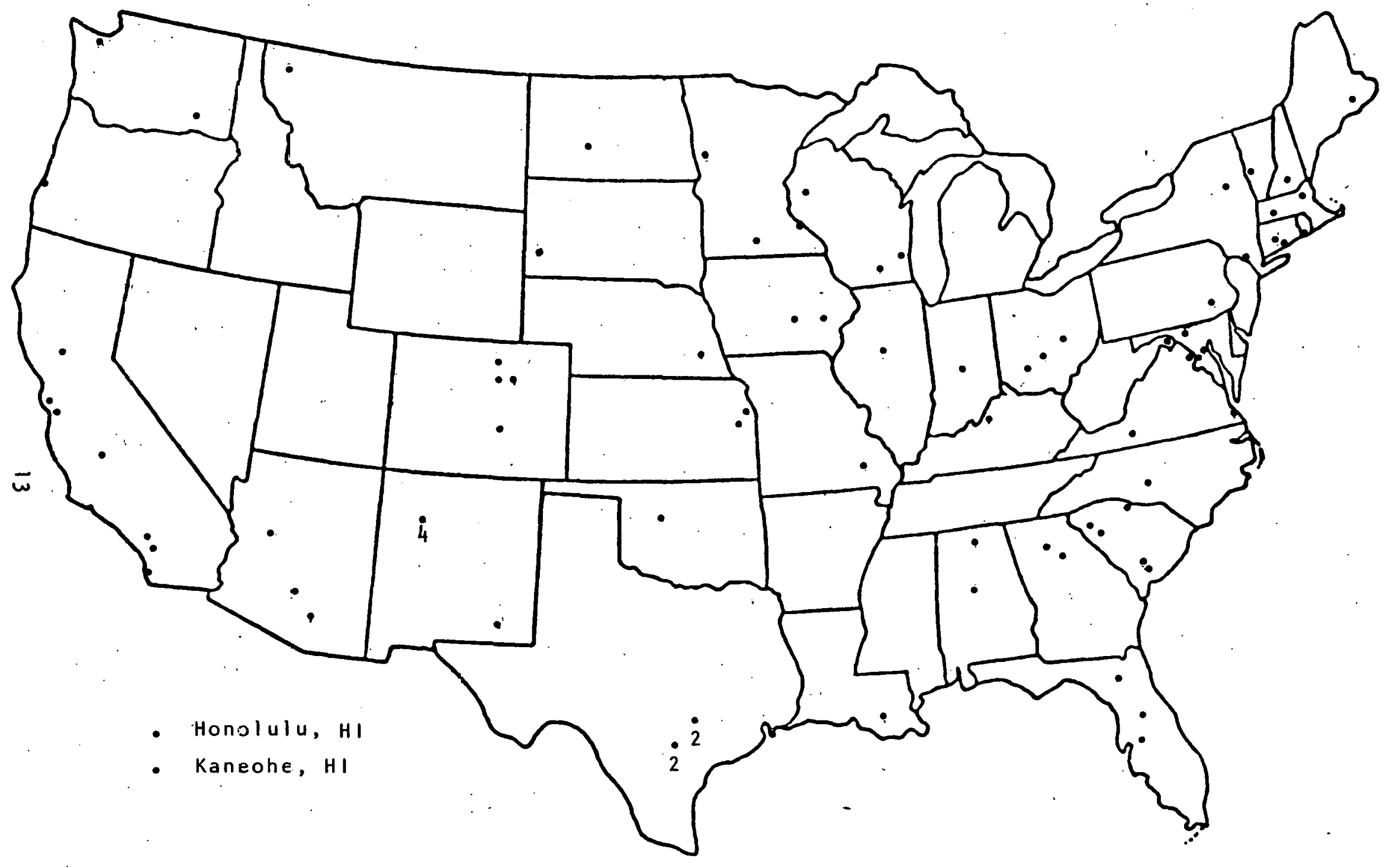

FIGURE 6 - LOCATION OF NATIONAL SOLAR HEATING AND COOLING DEMONSTRATION SITES 
APPENDIX A

SOLAR RADIATION OBSERVING STATIONS

WITH DATA ARCHIVED AT THE

NATIONAL CLIMATIC CENTER, ASHEVILLE, NORTH CAROLINA

Explanation:

- In January 1977 the National Weather Service

(NWS) revised its solar radiation data collection program. The stations continuing to collect data after January 1977 are 1 isted on page A-17. The NWS stations shown terminating during 1976 may have continued through December 1976. Specific stations may be verified by contacting the National Climatic Center, Asheville, North Carọlina.

- When two stations are listed in the same location, the hourly magnetic tape deck 280 may contain data from both stations.

- " $X$ " prefix in the Station Number indicates a cooperative station.

- Information about the rehabilitated solar radiation data may be found in SOLMET, Volume 2 Final Report, TD-9724, "Hourly Solar Radiation Surface Meteorological Observaliuns", Natiunal Climatic Center, Asheville, North Carolina.

- Solar radiation data from the new NWS network is published monthly by NCC at a subscription price of $\$ 8.40$ per year, domestic rate, entitled "Monthly Summary Solar Radiation Data". To date, data has been published through May 1977: The remaining past months are expected to be published soon. 


\begin{tabular}{|c|c|c|c|c|c|c|c|c|c|c|c|}
\hline $\begin{array}{l}\text { STATE } \\
\text { STATION } \\
\text { NUMBER } \\
\text { Elev, m } \\
\end{array}$ & $\begin{array}{l}\text { STATION } \\
\text { LOCATION } \\
\text { Lat/Long. Degrees }\end{array}$ & $\begin{array}{r}\text { TYPE } \\
\text { CHART }\end{array}$ & \multicolumn{2}{|c|}{$\frac{\text { HOUBLY DATA }}{\text { BEGIN END }}$} & \multicolumn{2}{|c|}{$\frac{\text { DAILE }}{\text { DEGIN }} \frac{\text { DATA }}{\text { END }}$} & REMARKS & \multicolumn{2}{|c|}{$\begin{array}{l}\text { TAPE DECK } 280 \\
\text { DOURLY DATA }\end{array}$} & $\begin{array}{l}\text { TAPE DECK } 480 \\
\text { DAILY DATA }\end{array}$ & NOTE \\
\hline $\begin{array}{l}\text { ALASKA } \\
25308 \\
34 \\
27502 \\
16 \\
26615 \\
49 \\
26411 \\
138 \\
\times 5733 \\
46 \\
\times 6870 \\
69\end{array}$ & $\begin{array}{l}: \\
\text { ANNETTE ISLAND } \\
55.03 / 131.57 \\
\text { BARROW } \\
71.3 / 156.78 \\
\text { BETHEL } \\
60.78 / 161.8 \\
\text { FAIRAANKS } \\
64.8 / 147.87 \\
\text { MATANUSKA } \\
61.57 / 149.27 \\
\text { PALMER } \\
61.57 / 149.1\end{array}$ & $\begin{array}{l}\text { CIR. } \\
\text { ROLL } \\
\text { ROLL } \\
\text { ROLL } \\
\text { ROLL }\end{array}$ & $\begin{array}{r}4 / 51 \\
1 / 50 \\
8 / 31 \\
11 / 55\end{array}$ & $\begin{array}{r}9 / 74 \\
11 / 75 \\
7 / 76 \\
7 / 76\end{array}$ & $7 / 49$ & 7/75. & $\begin{array}{l}\text { ROLL BEGAN } 10 / 49 \\
\text { ROLL BEGAN } 1 / 42 \\
\text { ROLL BEGAN } 4 / 54 \\
\text { TOTAL NET } \\
\text { EXCHANGE } \\
6 / 72-12 / 75\end{array}$ & $\begin{array}{l}1.57 \\
1.57 \\
1.57 \\
1.57\end{array}$ & $\begin{array}{l}3 / 57 \\
3 / 57 \\
3 / 57 \\
3 / 57\end{array}$ & $\begin{array}{cc}7 / 52 & 7 / 75 \\
7 / 52 & 10 / 74 \\
7 / 52 & 10 / 75 \\
7 / 52 & \text { TO DATE } \\
12 / 54 & \text { TO DATE } \\
1 / 67 & \text { TO DATE } \\
& \end{array}$ & $\begin{array}{l}\text { (B) } \\
\text { (B) } \\
\text { (B) } \\
\text { (C) }\end{array}$ \\
\hline$\frac{\text { ARKANSAS }}{\frac{13963}{84}}$ & $\begin{array}{l}\text { LITLEE ROCK } \\
34.73 / 92.23\end{array}$ & CIR. & $\begin{array}{l}7 / 49 \\
4 / 73\end{array}$ & $\begin{array}{l}3 / 52 \\
7 / 76\end{array}$ & & & $\begin{array}{l}\text { CIR. } 7 / 49-6 / 76 \\
\text { (FEW BREAKS) }\end{array}$ & & & $11 / 52$ TO DATE & \\
\hline
\end{tabular}

NOTE: (A) hOURLy datP. TABULATEd bi'T NOT ON MAG TAPE.

(B) ADDITIONAL HOURLY DATA EN PUNCH CARDS BUT NOT U.V MAG TAPE.

(C) CHARTS NRE IN THE NCC BRTT HOURLY DATA UNWORKED.

(D) HOURLY DATA DISCONTINUET IN 1967. CIIARTS CAN BE WORKED. 


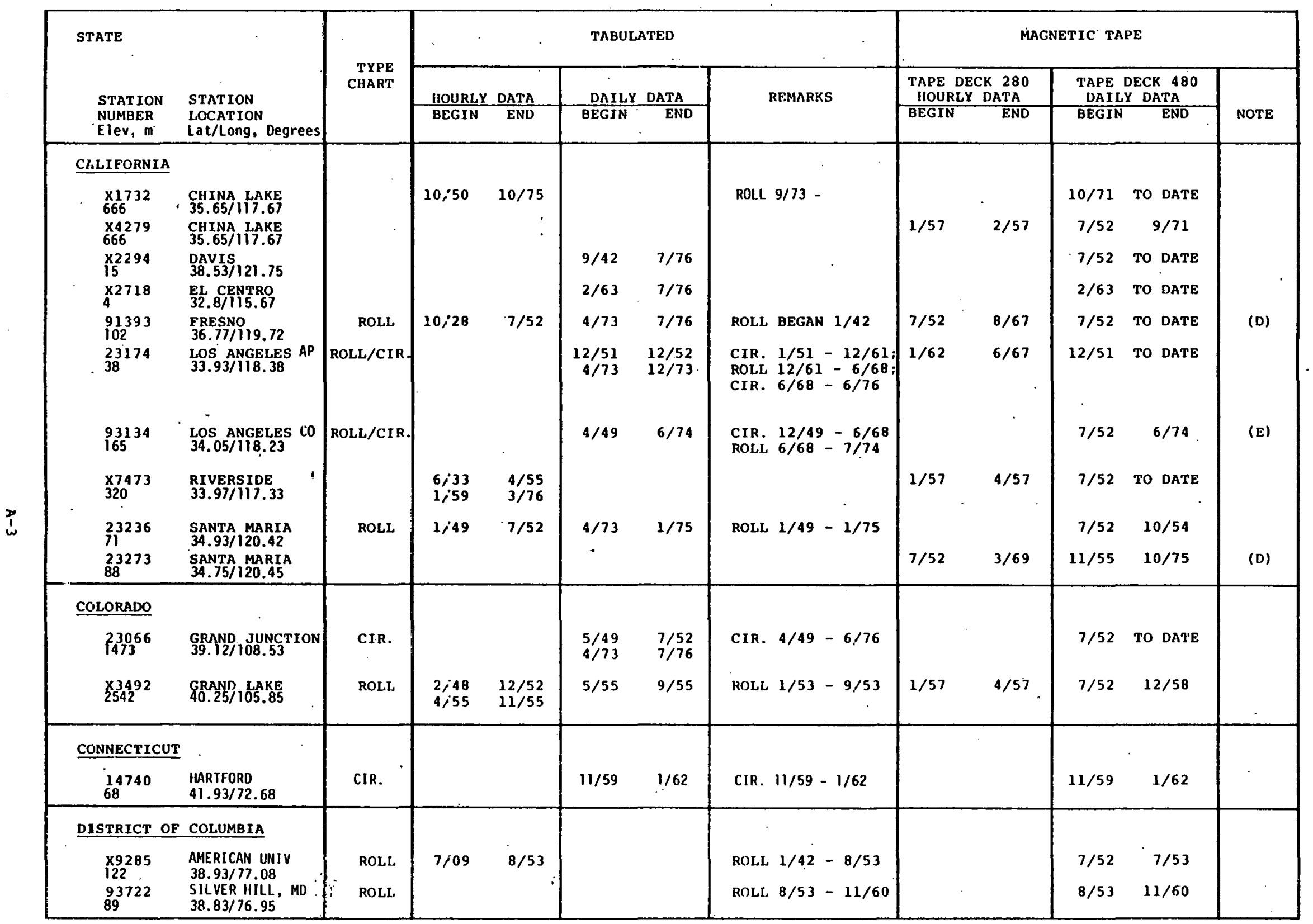

NCTE: (D) HOURLY DATA DISCONTINUED IN 1967. CHARTS CAN BE WORKED.

(E) HOURLY DATA UNWORKED AND CIIART'S ARE CONSIDERED UNRELIABLE. 


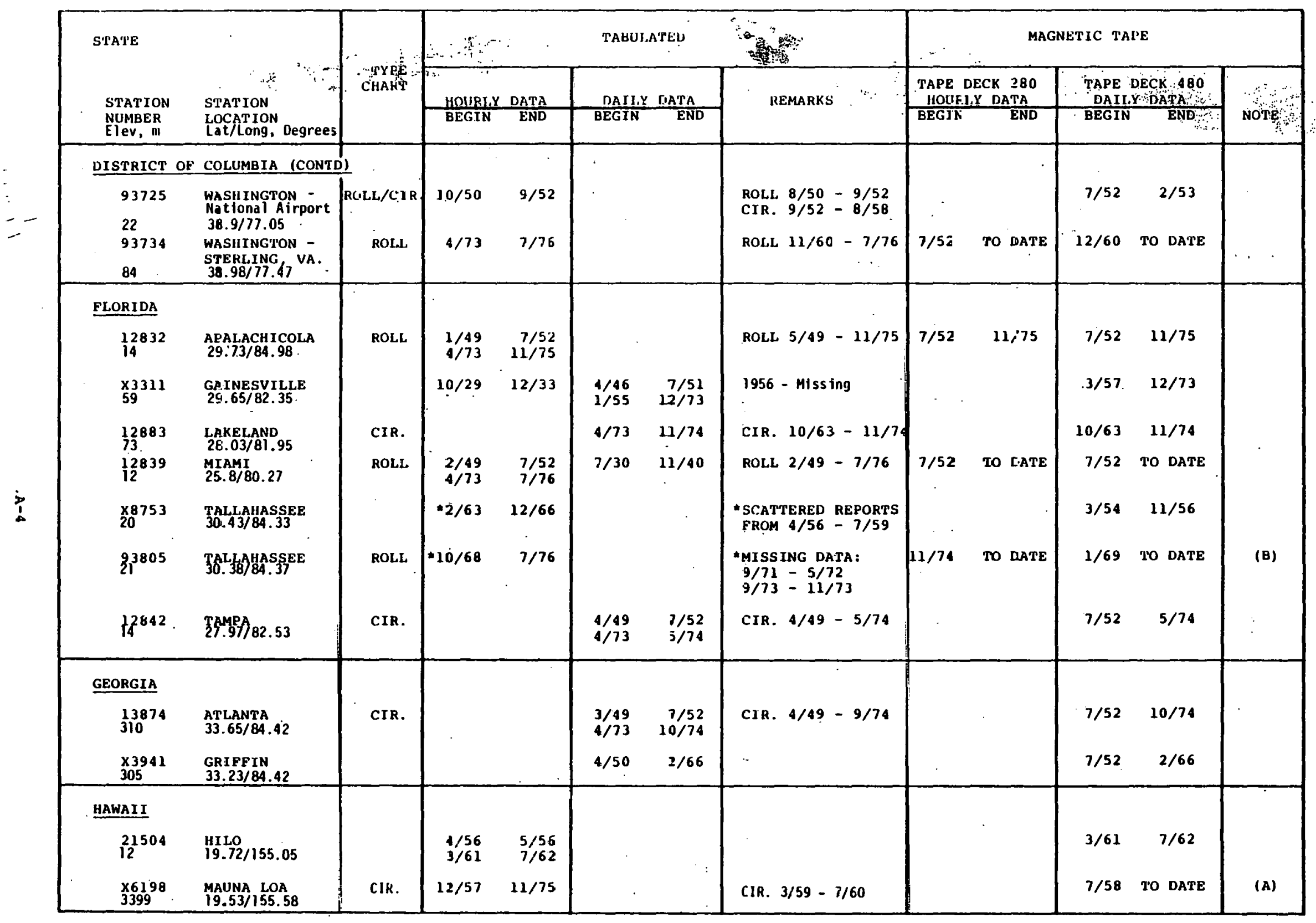

NOTE: (A) hOURLY DATA TABULATED But DOT ON MAG TAPE.

(B) additional houfly data on punch cards but not on mag tape. 


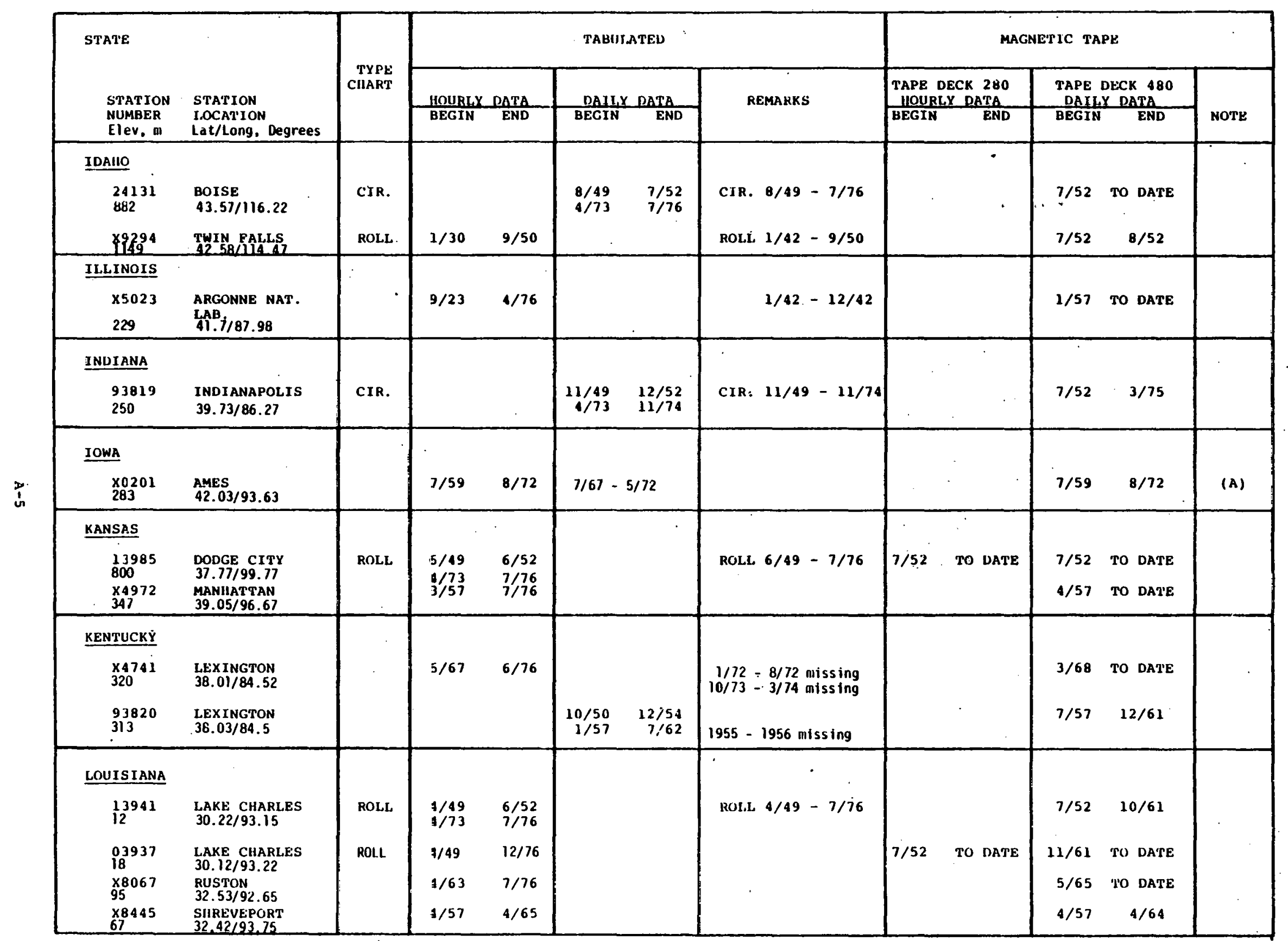

nOte: (A) hourly data tabulated but not on mag tape. 


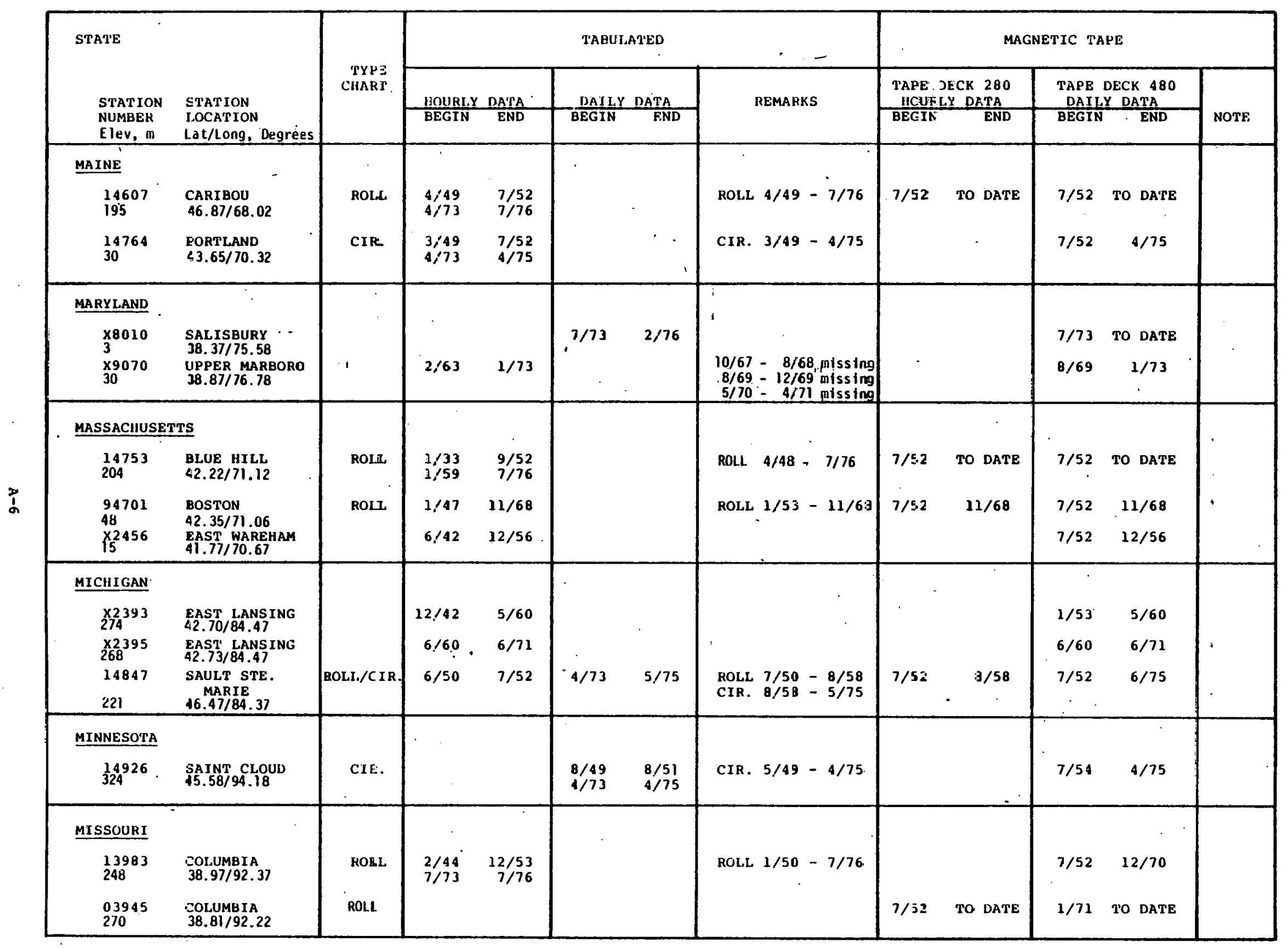




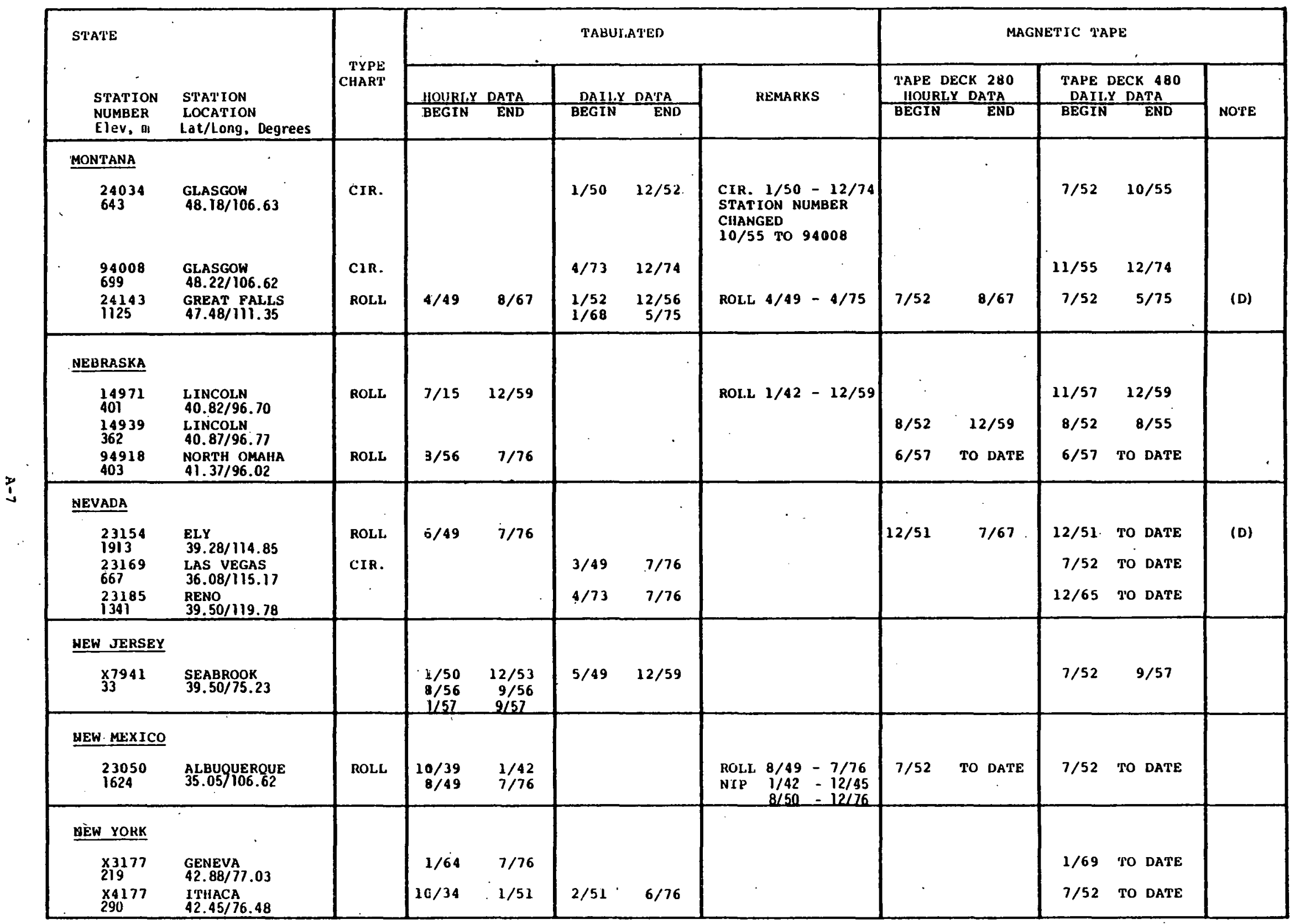

NOTE: (D) HOURLY DATA DISCONTINUEd IM 1967. CHARTS CAN BE WOIKED. 


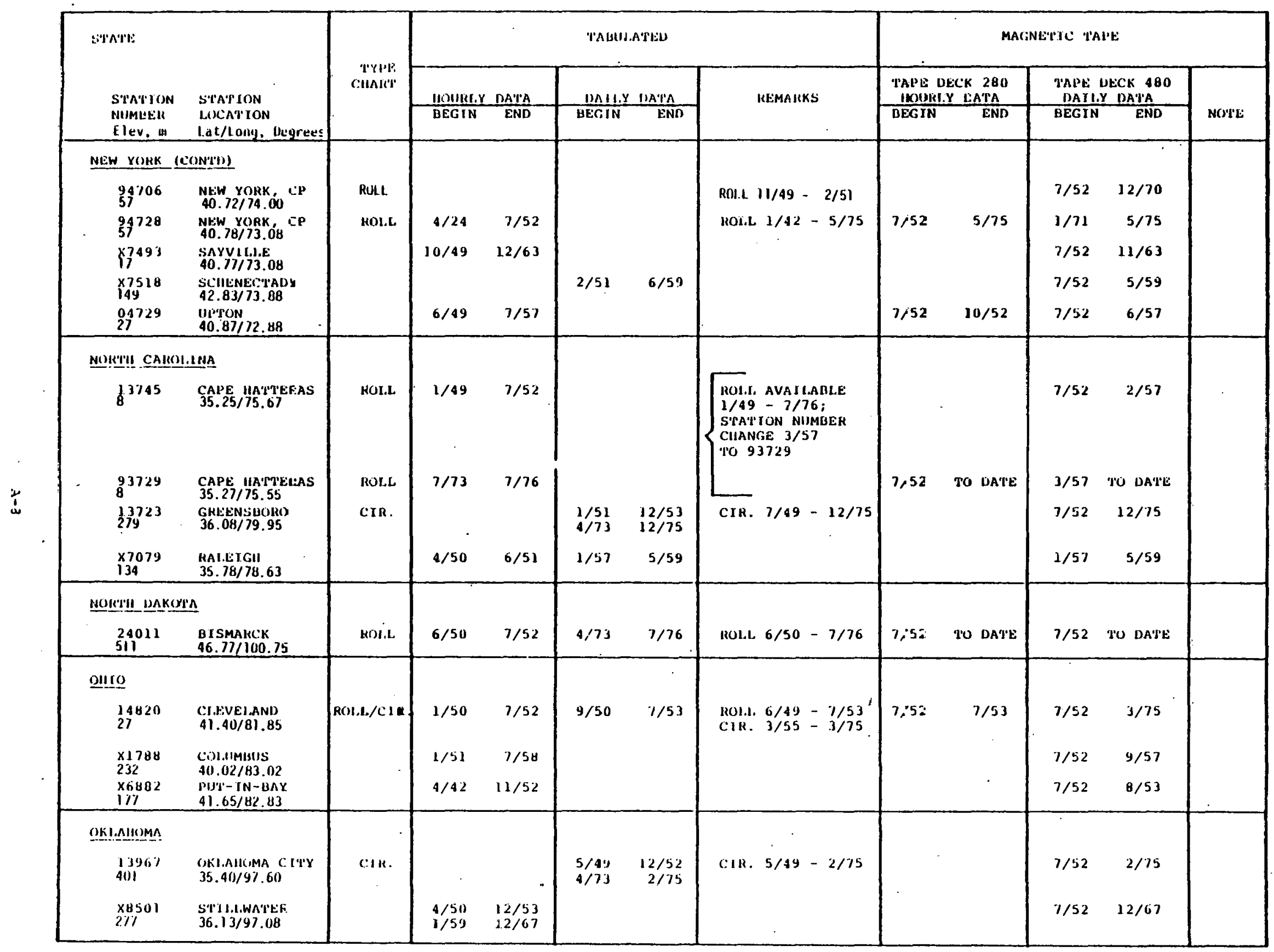




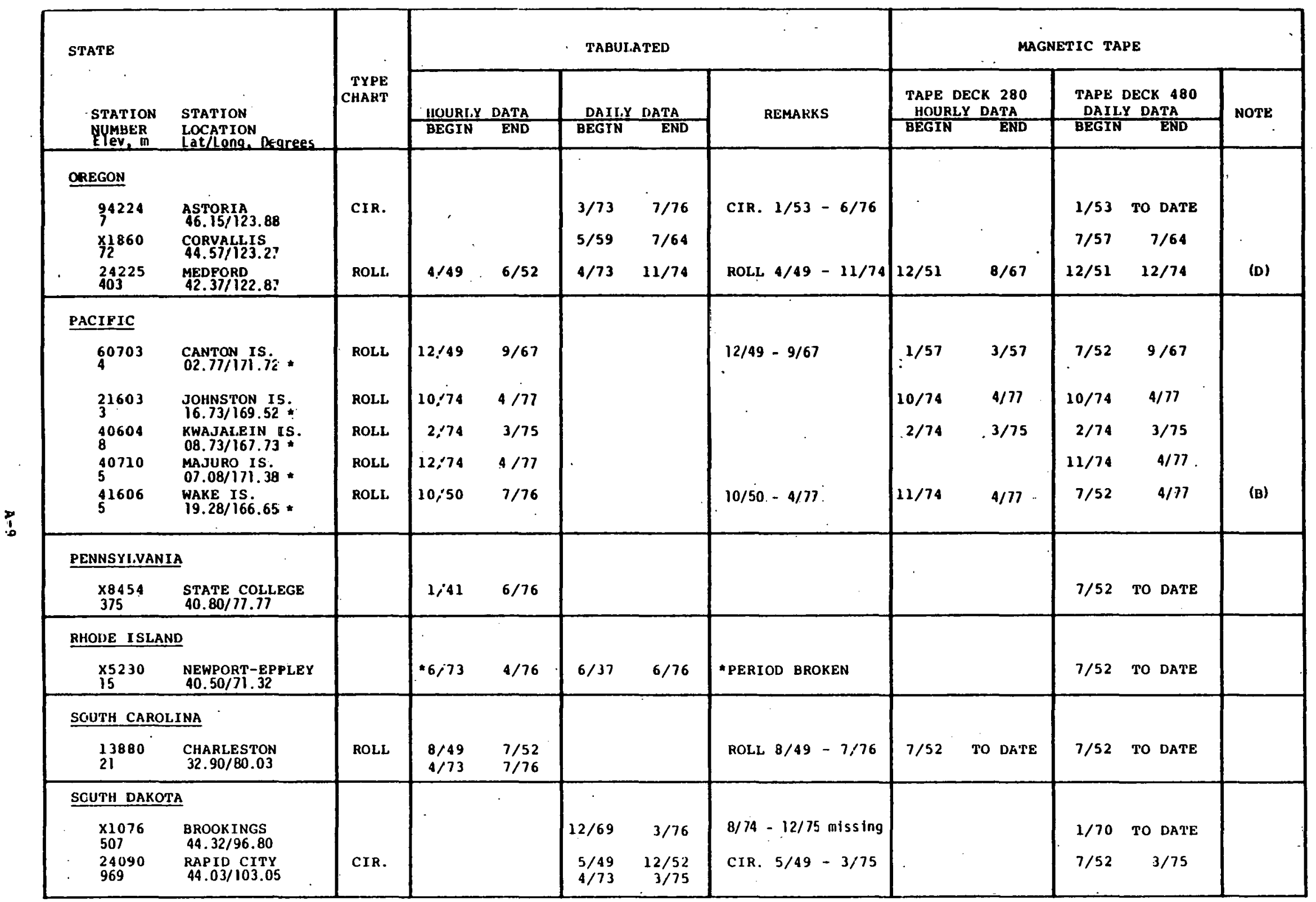

NOTE: (B) ADDITIONAL HOURLY DAT'A ON PUNCH CARDS BUT NOT ON MAG T'APE.

* note these are all. S. lat \& e. long

(D) hOURLY DATA DISCONTINUED IN 1967. CHARTS CAN BE WORKED. 


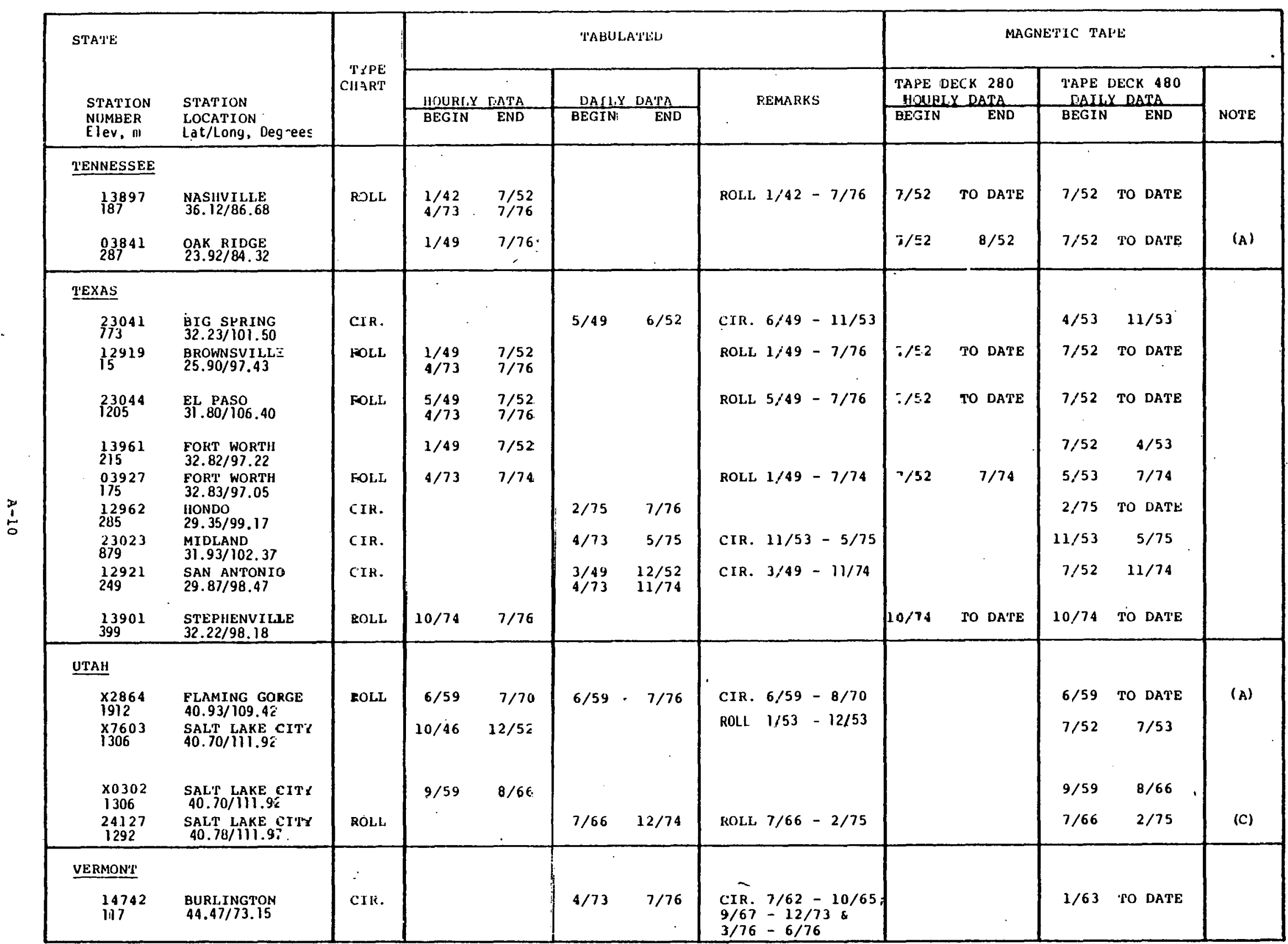

NOTE: (a) hourLy data Tabulated bu'P NO'T ON MAG TAPE.

(C) CHARTS ARI IN THE NCC BUT HOURLY DATA JHWORKEL. 


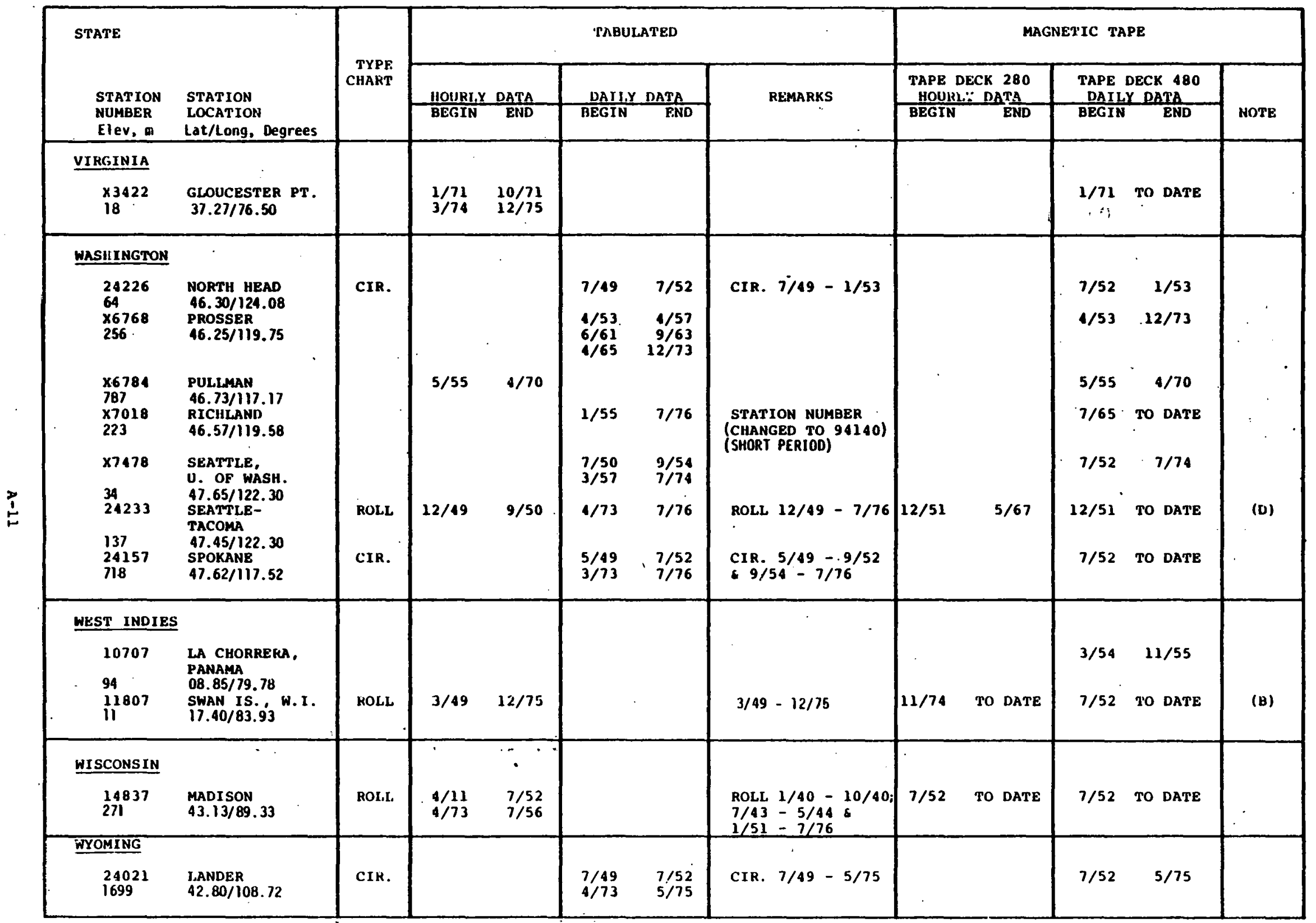

NOTE: (B) ADDJTYONAL hOUKLY DAT'A ON PUNCII CARDS BU'Y NOT ON MAG TAAPE.

(D) IOURLY DATA DISCOHTINIED IN 1967. CHARTS CAR BE WORKED. 


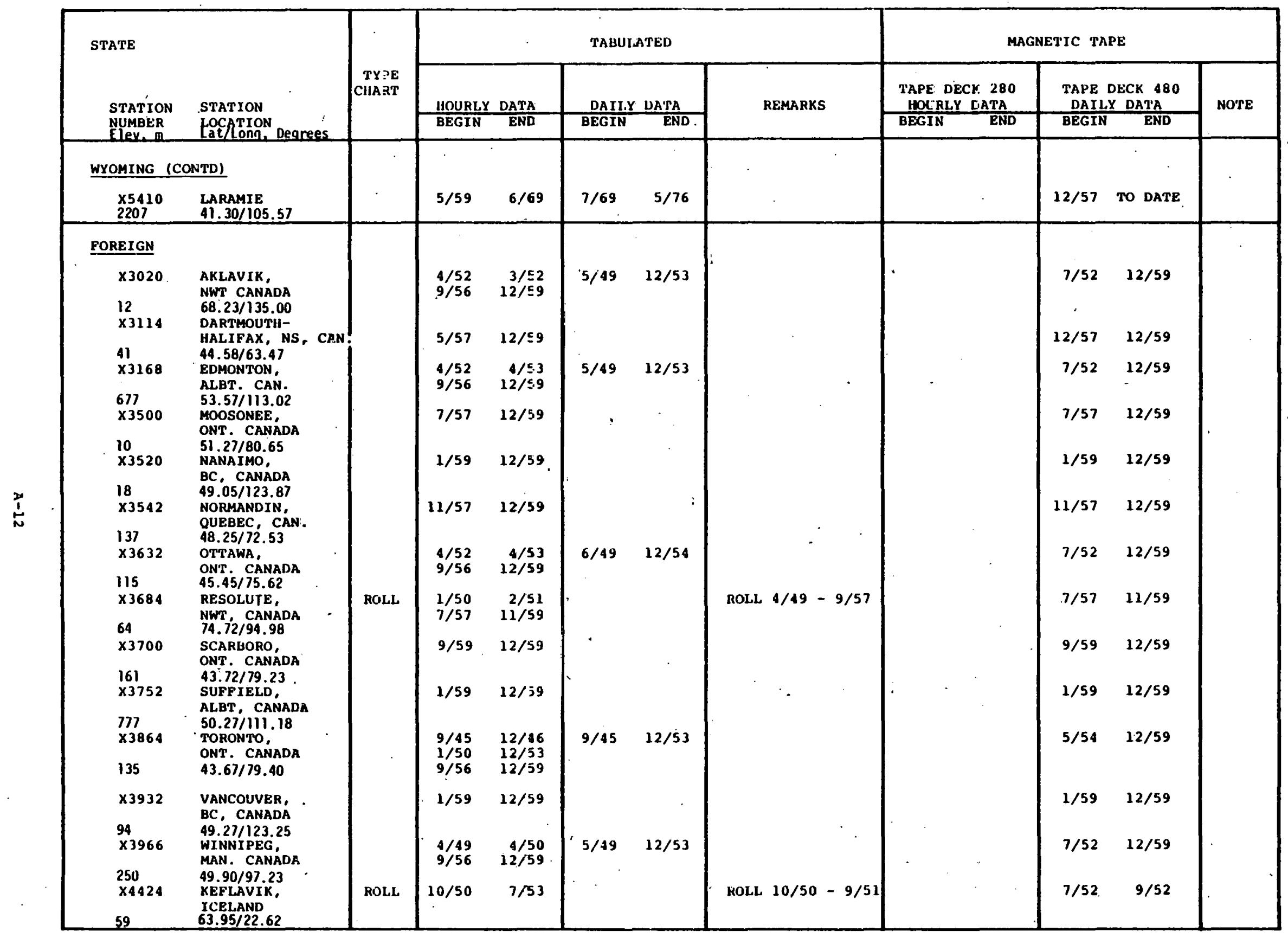


TYPICAL METEOROLOGICAL MONTHS COMPRISING

TYPICAL METEOROLOGICAL YEAR FOR 26 STATIONS

\section{Location}

Albuquerque, NM

Apalachicola, FL

Bi smark, ND

Boston, MA

Brownsville, TX

Cape Hatteras, NC

Caribou, ME

Charleston, SC

Columbia, Mo

Dodge City, KS

El Paso, TX

Ely, NV

Fort Worth, TX

Fresno, CA

Great Falls, MT

Lake Charles, LA.

Madison, WI

Medford, OR

Miami, FL

Nashville, TN

New York City; NY

North Omaha, NE

Phoenix, AZ

Santa Maria, CA

Seattle-Tacoma, WA

Waskington, DC

Jan Feb Mar

Apr

May

Jun

$58 \quad 53 \quad 6$

$\begin{array}{llll}60 & 60 & 64 & 6\end{array}$

6071

57

6653

6461

$65 \quad 55.56$

5970

70

6060

75

$65 \quad 55$

60

$74 \quad 67$

$74 \quad 71$

$\begin{array}{ll}72 & 61\end{array}$

$64 \cdot 75$

$\begin{array}{lll}68 & 65 & 71\end{array}$

$67 \quad 72$

56

6560

$\begin{array}{lll}66 & 62 \quad 53\end{array}$

$62 \quad 74$

67

$54 \quad 59$

$58 \quad 59$

$64 \quad 74$

6367

6469

Jul

Aug

Sep

Oct Nov Dec

6160

$54 \quad 57$

$67 \quad 63$

6358

$60 \quad 62$

65

6955

66

$67 \quad 65$

$59 \quad 54$

$56 \quad 6$

$65 \quad 71 \quad 64$

5356

$\begin{array}{llll}57 \quad 68 & 67 \quad 67\end{array}$

$56 \quad 62$

6257

6166

685

$\begin{array}{lll}57 & 61 & 61\end{array}$

6954.69

6968

$71 \quad 64$

6466

368.75

697

5374

$53 \quad 72$

73

$67 \quad 60$

606

6571

$74 \quad 66$

$\begin{array}{llll}54 & 70 \quad 58 & 64\end{array}$

62

63

$67 \quad 74$

7471

7460

$54 \quad 62 \quad 55$

$75^{\circ} 74 \quad 54$

$71 \quad 71 \quad 56$

563

61

60

66

547

6171

$67 \cdot 71 \quad 56$

5675

$58 \quad 73$

$\begin{array}{llll}66 & 66 & 63 & 65\end{array}$

6266

66

665

$65 \quad 55$

$55 \quad 5$

$\begin{array}{llll}57 & 68 & 71 & 62\end{array}$

16370

$59 \quad 54$

62

73

71

$71 \quad 65$

$\begin{array}{llll}6 & 74 & 64 & 59\end{array}$

6366

6475

$58 \quad 62$

$\begin{array}{lll}64 & 53 \quad 57 \quad 73\end{array}$

63

58

74

$65 \quad 54$.

$\begin{array}{lll}69 & 64 & 59\end{array}$

69

63

6260

$\begin{array}{lll}60 & 62 & 61\end{array}$

$\begin{array}{llll}9 & 64 & 53 & 57\end{array}$

63

57

$\begin{array}{lll}65 & 6168\end{array}$

$\begin{array}{llllllllllll}72 & 59 & 59 & 63 & 58 & 72 & 61 & 60 & 66 & 68 & 74 & 62\end{array}$

$\begin{array}{llllllllllll}68 & 75 & 63 & 57 & 68 & 56 & .74 & 72 & 72 & 68 & 59 & 66\end{array}$

$\begin{array}{llllllllllll}63 & 59 & 57 & 57 & 56 & 64 & 53 & 62 & 61 & 62 & 53 & 62\end{array}$

$\begin{array}{llllllllllll}75 & 71 & 62 & 72 & 61 & 59 & 62 & 70 & 62 & 66 & 68 & 56\end{array}$

$\begin{array}{llllllllllll}65 & 70 & 64 & 67 & 56 & 68 & 73 & 65 & 73 & 75 & 73 & 61\end{array}$

Typical Meteorological Years (TMY's) for the 26 stations, consisting of specific calendar months most representative, or typical, for the station and month, are available from the NCC on magnetic tape, formatted similar to SOLMET. SOLMET is a common tape format that is designed to provide, in a single fortran compatible tape, quality controlled hourly solar radiation and collateral meteorological data. The format is a metric conversion of all parameters currently available. SOLMET was designed to provide the solar energy users with easy access to all appropriate historical meteorological data that are normally available in digitized form.

For additional information on data available from the NCC see SOLMET, 1978, Volume I - "User's Manual", which can be obtained from the Director, National Climatic Center, Asheville, North Carolina, 28801. 
STATIONS WITH DERIVED GLOBAL

SOLAR RADIATION VALUES FROM THE CORRECTED MEASUREMENTS

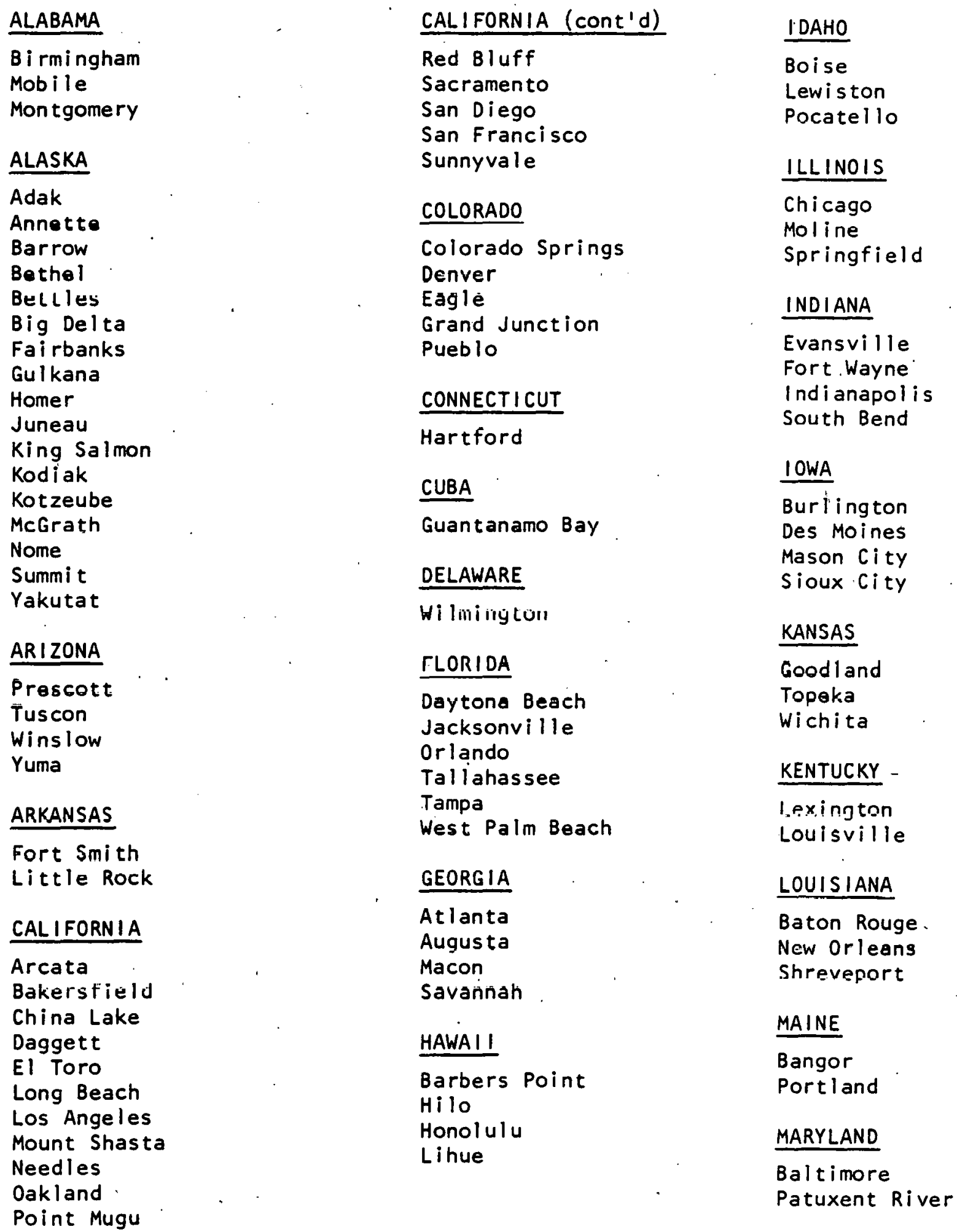




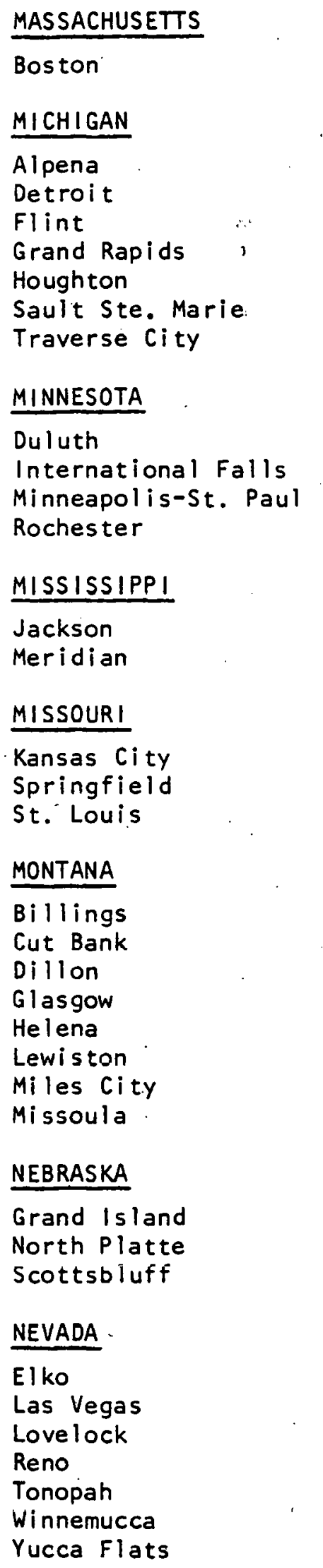

NEW HAMPSHIRE

Concord

NEW JERSEY

Lakehurst

Newark

NEW MEXICO

Clayton

Farmington

Roswell

Truth or Consequences

Tucumcari

Zun i

NEW YORK

Albany

Binghamton

Buffalo

Massena

New York City

Rochester

Syracuse

NORTH CAROLINA

Asheville

Charlotte

Cherry Point

Greensboro

Raleigh-Durham

NORTH DAKOTA

Fargo

Minot

$\mathrm{OH} 10$

Akron-Canton

Cincinatti

Cleveland

Columbus

Dayton

Toledo

Youngs town

OKLAHOMA

Oklahoma City

Tulsa
OREGON

Astoria

Burns

North Bend

Pendleton

Portland

Redmond

Salem

PACIFIC ISLANDS

Koror Island

Kwajalein I sland

Wake Island

PENNESYLVAN IA

Allentown

Erie

Harrisburg

Philadelphia

Pittsburgh

Wilkes-Barre-Scranton

PUERTO RICA

San Juan

RHODE ISLAND

Providence

SOUTH CAROLINA

Col umb i a

Greenville-Spartanburg

SOUTH DAKOTA

Huron

Pierre

Rapid City

Sioux Falls

TENNESSEE

Chat tanooga

Knoxville

Memphis

TEXAS

Abilene

Amarillo 


\begin{tabular}{lll} 
TEXAS (cont'd) & UTAH & WEST VIRGINIA \\
\hline $\begin{array}{l}\text { Austin } \\
\text { Corpus Christi } \\
\text { Dallas }\end{array}$ & $\begin{array}{l}\text { Bryce Canyon } \\
\text { Cedar City }\end{array}$ & $\begin{array}{l}\text { Charleston } \\
\text { Hel Riontington }\end{array}$ \\
Houston & Salt Lake City \\
Kingsville & VERMONT & WISCONSIN \\
Laredo & Burlington & Eau Claire \\
Lubbock & VIRGINIA & Green Bay \\
Lufkin & Norfolk & La Crosse \\
Midland-odessa & Richmond & WYoming \\
Port Arthur & Roanoke & Casper \\
San Angelo & WASHINGTON & Chyenne \\
San Antonio & Rock Springs \\
Sherilan & Olympia & Sheridan \\
Waco & Spokano & \\
Wichita Falls & Whidbey Island & \\
& Yakima &
\end{tabular}

The derived values from the 222 stations are available from the NCC in tape format, fortran compatible, containing both alphanumeric and numeric fields, see Input Data For Solar Systems, 1978. The retiabilitated data from the 26 stations listed in Appendix A-1.3.are available, also, in this tape format. 


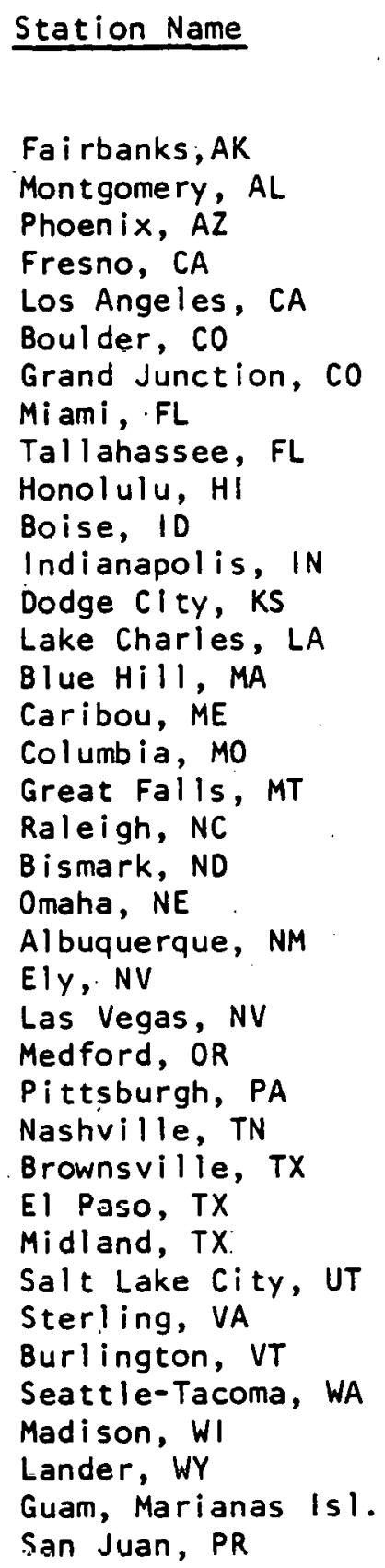

\begin{tabular}{l} 
Lat. (N) \\
(Degre $)$ \\
\hline \\
\\
64.82 \\
32.30 \\
33.43 \\
36.77 \\
33.93 \\
40.10 \\
39.12 \\
25.82 \\
30.38 \\
21.32 \\
43.57 \\
39.73 \\
37.77 \\
30.12 \\
42.22 \\
46.87 \\
38.82 \\
47.50 \\
35.87 \\
46.77 \\
41.37 \\
35.03 \\
39.28 \\
36.08 \\
42.37 \\
40.50 \\
36.12 \\
25.90 \\
31.80 \\
31.95 \\
40.77 \\
38.98 \\
44.47 \\
47.45 \\
43.13 \\
42.82 \\
13.45 \\
18.43 \\
\end{tabular}

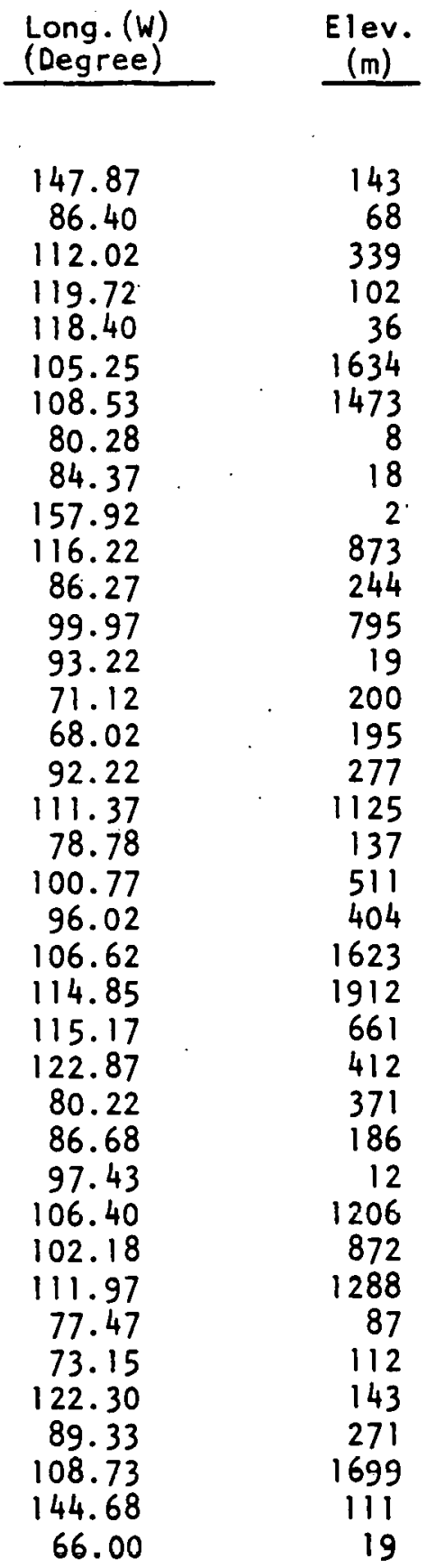


APPENDIX B

SOLAR RADIATION STATIONS

NOT IN THE BASIC NATIONAL WEATHER

SERVICE, NOAA, NETWORK

EXPLANATION OF SYMBOLS AND ABBREYIATIONS

ARS

ASFC

ATDL

BAAPC

D

DCWR

DOC

DOE

DNRC

DSE

DST

DWR

EDIS

EPA

ERAB

ERDA

ERL

FE

GMCC

HAS

HNE]

HSPA

IEA

JEEC

JPL

LBL

$M$

N

NCC

NOAA

NRL

NTIS

NU

NWS

NYSDEC
Agriculture Research Service

Atmosphefic Sciences Research Center

Atmospheric Turbulence and Diffusion Laboratory

Bay Area Air Pollution Control Service

Diffuse Radiation Measurement

DOUglas County Water Resources

Department of Commerce

DEPARTMENT OF ENERGY

Department of Natural Resources \& Conservation

Division of SOLAR ENERGY

Division of SOlar TECHNOLOGY

DEPARTMENT OF WATER Resources

EnVironmental Dáta and Information SERVICE

Environmental Protection Agency

Environmental Resources and Assessments Branch

ENERgY RESEARCH AND DEVELOPMENT ADMINISTRATION

ENVIRONMENTAL RESEARCH LABORATORY

FOWLKES ENGINEERING

Geophysical Monitoring gor Climatic Change

Harza Agricultural Services

Hawali Natural Energy Institute

Hawaitan Sugar Planters.' Association

INTERNATIONAL ENERGY AGENCY

JoHnson EnVI-Ronmental and EnERgy CEnTER

jet Propulsion lab

LAWRENCE BERKLEY LABS

ELEVATION ABOVE MEAN SEA LEVEL OF RADIOMETER IN WHOLE METERS

Latitude North of Radiometer.

National Climatic Center

National Oceanic and Atmospheric administration

National Radiation Laboratory

National TeChnical Information Service

NORTHEAST UTILITIES

National Weather Service

New York State Department of Environmental Conservation 
Period of Record

PGE
PG\&E
PPL
PSCC
RADIOMETER (RECORDER)
RI
RTI
S
SDGEC
SCE
SEHRTS
SERI
SIMM
SRL
T
TMSC
TVA
TYPE OF MEASUREMENT

UAH

UNIV

USA

USACE

USARS

USBR

USDA

USFS

USGS

$W$

WEST

()

( )
THE BEgINNING AND ENDING OF OBSERVATIONS ARE GIVEN, IF NO ENDING IS GIVEN, THE OBSERVATIONS WERE BEING Made as of December 31, 1978.

Portland General Electric

Pacific Gas and Electric Company

Pacific Power and light Company

Public Service Company of CO

THE RADIOMETER (RECORDER) LISTED̃ IS THE RADIOMETER (RECORDER) LAST USED AND OTHER TYPES COULD HAVE BEEN USED PREVIOUSLY, ROCKWELL INTERNATIONAL-

Research Triangle INSTItTUTE

Spectral Radiation Measurement

San Diego Gas and Electric Company

SOUTHERn California EDISON

Solar Energy Meteorological Research and Training Sites

SOLAR ENERGY RESEARCH INSTITUTE

Solar Insolation ileasurement in Montana.

SMITHSONIAN RadiATION LABORATORY

RADIOMETER NOT HORIZONTAL (TILTED)

Talcott Mountain Science Center

TENNESSEE VALLEY AUTHORITY

IF A PYRANOMETER OR PYRHELIOMETER IS' NOT FOLLOWED BY A LETTER

INDICAIING TILT, DIFFUSED OR SPECTRAL, THE INSTRUMENT IS

MEASURING GLOBAL OR DIRECT SOLAR RADIATION.

UnIVERSITY OF ALABAMA IN HUMTSVILLE

UNIVERSITY

UNITED STATES ARMY

US ARMY Corp of ENoINEERS

US Agricultural Research Service

US BUREAU OF RECLAMATION

US Department of Agriculture

US FoREst SERVICE

US. GEOLOGICAL SURVEY

LONGITUDE HEST OF RADIOMETER

Western ENergy Supply \& Transmission Associates

NUMBER IN PARENTHESES AFTER A RADIOMETER INDICATES NUMBER IN USE NUMBER IN PARENTHESES IN THE RECORDER - RECORD FORM COLUMN INDICATES DATA IN A PUBLICATION LISTED IN APPENDIX $C$ 
APPENDIX B

(CONTD)

STATION LOCATION

\section{RADIOMETER}

TYPE, MFG. MODEL
RECORDER

RECORD FORM
PERIOD OF RECORD

ALABAMA

Pyranometer, Eppley

10-JUNCTION

Printed tape

T́abulated (2)

1964 -

N $32^{\circ} 15^{\prime}$

W $85^{\circ} 30^{\prime}$

$199 \mathrm{M}$

Auburn Agricultural

EXPERIMENT StATION

Decatur

N $34^{\circ} 42^{\prime}$

W $87^{\circ} 06^{\prime}$

$182 \mathrm{M}$

TVA

FLORENCE
N $34^{\circ} 58^{\prime}$
W $88^{\circ} 13^{\prime}$
170 'M
TVA

HUNTSVILLE

N $34^{\circ} 44^{\prime}$

W $86^{\circ} 40^{\prime}$

$201 M$

UAH

Huntsville

N $34^{\circ} 39^{\prime}$

W $85^{\circ} 45^{\prime}$

$191 \mathrm{M}$

LOCKHEED

Muscle Shoals

N $34^{\circ} 39^{\prime}$

W $87^{\circ} 46^{\prime}$

$165 \mathrm{M}$

TVA
Pyranometer, Eppley 8-48; PyRRadiometer, TeledYNe GEOTECH TCH - 188-01
Mag tape Nova 1200 1/78-

HeWlett Packard 345

Pyranometer, Eppley 8-48; Mag tape Nova 12001967 Pyrradiometer, Teledyne Hewlett Packard 345 GEOTECH TCH - 188-01

\author{
Pyranometers (4) (T) Mag tape - SCI, \\ $12 / 77-$ \\ EPPLEY 8-48 (2), PSP, \\ UNIVAC $1100 / 10$ \\ Weather Measure Stak; \\ PyrheLIOMETER, EPPLEY \\ NIP \\ Pyranometer, Hycal \\ STRIP CHARTS \\ $10 / 75-5 / 77$
}

Pyranometer, Eppley 8-48; itag tape - SCI, Pyrradiometer, Teledyne Univac 1100/10 FEOTECH TCH - 188-01 


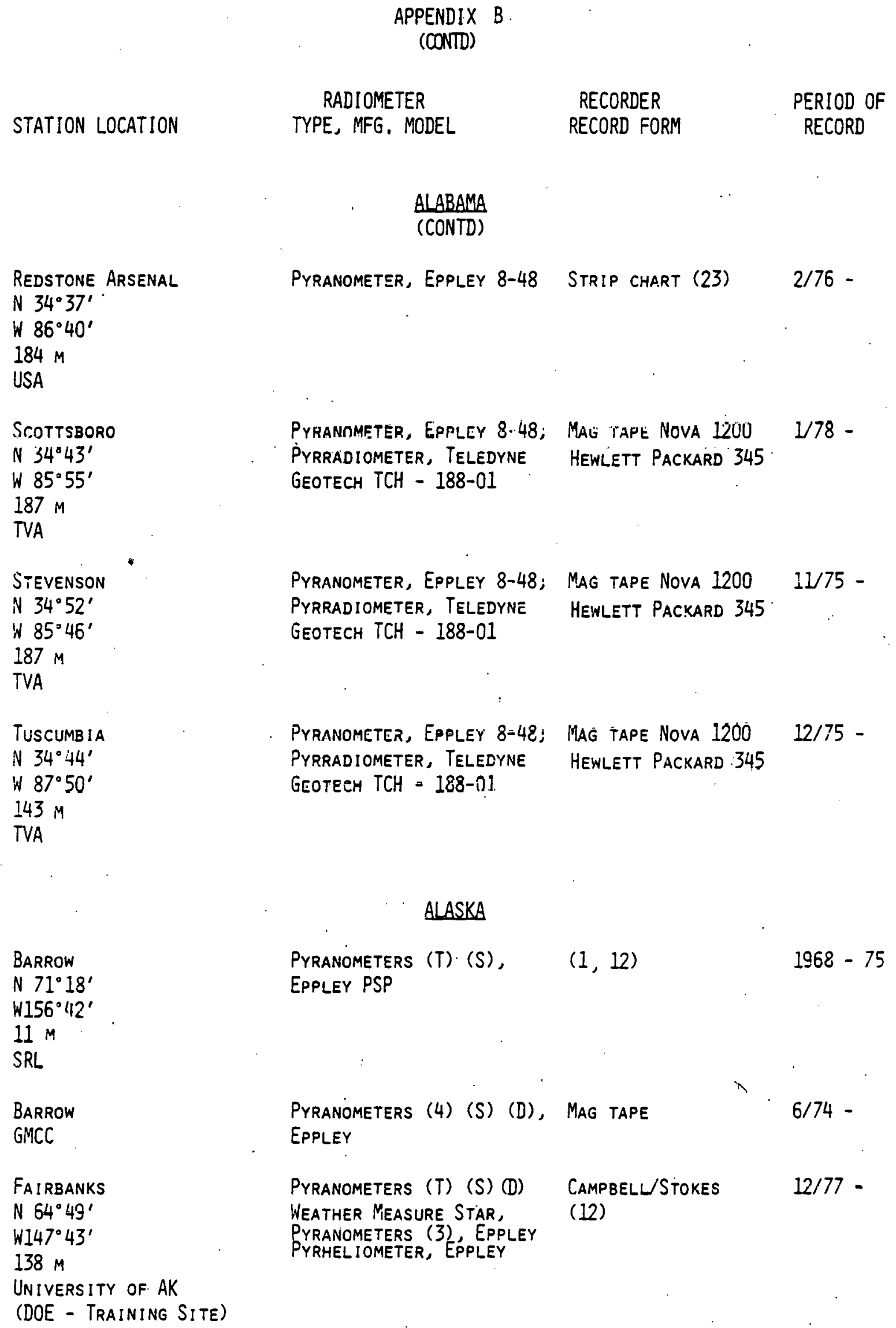

\section{ALABAMA}

(CONTD)

Redstone Arsenal

N $34^{\circ} 37^{\prime}$

W $86^{\circ} 40^{\circ}$

$184 \mathrm{M}$

USA

STOTTSBORO

N $34^{\circ} 43^{\prime}$

W $85^{\circ} 55^{\prime}$

$187 \mathrm{M}$

TVA

Pyranometer, Eppley 8-48 Strip chart (23) 2/76 -

Pyrannmeter, Eppley 8.48; Mati tape nova 1200 1/78Pyrradiometer, Teledyne Hewlett Packard 345 GEOTECH TCH - 188-01

STEVENSON

Pyranometer, Eppley 8-48; Mag tape Nova 1200 11/75 -

N $34^{\circ} 52^{\prime}$

W $85^{\circ} 46^{\prime}$

$187 \mathrm{M}$

TVA

TUSCUMBIA

N $34^{\circ} 44^{\prime}$

W $87^{\circ} 50^{\prime}$

$143 \mathrm{M}$

TVA

Pyrradiometer, Teledyme Hewlett Packard 345

GEOTECH TCH - 188-01

Pyranometer, Eppley 8-48; Mag tape Nova 1200 12/75 Pyrradiometer, Teledyne Hewlett Packard 345 GEOTEEH TCH = 188-01.

ALASKA

Pyranometers (4) (S) (D), Mag tape

EPPLEY

PyRanometers (T) $(S)$, EPPLEY PSP

$(1,12)$

$1968-75$

Pyranometers (T) (S) (D) Weather Measure Star,

PYRANOMETERS (3), EPPLEY
PYRHELIOMETER, EPPLEY

CAMPbell/Stokes

$12 / 77$ -

(12) 
Castle Creek

Ni $33^{\circ} 43^{\prime}$

$W 109^{\circ} 11^{\prime}$

2444 M

USFS .

FLAGSTAFF

N $35^{\circ} 11^{\prime}$

W111.39'

$2112 \mathrm{M}$

UNIVERSITY OF AZ

Fort HUachuca

N $32^{\circ} 05^{\prime}$

$W 114^{\circ} 24^{\prime}$

$99 \mathrm{M}$

USA

Gila Beńd

N $32^{\circ} 56^{\prime}$

WI12 $43^{\prime}$

$224 \mathrm{M}$

Arizona Public Serivce

Lake Mojave

DEPT, of INTERIor

Mesn

N $33^{\circ} 20^{\circ}$

WI11 38'

$424 \mathrm{M}$

GM Proving Ground

Phoenix

N $33^{\circ} 27^{\prime}$

W112 $05^{\circ}$

$328 \mathrm{M}$

Arizona Public Service

Phoenix

N $33^{\circ} 50^{\prime}$

W112 $10^{\circ}$

$610 \mathrm{M}$

DESERT SUNSHINE EXPOSURE
RADIOMETER

TYPE, MFG. MODEL

\section{ARIZONA}

APPENDIX B

(CONTD)

- Pyranograph, Belfort

(3)

1966 -

RECORDER

RECORD FORM

PERIOD OF

RECORD

$1974-$

1975 -
Pyranometer, Eppley PSP Mag tape by 2/1977 Pyrheliometer, EPPLEY NIP SCE (17)

Pyranometer, Eppley

$1959-1961$

Pyranometer (T), Epplegy Strip chart

(3)

Pyranometer, Eppley PSP

MAG TAPE BY SCE

$(3,17)$

1966 -

3/1977 -

Pyranometer (2) (T) (D), Strip chart

EPPLEY PSP 8 50, SPECTRO- $(3,4)$

LAB SR-75; PyRheliometers,

EPPLEY NIP 
APPENDIX B

(CONTD)

STATION LOCATION

RADIOMETER

TYPE, MFG. MODEL

ARIZONA

(CONTD)

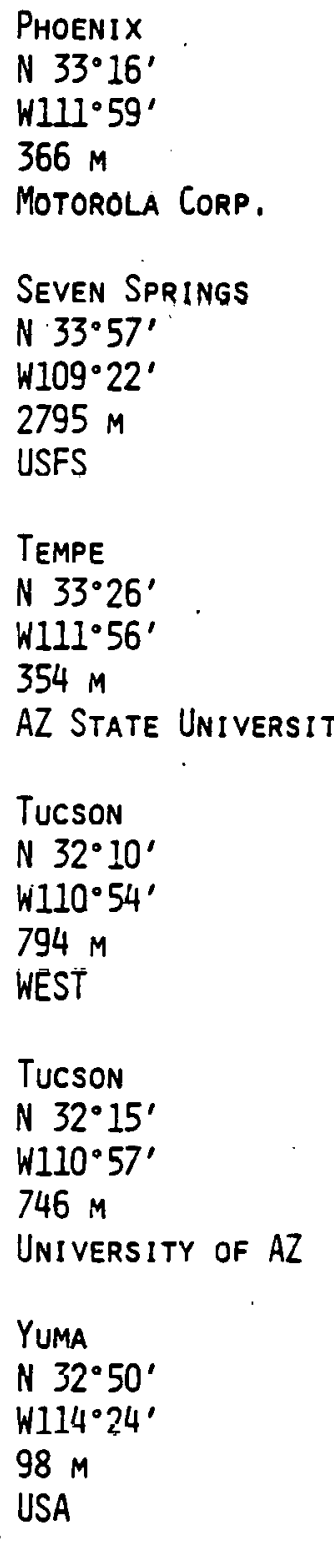

Phoenix

N $33^{\circ} 16^{\prime \prime}$

$W 111^{\circ} 59^{\circ}$

$366 \mathrm{M}$

SEVEn SPRINGS

N $33^{\circ} 57^{\circ}$

H $109^{\circ} 22^{\prime}$

$2795 \mathrm{M}$

TEMPE

N $33^{\circ} 26^{\prime}$

W $111^{\circ} 56^{\prime}$

354 M

AZ State University

Pyranometer

PYRHELIOMETER

Campbell/Stokes

$10 / 74-' 75$

Pyranograph, Belfort

(3)

1966 -

Pyranometer (T) (D)

PYRHELIOMETER

STRIP CHART

(3)

$1 / 77-$

Pyranometer, Eppley PSP

Mag tape by SCE

Pyrhel igmeter, Eppley NIP (17)

Pyranometers, (D)

PYRHELIOMETER

STRIP ChART

(3)

$1966-$

Pyranometer, Eppley

Strip CharT

1961 -

PyRHELIOMETER, EPPLEY

15-2 HONEYWELL

PERIOD OF

RECORD

1977 -

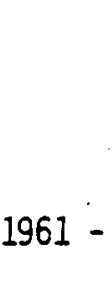

CONWAY

N $34^{\circ} 04^{\prime}$

W $92^{\circ} 27^{\prime}$

\footnotetext{
$\therefore \quad 91$ univ of Central aR

$\therefore \quad 91 \mathrm{M}$
}

\section{ARKANSAS} \\ Pyranometer, Weather \\ Measure Star \\ StRIP Chart \\ $6 / 78-$
}

B-6 
APPENDIX B

(CONTD)

STATION LOCATION

RADIOMETER

TYPE, MFG. MODEL

\section{CALIFORNIA}

Pyranometer, Eppley 8-48 Mag tape

ACTINOGRAPH

CF CASElla \& Co.

Pyranometer, Eppley 8-48 Mag tape

ACtinOGRAPY

N $35^{\circ} 14^{\prime}$

$W 118^{\circ} 52^{\prime}$

$133 \mathrm{M}$

DWR

\section{AUBURN}

N $38^{\circ} 54^{\prime}$

- W21.04'

394 in

PEE/LBL

BAKERSFIELD IOS

N $35^{\circ} 14^{\prime}$

$W 118^{\circ} 59^{\prime}$

$100 \mathrm{M}$

DWR

BARRETT RES

N $32^{\circ} 57^{\prime}$

$W 116^{\circ} 42^{\prime}$

$267 \mathrm{M}$

USGS
Pyranometer, Eppley 8-48 Mag tape

ACTINOGRAPH

$(5,28)$

$1969-1970$

Pyranometer, Eppley

LIGHTBULB TYPE, 10

THERMOPILE JUNCTION

$7 / 77-$

(5)

$1960-1961$
SCE $(5,16,17)$

(5)

$1958-1964$

(17)

(5)

$1959-1965$

PERIOD OF

BECORD

1975 -

$9 / 77-$ 
STATION LOCATION

\begin{abstract}
APPENDIX B
(CONTD)
\end{abstract}

RADIOMETER

TYPE, MFG. MODEL
RECORDER

RECORD FORM
PERIOD OF

RECORD

\title{
CALIFORNIA \\ (CONTD)
}

BARRETT RES

N $32^{\circ} 41^{\prime}$

(24)

W $116^{\circ} 40^{\prime}$

$495 \mathrm{M}$

$\mathrm{LBL}$

BARSTOW

N $34^{\circ} 53^{\prime}$

WI1 'UU'

$664 \mathrm{M}$

SCE

BERKELEY

N $37^{\circ} 52^{\prime}$

W $122^{\circ} 15^{\prime}$

105 M

PGE/LBL

BLYTHE

N $33^{\circ} 36^{\prime}$

W11 $4^{\circ} 36^{\prime}$

$80 \mathrm{M}$

SCE

BRAWLEY

N $32^{\circ} 57^{\prime}$

W $115^{\circ} 33^{\prime}$

$43 \mathrm{M}$

USARS

Berenda 2N

N $37^{\circ} 04^{\prime}$

$W 120^{\circ} 08^{\prime}$

$82 \mathrm{M}$

DWR

BURLINGAME

N $37^{\circ} 35^{\prime}$

W122 21'

- $8 \mathrm{M}$

BAAPC
Pyranometer (D), Eppley 8-48; PYRHEL IOMETER. EPPLEY NIP

Pyranometer, Eppley 8-48 Mag tape

$4 / 77-$

Pyranometer, Eppley $8-48 \quad(3,17)$

Pyrheliometer NIP

ASSUMEd TO BE THERMOPILE (5)

$1967-1969$

$(3,5,17)$

$1976-$ TYPE

ACTINOGRAPH

(5)

$1962-1963$

Pyranometer, Eppley 8-48

(5)

$1973-1976$ 


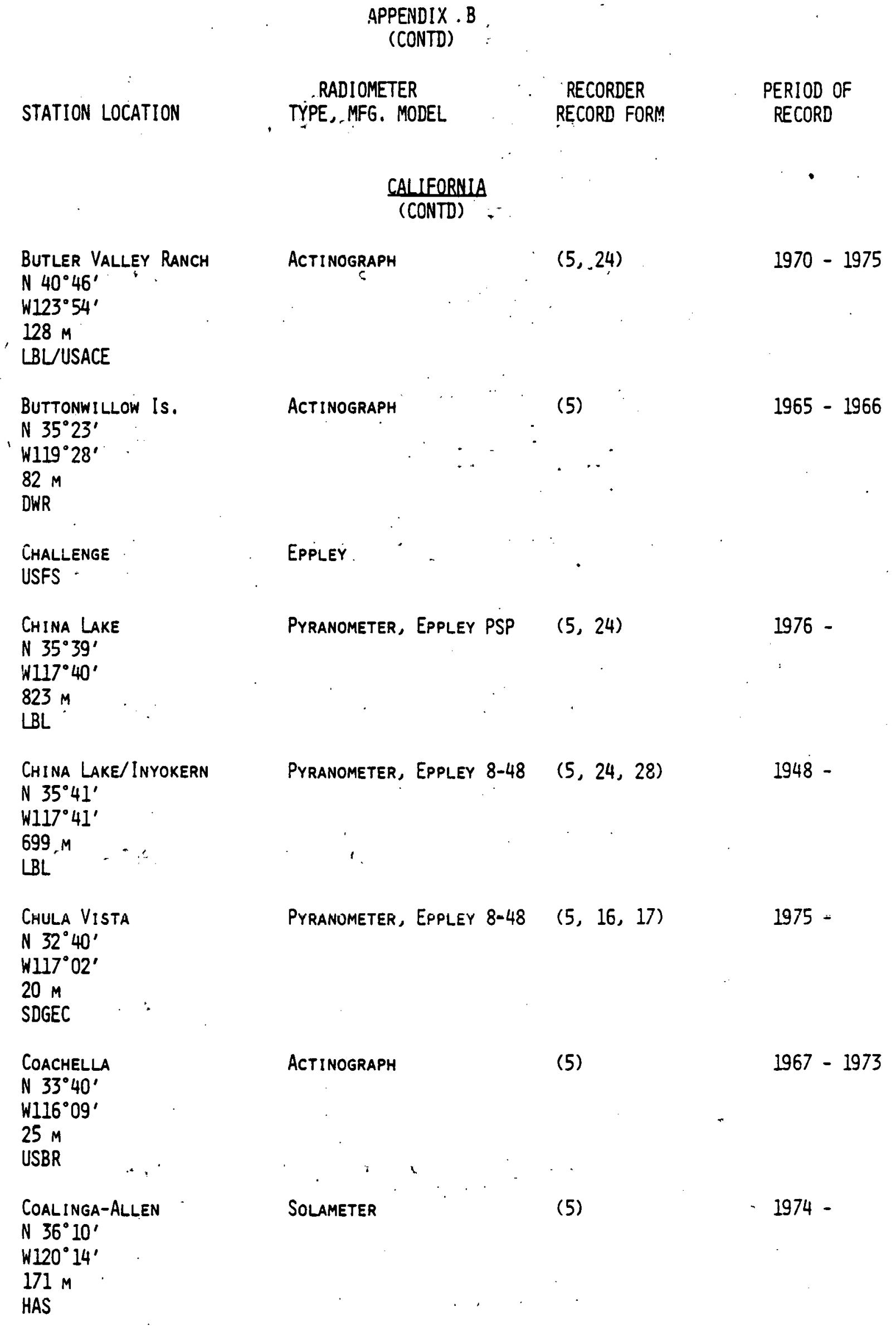




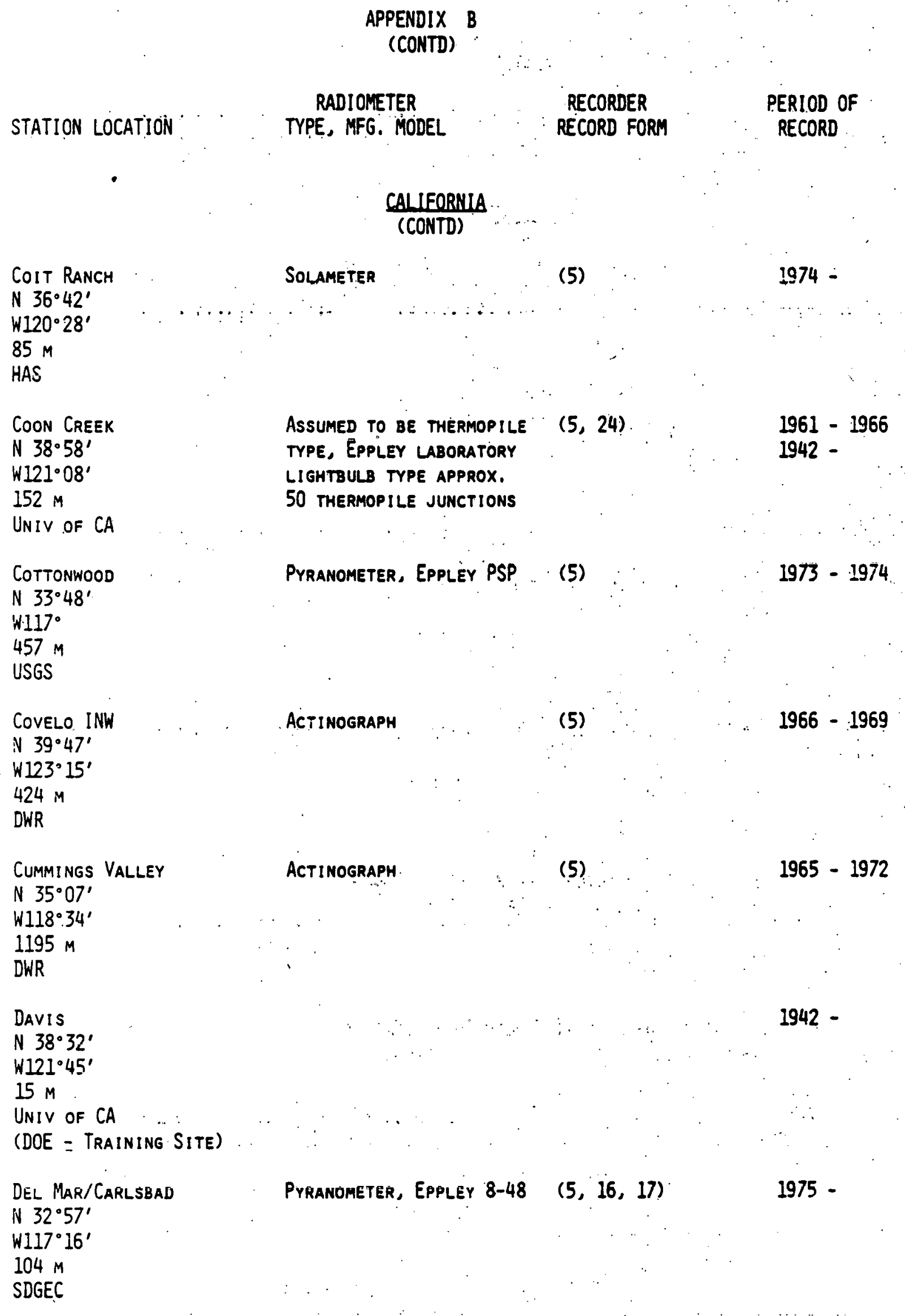


STATION LOCATION,

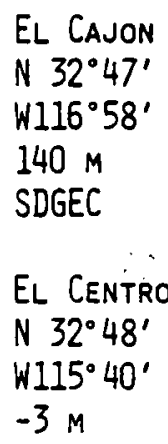

LBL/DWR/NWS

EL SEgundo

N $33^{\circ} 55^{\prime}$

$W 118^{\circ} 25^{\prime}$

$12 \mathrm{M}$

SCE

EL TORO

N $33^{\circ} 38^{\prime}$

$W 117^{\circ} 43^{\prime}$

$110 \mathrm{M}$

SCE

ESCONDIDO

$N 33^{\circ} 08^{\prime}$

W117 $06^{\circ}$

$216 \mathrm{M}$

SDGEC

EUREKA

N $40^{\circ} 48^{\circ}$

W $124^{\circ} 10^{\prime}$

$13 \mathrm{M}$

PGE/LBL

FInLEY 2SW

N $38^{\circ} 59^{\prime}$

$W 122^{\circ} 54^{\prime}$

$415 \mathrm{M}$

DWR
APPENDIX B

(CONTD)
RADIOMETER

TYPE, MFG. MODEL
RECORDER

RECORD FORM
RECORD
PERIOD OF
CALIFORNIA (CONTD)

Pyranometer, Eppley $8-48 \quad(5,16,17)$

1975 -
Pyranometer $\quad \therefore \quad \cdots \quad(3,5,24) \quad 1963-$

Pyranometer, Eppley $8-48$ " $(5,17)$ 1976 -
PYRHELIOMETER, EPPLEY NIP
Pyranometer, Eppley $8-48 \quad(5,16)^{\circ} .$.

Pyranometer, Eppley 8-48 Mag tape 7/78-

ACTINOGRAPH

(5)

$1972-1973$ 


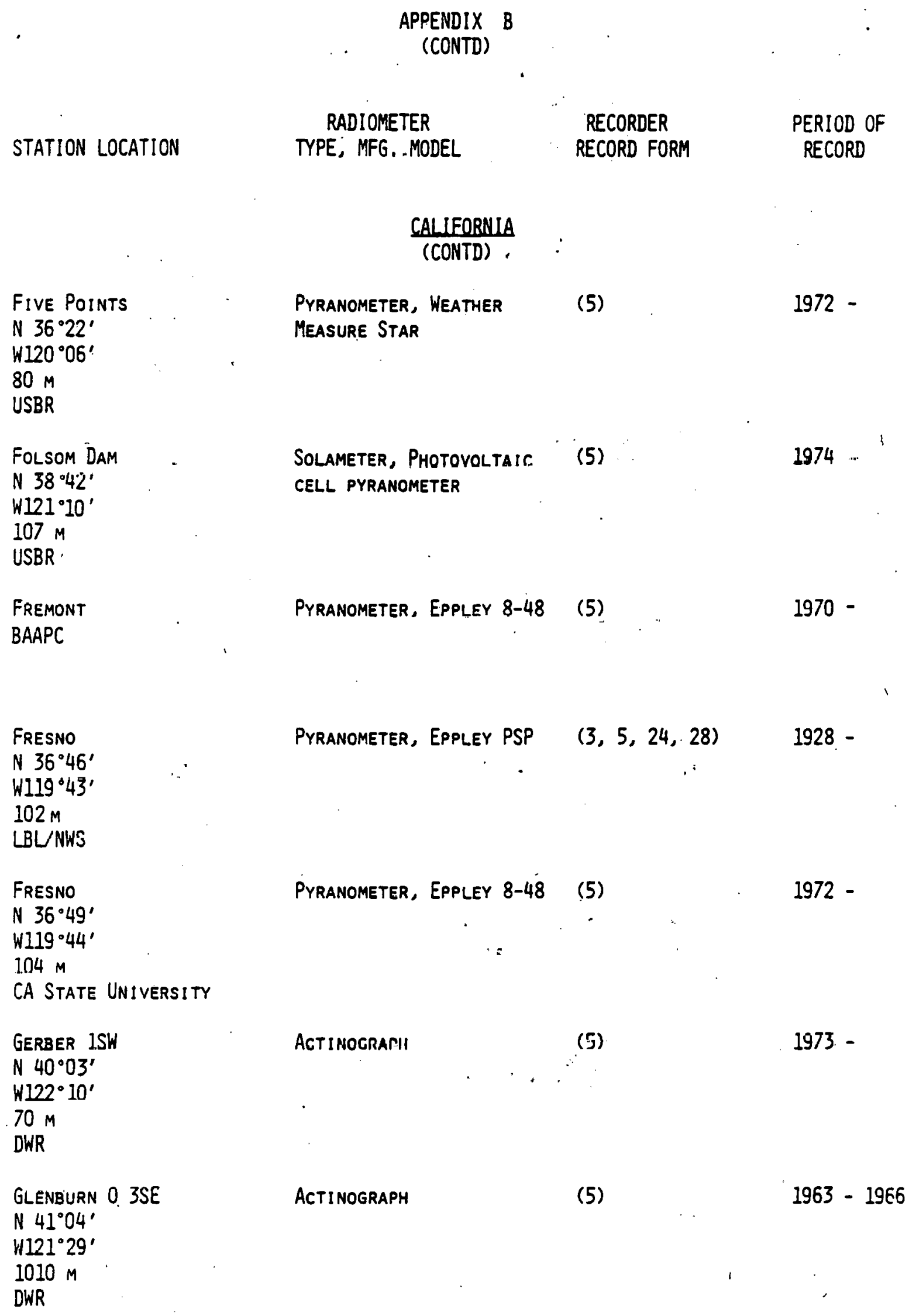




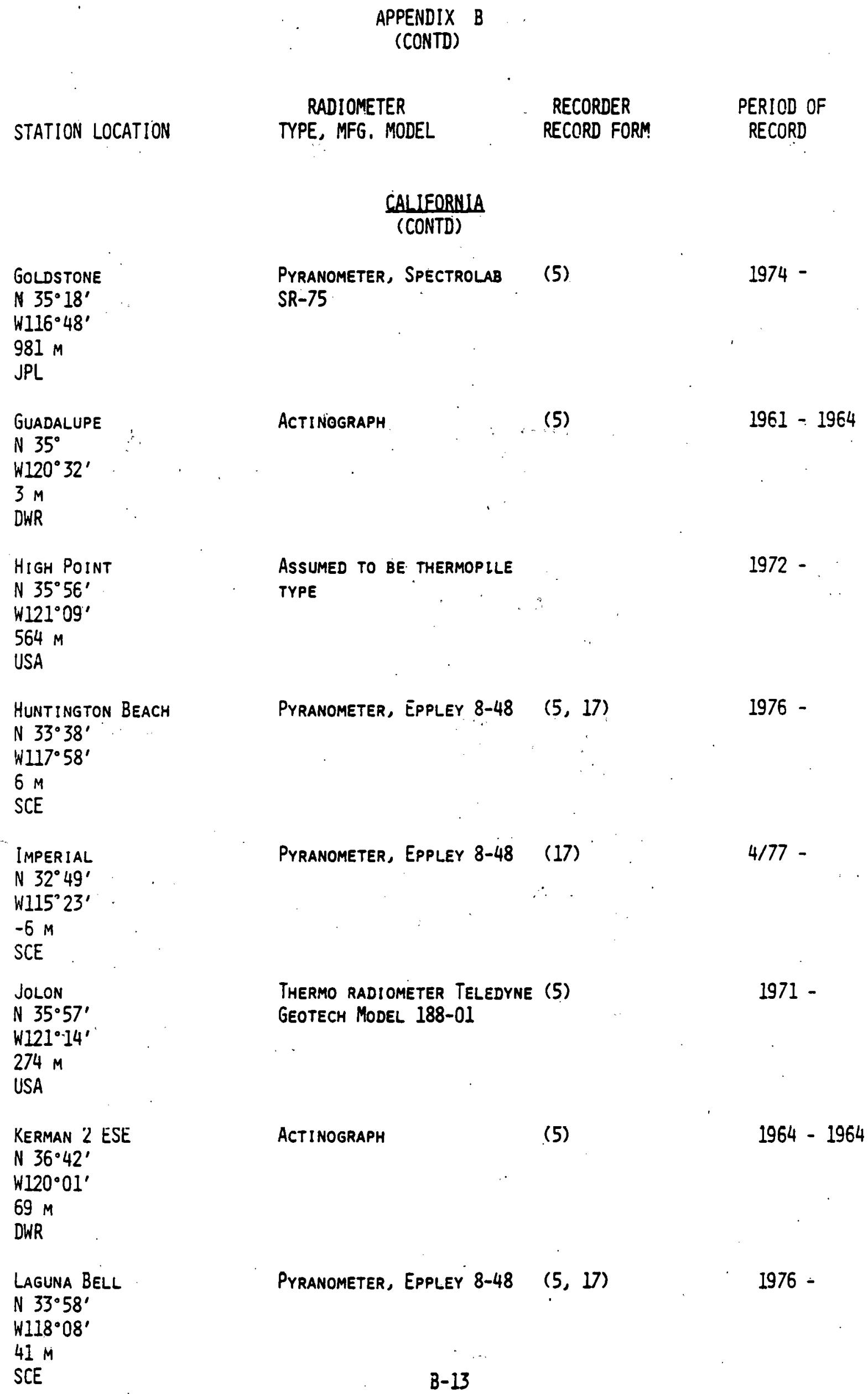




\section{RADIOMETER}

TYPE, MFG. MODEL
RECORDER

RECORD FORM
PERIOD OF

RECORD

\section{CALIFORNIA} (CONTD)

LA JOLLA

N $32^{\circ} 50^{\prime}$

W11 $7^{\circ} 15^{\prime}$

$26 \mathrm{M}$

DWR

Lake Mead

N $35^{\circ} 59^{\circ}$

$W 114^{\circ} 51^{\prime}$

$770 \mathrm{M}$

USGS

LANCASTER

N $34^{\circ} 42^{\prime}$

W11 $8^{\circ} 09^{\prime}$

714 M

SCE

LIVERMORE

N $37^{\circ} 41^{\prime}$

$W 121^{\circ} 47^{\circ}$

207 M

Sandia Labs

LIVERMORE

N $37^{\circ} 40^{\prime}$

W121 $50^{\circ}$

$152 \mathrm{M}$

LLL/BAAPC

LoMPOC

N $34^{\circ} 36^{\prime}$

$W 120^{\circ} 27^{\prime}$

$152 \mathrm{M}$

USDA

LONG BEACH

N $33^{\circ} 46^{\prime}$

W118 $11^{\prime}$

$11 M$

CA State University

AsSUMEd TO BE THERMOPILE

TYPE

$(5,24)$

$1928-1950$

Pyranometer, Eppley

(5)

$1952-1953$
Pyranometer, Eppley 8-48

PyRHELIOMETER, EPPLEY NIP
$(5,17)$

1976 -

Pyranometer, Spectrolab

$(5,17)$

1974 -
EPPLEY NIP

Pyranometer, Éppley 8-48

Pyranometer, Eppley LightBULB TYPE 50 THERMOPILE JUNCATION

ASSUMED TO BE THERMOPILE

TYPE

Pyranometer-Kahl SCI

instrument Corp. Star
(5)

(5)

1974 -

$1971-1973$

(5)

1971 - 


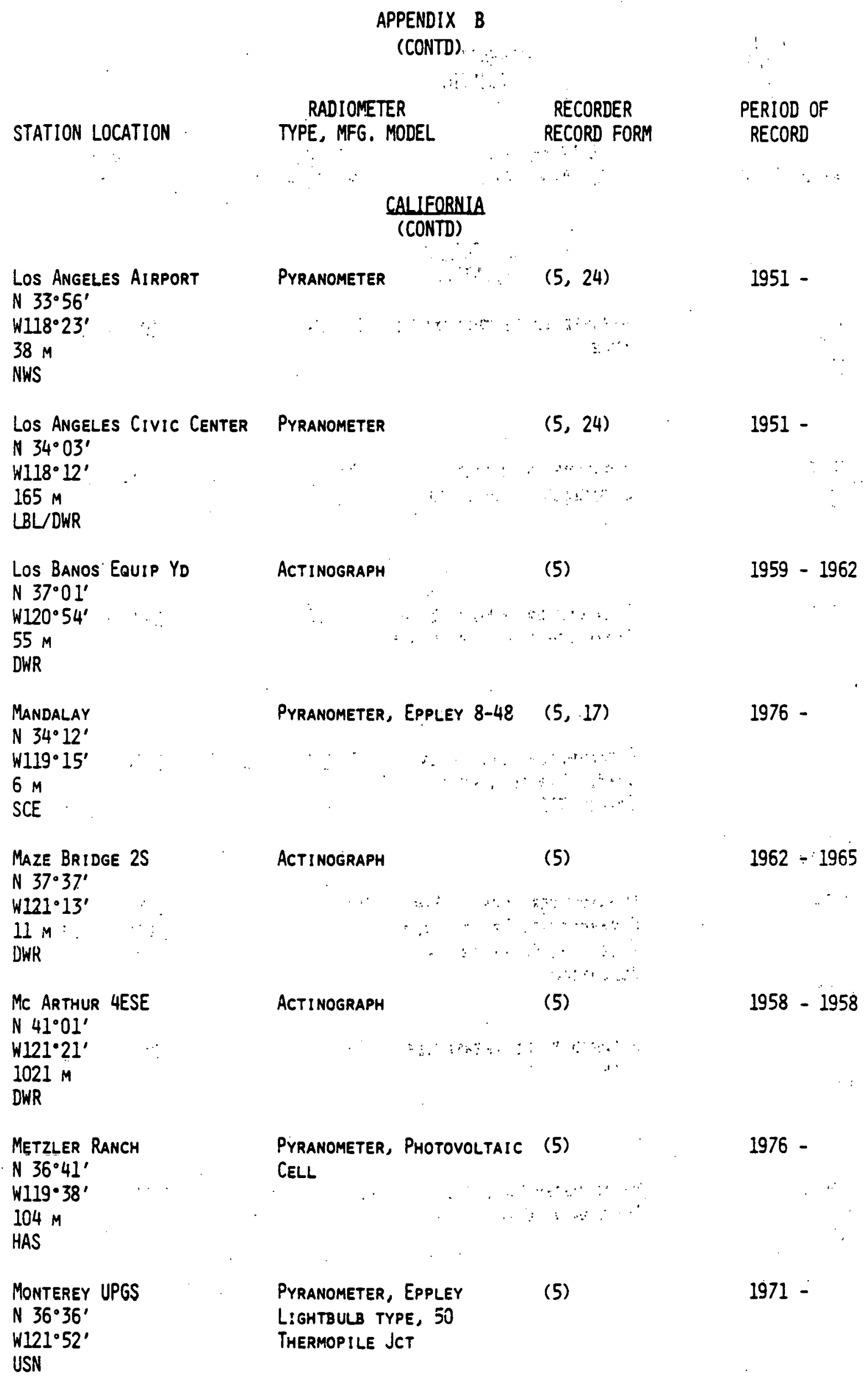




\begin{tabular}{|c|c|c|c|}
\hline \multirow{3}{*}{ STATION LOCATION } & $\begin{array}{l}\text { APPENDIX B } \\
\text { (CONTD) }\end{array}$ & & \\
\hline & $\begin{array}{c}\text { RADIOMETER } \\
\text { TYPE, MFG. MODEL }\end{array}$ & $\begin{array}{l}\text { RECORDER } \\
\text { RECORD FORM }\end{array}$ & $\begin{array}{l}\text { PERIOD OF } \\
\text { RECORD }\end{array}$ \\
\hline & $\frac{\text { CALLFORHIA }}{\text { (CONTD) }}$ & $\because$ & \\
\hline $\begin{array}{l}\text { MOORPARK } \\
\text { N } 34^{\circ} 17^{\prime} \\
\text { W11 } 8^{\circ} 54^{\prime} \\
141 \text { M } \\
\text { SCE }\end{array}$ & Pyranometer, EpPley 8-48 & $\begin{array}{c}(5,17) \\
.\end{array}$ & \\
\hline $\begin{array}{l}\text { NAPA } \\
\text { N } 38^{\circ}-177^{\prime} \\
\text { W122 } 17^{\prime} \\
5 \mathrm{M} \\
\text { BAAPC }\end{array}$ & $\begin{array}{l}\text { PYRANOMETER, EPPLEY } \\
\text { LIGHTBULB TYPE } 50 \\
\text { THERMOPILE JCT }\end{array}$ & $(5)$ & $1972-1974$ \\
\hline $\begin{array}{l}\text { NEWVILLE IE } \\
\text { N } 39^{\circ} 48^{\prime} \\
\text { W122 }{ }^{\circ} 1^{\prime} \\
198 \mathrm{M}^{\prime} \\
\text { DWR }\end{array}$ & ACTINOGRAPH $\quad \therefore$ & $\begin{array}{l}(5) \\
\ddots \\
\ddots\end{array}$ & $1966-1970$ \\
\hline $\begin{array}{l}\text { NORTHRI DGE } \\
\text { N } 34^{\circ} 14^{\prime} \\
\text { W11 }{ }^{\circ} 32^{\prime} \\
261 \text { M } \\
\text { CA STATE UNIVERSITY }\end{array}$ & $\begin{array}{l}\text { PYRANOMETER, MOLL- } \\
\text { GORCZYNSKI }\end{array}$ & (5) & $1963-$ \\
\hline $\begin{array}{l}\text { OAKLAND } \\
\text { N } 37^{\circ} 47^{\prime} \\
\text { W122 } \\
30 \mathrm{M} \\
\text { BAAPC }\end{array}$ & $\begin{array}{l}\text { Assumed TO BE THERMOPILE } \\
\text { TYPE }\end{array}$ & $(5)$ & $1970-1971$ \\
\hline $\begin{array}{l}\text { OLD RIVER } 3 S \\
N 35^{\circ} 13^{\prime} \\
\text { W11 } \\
1029^{\circ} 06^{\prime} \\
\text { DWR } \\
\text { DWR }\end{array}$ & ACTINOGRAPH & (5) & $1965-1967$ \\
\hline $\begin{array}{l}\text { PaCific Grove } \\
\text { N } 36^{\circ} 38^{\prime} \\
\text { W121 } \\
\text { Hopkins Marine Statio }\end{array}$ & ACTINOGRAPH & 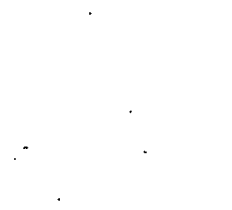 & \\
\hline
\end{tabular}




\section{APPENDIX B \\ (CONTD)}

\section{RADIOKETER}

TYPE, MFG. MODEL
RECORDER

RECORD FORM
PERIOD OF RECORD

\section{CALIFORNIA (CONTD)}

PALM SPRINGS

Pyranometer, Eppley $8-48 ;(5,17)$

N $33^{\circ} 47^{\prime}$

$W 116^{\circ} 28^{\prime}$

$93 \mathrm{M}$

SCE

PardeE

N $34^{\circ} 27^{\prime}$

W118 $35^{\prime}$

$315 \mathrm{M}$

SCE

PITTSBURG
N $38^{\circ} 02^{\prime}$
$W 121^{\circ} 51^{\prime}$
$30 \mathrm{M}$
BAAPC
PLACERVILLE IFG
$N 38^{\circ} 44^{\prime}$
W120 $44^{\prime}$
840 M
USBR
POINT MUGU
N $34^{\circ} 07^{\prime}$
W119 $06^{\prime}$
USN
RED BLUFF
N $40^{\circ} 09^{\prime}$
WI22 $15^{\circ}$
IO4 M
PG\&E/LBL

Pyranometer, Eppley light- (5)

BULB TYPE, 50 THERMOPILE JUNCTION

Pyranometer, Eppley 8-48 $(5,17) 1976$ -

Pyranometer, Photovoltalc :(5)

$1976-$

CELL

$1970-1974$

Pyranometer, Eppley $8-48 \cdot(5,28) \quad 1966$ -

Pyranometer, Eppley 8-48 Mag tape 7/71-

ACTINOGRAPH

(5)

$1967-1969$

N $40^{\circ} 10^{\circ}$

$W 122^{\circ} 08^{\prime}$

$85 \mathrm{M}$

DWR

(28) 


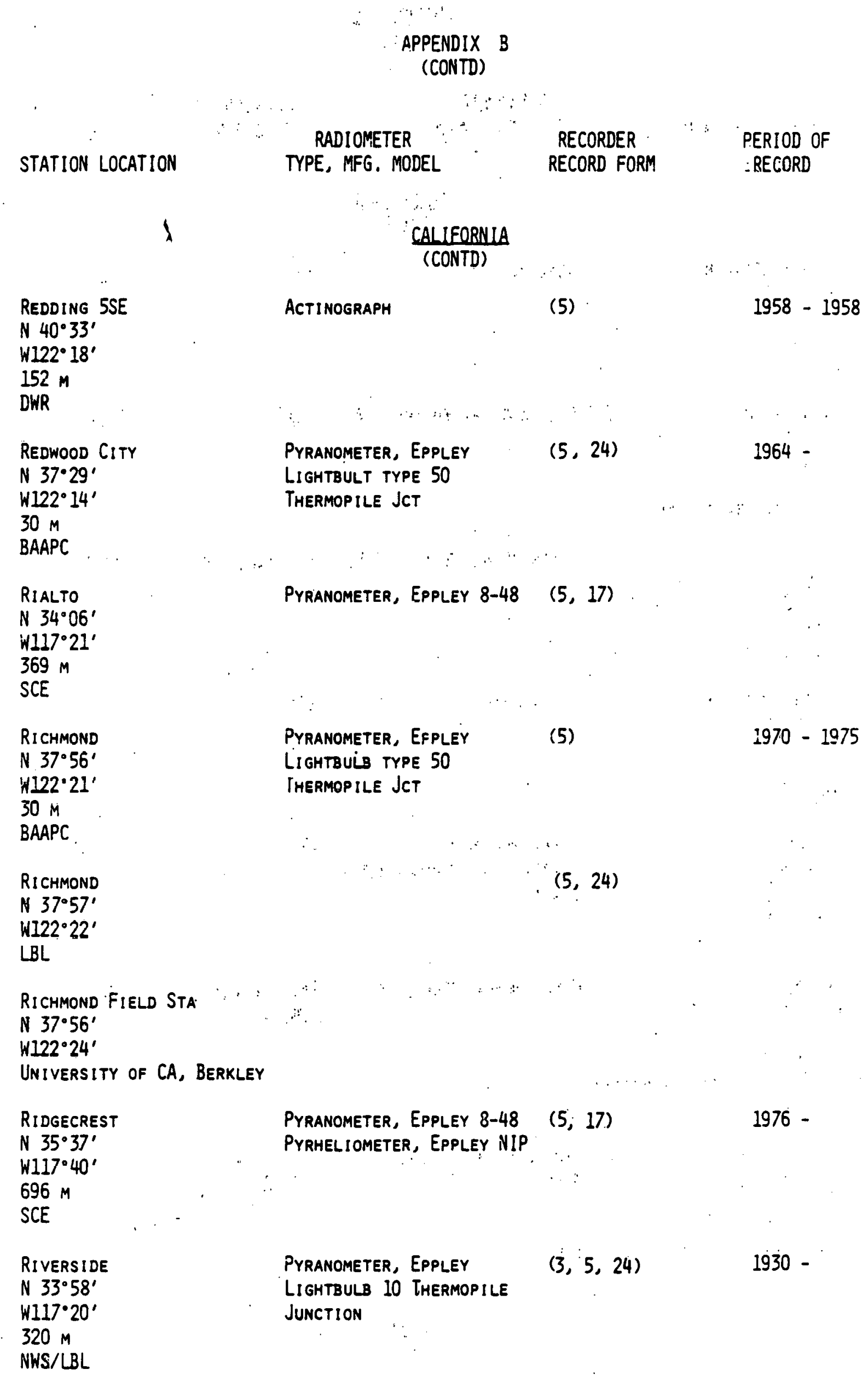


APPENDIX B

(CONTD)

RADIOMETER

STATION LOCATION

TYPE, MFG. MODEL

\section{CALIFORNLA \\ (CONTD)}

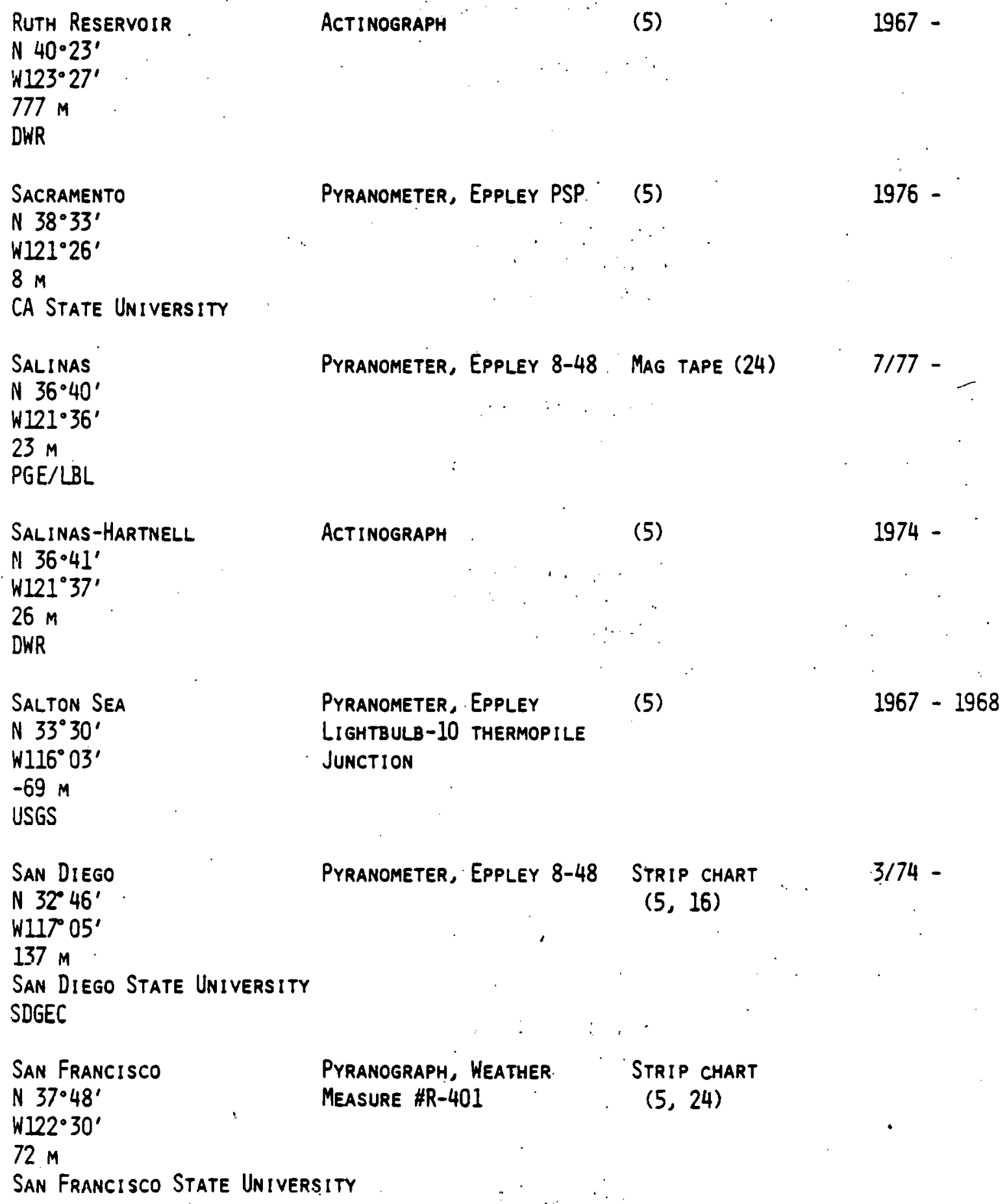

RECORDER

RECORD FORM
PERIOD OF

RECORD
1976 -

N $38^{\circ} 33$

$W 121^{\circ} 26^{\prime}$

$8 M$

CA State University

SALINAS

N $36^{\circ} 40^{\circ}$

W12 $1^{\circ} 36^{\prime}$

$23 \mathrm{M}$

PGE/LBL

Salinas-HartNell

il $36^{\circ} 41^{\prime}$

$W 121^{\circ} 37^{\prime}$

$26 \mathrm{~m}$

DWR

SALTON SEA

Pyranometer, Eppley

LIGHTBULB-10 THERMOPILE JUNCTION

Pyranometer, Eppley $8-48$

(5, 16) 


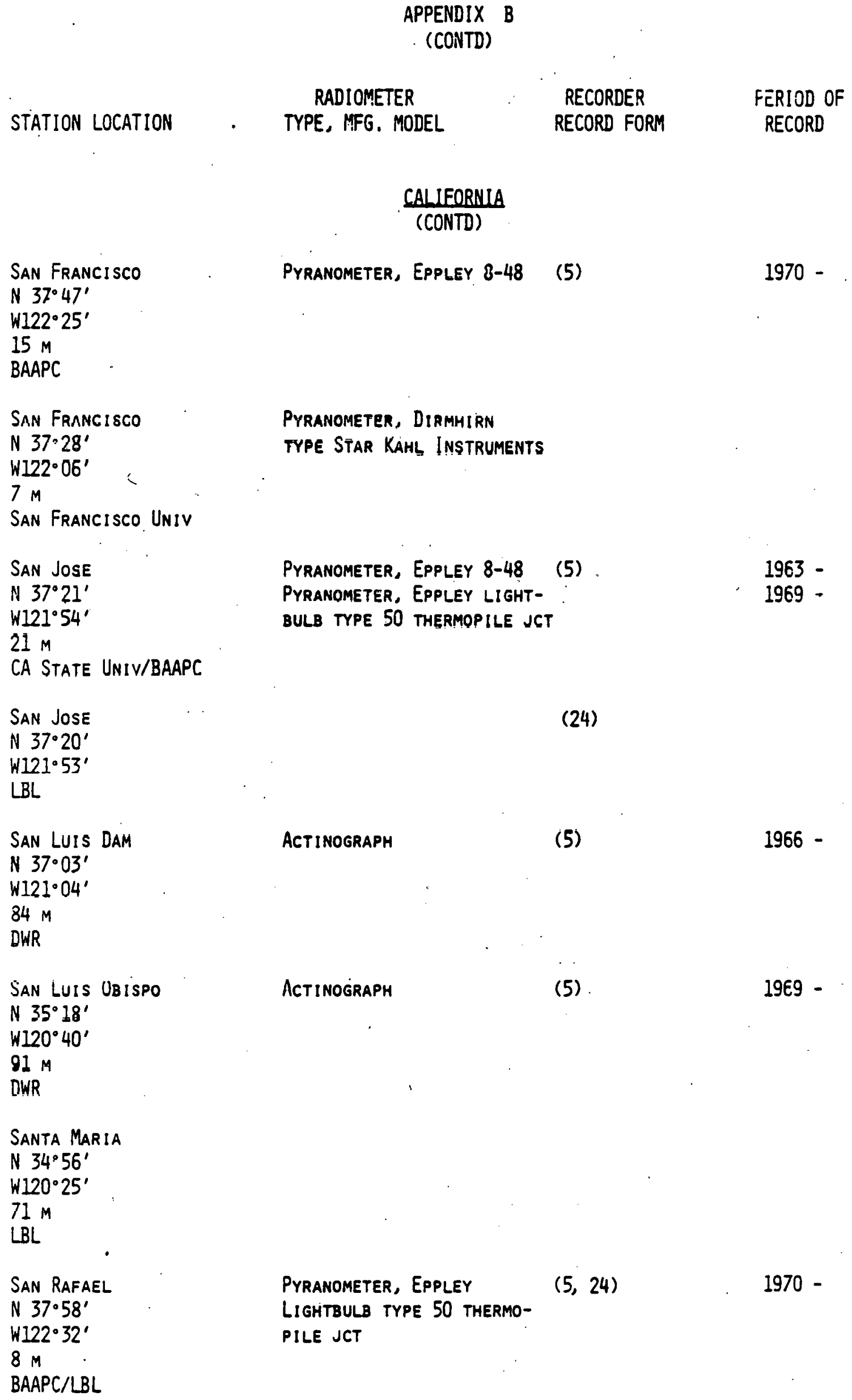

RADIOMETER

STATION LOCATION - TYPE, MFG. MODEL

CALIFORNIA

(CONTD)

San Francisco

N $37^{\circ} 47^{\prime}$

$W 122^{\circ} 25^{\prime}$

$15 \mathrm{M}$

BAAPC

SAN Francisco

N $37 \cdot 28^{\prime}$

W122 $06^{\prime}$

$7 \mathrm{M}$

San Francisco Univ

SAN Jose

N $37^{\circ} 21^{\prime}$

$W 121^{\circ} 54^{\prime}$

$21 M$

CA State Univ/BAAPC

SAN Jose

N $37^{\circ} 20^{\prime}$

W121 $53^{\prime}$

$\mathrm{LBL}$

SAN LUIS DaM

N $37^{\circ} 03^{\prime}$

W121.04'

$84 \mathrm{M}$

DWR

SAN LUIS UBISPO

N $35^{\circ} 18^{\prime}$

$W 120^{\circ} 40^{\prime}$

$91 \mathrm{M}$

DWR

Santa Maria

N $34^{\circ} 56^{\prime}$

$W 120^{\circ} 25^{\prime}$

$71 \mathrm{M}$

LBL

San Rafael

N $37^{\circ} 58^{\prime}$

$W 122^{\circ} 32^{\circ}$

$8 M$

BAAPC/LBL

RECORDER

RECORD FORM

FËRIOD OF

RECORD

Pyranometer, Dirmhizn

type Star Kahl Instruments

Pyranometer, Eppley 8-48 (5)

Pyranometer, Eppley lightBULB TYPE 50 THERMOPILE JCT

1963 -

1969 -

ACTINOGRAPH

(5)

1966 -

ACtinOGRAPH

(5)

$19 E 9$ -

Pyranometer, Eppley

LIGHTBULB TYPE 50 THERMO-

PILE JCT

$(5,24)$

$1970-$ 
APPENDIX B

(CONTD)

STATION LOCATION
RADIOMETER

TYPE, MFG. MODEL $\because$ RECORD FORM
PERIOD OF

RECORD

\section{CALIEORNIA}

(CONTD)

SAN VICENTE

N $32^{\circ} 55^{\prime}$

$W 116^{\circ} 55^{\prime}$

$201 M$

USGS

SAN VICENTE RES

N $32^{\circ} 55^{\prime}$

$W 116^{\circ} 55^{\prime}$

$201 \mathrm{M}$

LBL

SANDY BEACH

N $33^{\circ} 11^{\prime}$

$W 115^{\circ} 50^{\prime}$

$-69 M$

USGS

SANTA Rosa

N $38^{\circ} 31^{\prime}$

W122 $43^{\prime}$

$137 \mathrm{M}$

BAAPC

SANTA Rosa

N $38^{\circ} 27^{\prime}$

$W 122^{\circ} 42^{\prime}$

$51 \mathrm{M}$

SCHRAMM RANCH

N $36^{\circ} 34^{\prime}$

W120 $08^{\prime}$

$54 \mathrm{M}$

HAS

SHAFTER
$N 35^{\circ} 32^{\prime}$
$W 119^{\circ} 17^{\prime}$
$94 \mathrm{M}$
USDA
SODA SPRINGS
N $^{\circ} 39^{\circ} 20^{\prime}$
W120 $22^{\prime}$
$2099^{\prime} M$
USFS

Pyranometer, Eppley

(5)

$1957-1959$
LABORATORY LIGHTBULB TYPE

10 THERMOPILE JCT
ASSUMED TO BE THERMOPILE: TYPE

ASSUMED TO BE THERMOPILE

(5)

TYPE

Pyranometer, Eppley 8-48 Mag tape

Pyranometer, Solameter
Photovoltaic Cell

ASSUMED TO BE THERMOPILE (5)

TYPE

(5)

Pyranometer, Eppley light-: (5)

BULB TYPE 50 THERMOPILE JCT
$10 / 77$. -

$1975-1975$

$1975-$

$1961-1962$

1972

1946 - 


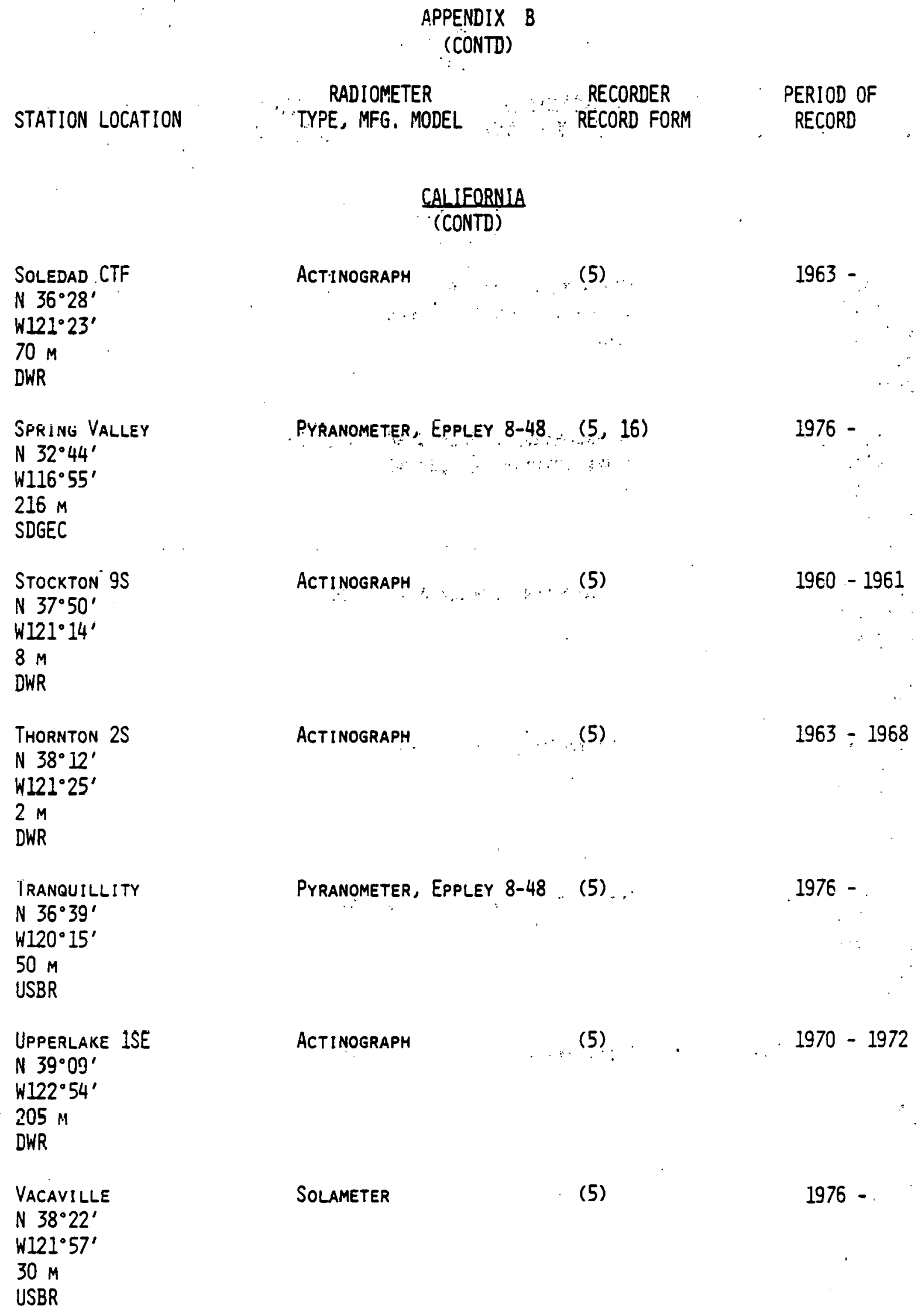




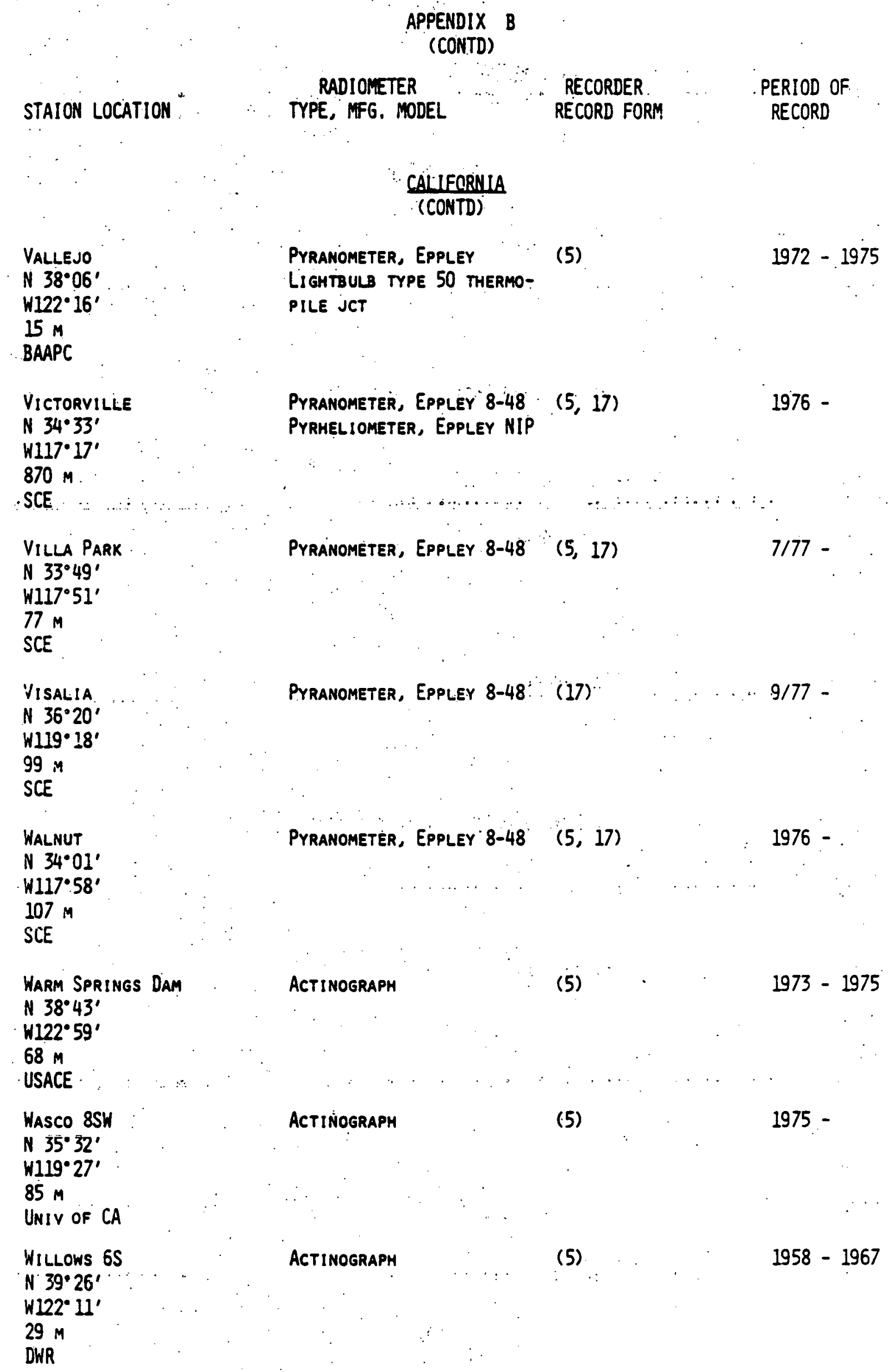




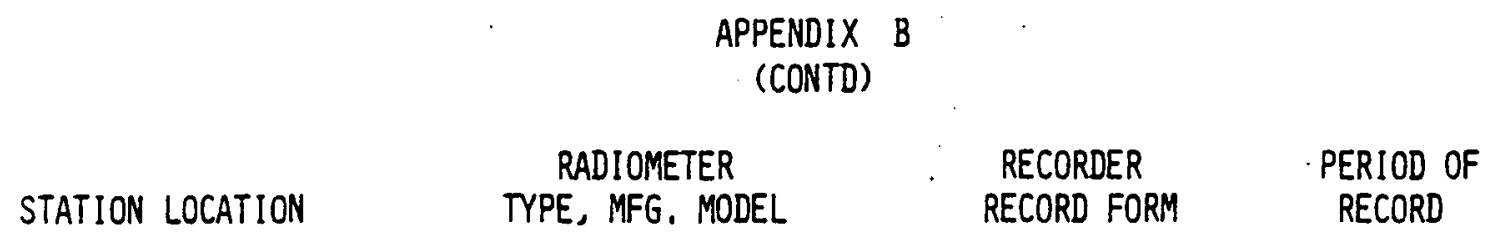

$\frac{\text { CALIFORNLA }}{\text { (CONTD) }}$

YucCa Valley

Pyranometer, Eppley 8-48

$(5,17)$

1976

N $34.07^{\prime}$

W116 $25^{\prime}$

$1024 \mathrm{M}$

SCE

Pyrmeliometer, Eppley NIP

\section{COLORADO.}

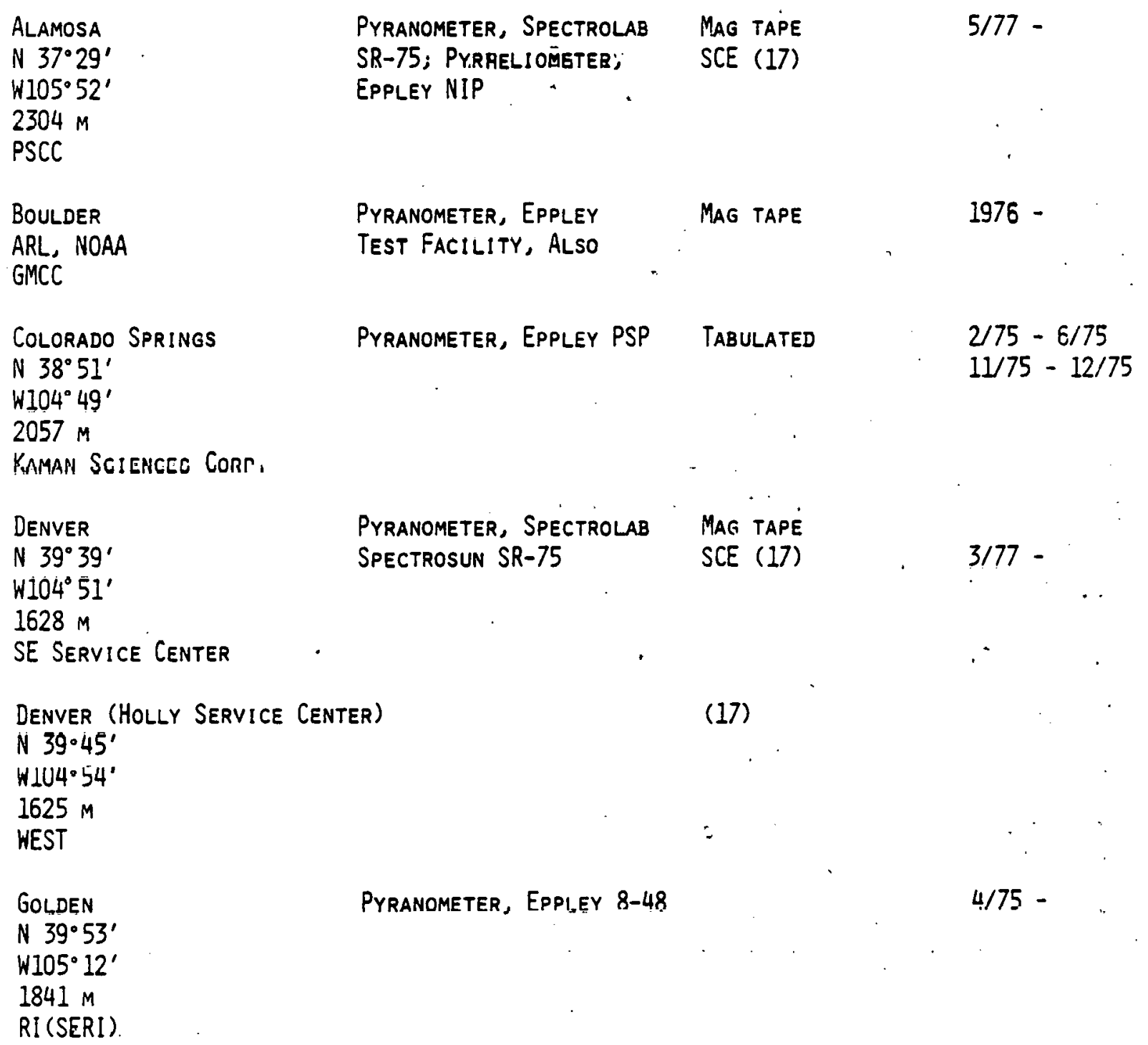




\section{STATION LOCATION}

RADIOMETER

TYPE, MFG. MODEL

\section{COLORADO}

(CONTD)

Pueblo.

N $38^{\circ} 16^{\prime}$

W10 '37'

$1481 \mathrm{M}$

Public Service co of CO

USAF ACA DEMY

N $39^{\circ}$

N105

$1981 \mathrm{M}$

USAF

Avery PoInt (Groton)

COAST GUARD

AvoN

iv $41^{\circ} 49^{\prime} \quad \therefore$

$W 72^{\circ} 48^{\prime}, \quad:$.

$285 \mathrm{M}$

TMSC

BLOOMF IELD

BRIDGEPORT

N $73^{\circ} O E^{\prime}$

W $41^{\circ} 12^{\prime}$

$11 \mathrm{M}$

NU

CoVEntRY

UNIV OF CT

DANBURY
Pyranometer, Spectrolab

SPECTROSUN, SR-75

\section{CONNECIICUI}

Pyranometer, Matrix, Inc.

SOL-A-METER

Pyranometer, Eppley 8-48

Pyranograph, Belfort

Pyrhel IOMETER, EPPLEY NIP

Pyranometer

Pyranometer, EPPLEY

Pyranometer, Matrix
APPENDIX B

(CONTD)

RECORDER

RECORD FORM

PERIOD OF

RECORD
Pyranometer, Eppley PSP

TELETYPE

ASR 33
$3 / 77-$

SCE (17)

11/75 -

$8 / 74-$

STRIP CHART

$7 / 73-$

CLIMATRONICS FUED

บ:58 -

15 MIN MAGNETIC

DISK

$7 / 78$ -

$6 / 75-$

Pyranometer, Eppley 8-48 Strip chart

$7178-$

Pyrhel tometer, Eppley NIP. Climatronics fleo

15 MIN Magnetic tape

$1976-$ 


\begin{tabular}{|c|c|c|c|}
\hline STATIUN LOCATION & $\begin{array}{c}\text { RADIOMETER } \\
\text { TYPE, MFG. MODEL }\end{array}$ & $\begin{array}{l}\text { RECORDER } \\
\text { RECORD FORY }\end{array}$ & $\begin{array}{l}\text { PERIOD OF } \\
\text { RECORD }\end{array}$ \\
\hline & $\frac{\text { CONNECTICITI }}{\text { (CONTD) }}$ & & - \\
\hline DERBY & Pyranometer, Matrix, Inc. & & $1976-$ \\
\hline ENFIELD & $\begin{array}{l}\text { Pyranometer, Matrix, } \\
\text { InC. SOL-A-METER }\end{array}$ & & $8 / 75-$ \\
\hline HARTFORD & $\begin{array}{l}\text { Pyranometer, Matrix, } \\
\text { Inc. Sol-a-meter }\end{array}$ & & $9 / 75$ - \\
\hline $\begin{array}{l}\text { Mi DDLETOWN (MAROMAS) } \\
\text { N } 72^{\circ} 34^{\prime} \\
\text { W } 41^{\circ} 32^{\prime} \\
18 \mathrm{M} . \\
\text { iNU }\end{array}$ & $\begin{array}{l}\text { Pyranometer, EPPLEY 8-48 } \\
\text { PYRHELIOMETER, EPPLEY NIP }\end{array}$ & $\begin{array}{l}\text { StRiP ChART } \\
\text { CLIMATRONICS F } 460 \\
15 \text { MIN MAGNeTIC TAPE }\end{array}$ & $\begin{array}{l}8 / 73- \\
7 / 78- \\
\end{array}$ \\
\hline MIDDLETOWN & $\begin{array}{l}\text { PYAANMOMETER, MATRIX, } \\
\text { INC. SOL-A-METER }\end{array}$ & & 1976 - \\
\hline $\begin{array}{l}\text { MOUNt CARMEL } \\
\text { State EXPERIMENTAL FARM }\end{array}$ & Pyranograph & & 1959 - \\
\hline NEW BRITAIN & $\begin{array}{l}\text { Pyranometer, MATRIX, } \\
\text { INC. SOL-A-METER }\end{array}$ & & 1976 - \\
\hline New Haven & $\begin{array}{l}\text { PYranometer, MATRIX, } \\
\text { INC, SOL-A-METER }\end{array}$ & & $10 / 75-$ \\
\hline STAMFORD & $\begin{array}{l}\text { Pyramometer, MATRIX, } \\
\text { InC. SOL-A-METER }\end{array}$ & . & $8 / 75-$ \\
\hline WATERBURY & $\begin{array}{l}\text { Pyranometer, Matrix, } \\
\text { Imc. SOl-A-Meter }\end{array}$ & & $8 / 75-$ \\
\hline $\begin{array}{l}\text { WATERFord (MILLSTONE } \\
\text { N } 72^{\circ} 10^{\prime} \\
\text { N } 41^{\circ} 18^{\prime} \\
6 \mathrm{M}, 23 \mathrm{M} \\
\text { NU }\end{array}$ & $\begin{array}{l}\text { PYRANOMETER; EPPLEY } 8=48 \\
\text { PYRHELIOMETER EPPLEY NIP }\end{array}$ & $\begin{array}{l}\text { Climatronies F } 460 \\
15 \text { MIN MAGNetIC TAPE }\end{array}$ & $\begin{array}{l}7 / 73- \\
6 / 78-\end{array}$ \\
\hline
\end{tabular}




\begin{tabular}{|c|c|c|c|}
\hline \multirow[b]{2}{*}{ STATION LOCATION } & $\begin{array}{l}\text { APPENDIX } \\
\text { (CONTD) }\end{array}$ & & \multirow[b]{2}{*}{$\begin{array}{l}\text { PERIOD OF } \\
\text { RECORD }\end{array}$} \\
\hline & $\begin{array}{l}\text { RADIOMETER } \\
\text { TYPE, MFG. MODEL }\end{array}$ & $\begin{array}{l}\text { RECORDER } \\
\text { RECORD FORM }\end{array}$ & \\
\hline & $\therefore$ & & \\
\hline & DELLAWARE & & \\
\hline $\begin{array}{l}\text { NEWARK } \\
\text { UNIV OF DE }\end{array}$ & $\begin{array}{l}\text { Pyranometers }(2),(T) \text {, } \\
\text { EPPLEY } 8-48\end{array}$ & TABULATED HOURLY & $\begin{array}{r}1 / 74- \\
11 / 74-\end{array}$ \\
\hline
\end{tabular}

DISTRICT OF COLUMBIA.

WASHINGTON

i) $38^{\circ}$

W $77^{\circ}$

SRL

Pyranometer, Eppley PSP: . Spectral published 9/68 - 11/72

(1)

ELORIDA

Cape Canaveral

N $28^{\circ} 25^{\prime}$

in $80^{\circ} 36^{\prime}$

$9 \mathrm{M}$

fl Solar Energy. Center

Gainesville

N $29^{\circ} 41^{\prime}$

W $82^{\circ} 16^{\prime}$

$47 \mathrm{M}$

UNIVER OF FL

Pyranometer, Eppley 8-48 Strip chart 1954 -

Pyranometer, Eppley 10- (18)

JUNCTION

1965 -

PyRHELIOMETER, EPPLEY NIP 


\begin{tabular}{|c|c|c|c|}
\hline \multirow[b]{2}{*}{ STATION LOCATION } & \multicolumn{2}{|l|}{$\begin{array}{l}\text { APPENDIX B } \\
\text { (CONTD) }\end{array}$} & \multirow[b]{2}{*}{$\begin{array}{l}\text { PERIOD OF } \\
\text { RECORD }\end{array}$} \\
\hline & $\begin{array}{l}\text { RADIOMETER } \\
\text { TYPE, MFG. MODEL }\end{array}$ & $\begin{array}{l}\text { RECORDER } \\
\text { RECORD FORM }\end{array}$ & \\
\hline \multicolumn{4}{|c|}{$\frac{\text { ELORIDA }}{\text { (CONTD) }}$} \\
\hline $\begin{array}{l}\text { J.F.K. SPACE CENTER } \\
\text { COMPLEX } 39_{B}\end{array}$ & $\begin{array}{l}\text { MK III YELLOTT, EQUatorial } \\
\text { MOUNT } \\
\text { MK-I-G YELIOTT }\end{array}$ & STRIP CHART & $6 / 66-1977$ \\
\hline COMPLEX $39_{A}$ & $\begin{array}{l}\text { MK-I-G YELLOTT } \\
\text { MK III YELLOTT, EQUATORIAL } \\
\text { MOUNT } \\
\text { MK-I-G YELLOTT }\end{array}$ & & $\begin{array}{l}6 / 66-1977 \\
6 / 66-1977 \\
1966-1077\end{array}$ \\
\hline RURAL Station. & Pyranometer & & $1965-1977$ \\
\hline \multicolumn{4}{|c|}{ GEORGIA } \\
\hline $\begin{array}{l}\text { ATLANTA } \\
N 33^{\circ} 46^{\prime} \\
\text { W } 84^{\circ} 25^{\prime} \\
335 \text { M } \\
\text { GEORGIA TECH UNIV } \\
\text { (DOE - TRAINING SITE) }\end{array}$ & $\begin{array}{l}\text { PYRANOMETERS (5), EPPLEY } \\
\text { PSP; PYRHELIOMETERS ( } 2 \text { ), } \\
\text { EPPLEY NIP } \\
\text { CSIRO-PYRRADIOMETER }\end{array}$ & $\begin{array}{l}\text { ACUREX } \\
\text { Autodata } 9\end{array}$ & $\begin{array}{c}\text { FALL } 78- \\
.\end{array}$ \\
\hline $\begin{array}{l}\text { ATLANTA } \\
N 33^{\circ} 22^{\prime} \\
W 84^{\circ} 47^{\prime} \\
280 \mathrm{~m} \\
\text { SANDIA LABS }\end{array}$ & $\begin{array}{l}\text { PYRANOMETER, EPPLEY PSP } \\
\text { PYRHELIOMETERS (2), } \\
\text { EPPLEY NIP. }\end{array}$ & $\begin{array}{l}\text { Digital EG\&G } \\
\text { Custom }\end{array}$ & $9 / 1 / 77-$ \\
\hline \multicolumn{4}{|c|}{ HAWALI } \\
\hline 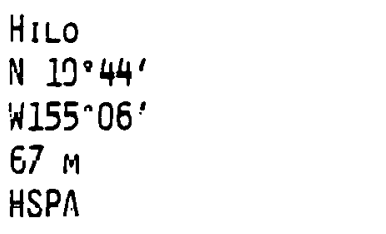 & Pyranometer, Eppley 8-48 & L\&N SPEEDOMAX $W$ & $1960-$ \\
\hline $\begin{array}{l}\text { HOLMES } \\
\text { N } 21^{\circ} 18^{\prime} \\
W 157^{\circ} 49^{\prime} \\
23 \text { M } \\
\text { HNE! }\end{array}$ & Pyranometer, EpPley $8-48$ & $(26)$ & $3 / 76-$ \\
\hline
\end{tabular}




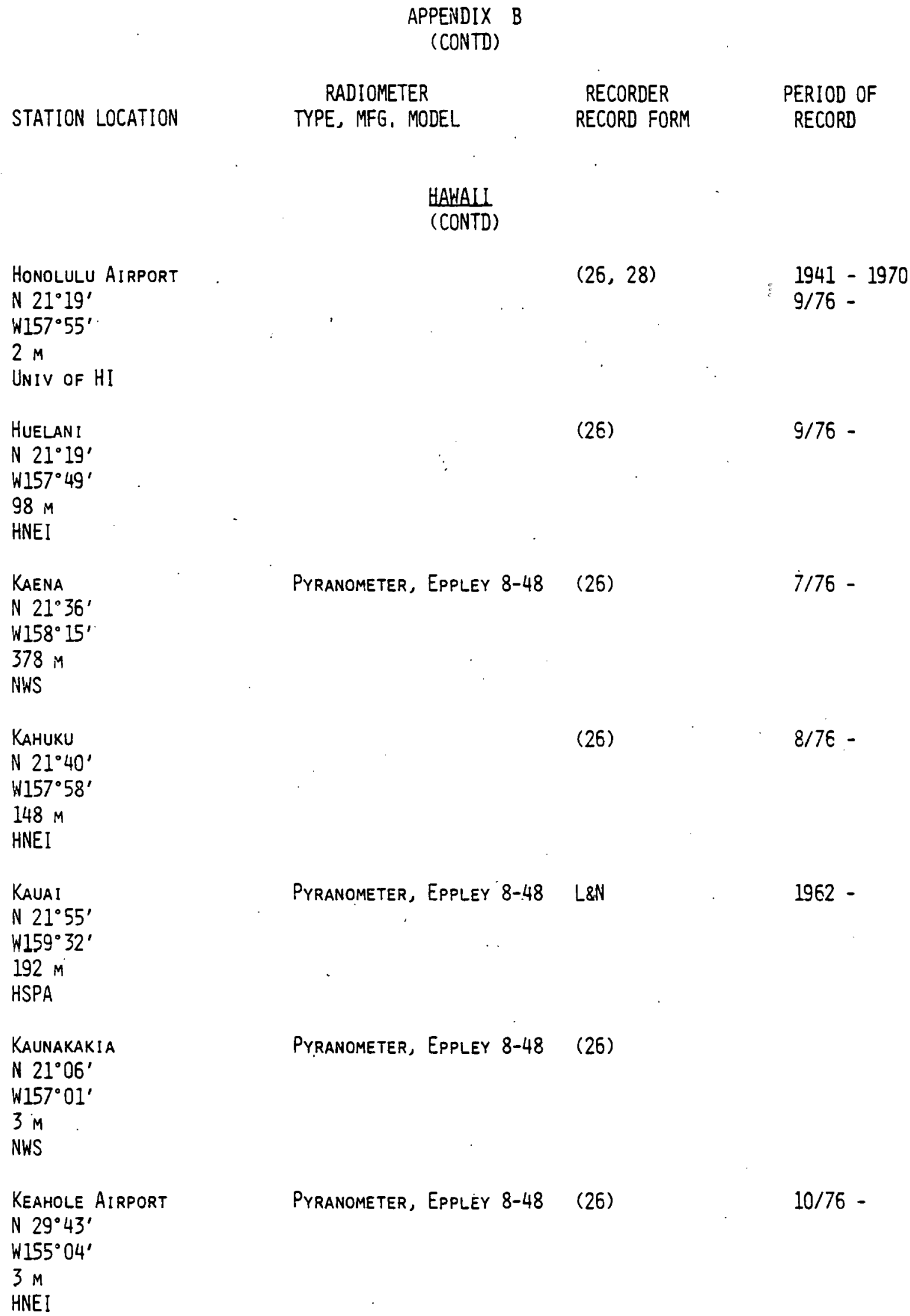

$(26,28)$

$1941-1970$

9/76 -

(2E)

$9 / 76-$

(26)

$7 / 76-$

(26)

8/76 -

Pyranometer, Eppley $8-48$ L8N

1962 -

Pyranometer, Eppley $8-48$ (26)

Pyranometer, Epplèy 8-48 (26) 10/76 - 


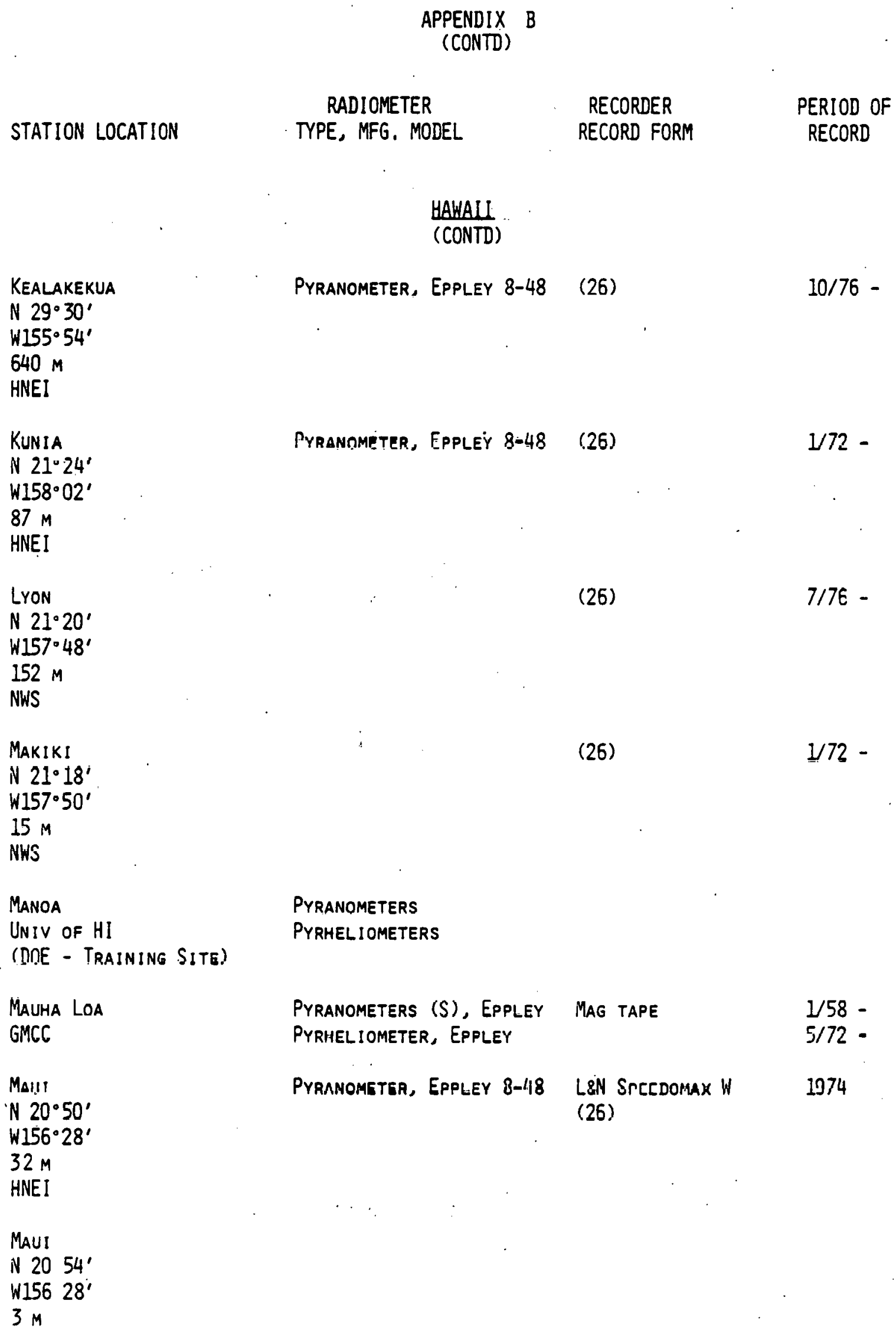

RADIOMETER

TYPE, MFG. MODEL

HAWALI

(CONTD)

KEALAKEKUA

N $29^{\circ} 30^{\prime}$

$W 155^{\circ} 54^{\prime}$

$640 \mathrm{M}$

HNEI

Kunia

N $21^{\circ} 24^{\circ}$

W158 $02^{\prime}$

$87 \mathrm{M}$

HNEI

LYON

N $21^{\circ} 20^{\prime}$

$W 157 \cdot 48^{\prime}$

152 M

NWS

MAKIKI

iv $21^{\circ} 18^{\prime}$

$W 157^{\circ} 50^{\prime}$

$15 \mathrm{M}$

NWS

Manoa

UNIV OF HI

(Dine - Traiming Site)

Mauha loa

GMCC

Mai!!

'N $20^{\circ} 50^{\prime}$

$W 156^{\circ} 28^{\prime}$

$32 \mathrm{M}$

HNEI

Maui

N $2054^{\prime}$

W156 28'

$3 \mathrm{M}$

RECORDER

RECORD FORM

PERIOD OF

RECORD

Pyranometer, Eppley 8-48 (26)

$10 / 76-$

Pyrannmeter, Eppley 8-48 (26)

$1 / 72-$

(26)

$7 / 76-$

(26)

$172-$

Pyranometers

Pyrhel IOMETERS

Pyranometers (S), Eppley Mag tape 1/58 -

PYRHELIOMETER, EPPLEY $5 / 72$ -

Pyramometea, Eppley 8-18 L8N Srecdomax W 1974

(26) 
APPENDIX B

(CONTD)

STATION LOCATION

RADIOMETER

TYPE, MFG, MODEL

HAWALL

(CONTD)

Maunawili

N $21^{\circ} 21^{\prime}$

$W 157^{\circ} 46^{\prime}$

$125 \mathrm{M}$

NWS

Mililani

N) $21^{\circ} 26^{\prime}$

$W 158^{\circ} 01^{\prime}$

$146 \mathrm{M}$

HNEI

MoLokal

N $21^{\circ} 18^{\prime}$

W $157^{\circ} 50^{\prime}$

$15 \mathrm{M}$

HSPA

DaHU

N $21^{\circ} 23^{\prime}$

$W 158^{\circ} 02^{\prime}$

$87 \mathrm{M}$

HSPA

Palehua

N $21^{\circ} 23^{\prime}$

H158.07'

$649 \mathrm{M}$

HNEI

Tantulus

N $21^{\circ} 20^{\prime}$

$W 157^{\circ} 49^{\prime}$

$610 \mathrm{M}$

NWS

WaIkIKI

N $21^{\circ} 17^{\prime}$

$W 157^{\circ} 50^{\prime}$

$3 \mathrm{M}$

HNEI
RECORDER

RECORD FORM

PERIOD OF

RECORD

(26)

(26)

$10 / 76$ -

Pyranometer; Eppley 8-48 L8N Speedomax W $1947-1975$

Pyranometer, Eppley 8-48 Lan Speedomax W 1963 -

(26)

$9 / 76-$

(26)

(26)

$9 / 76-$ 


\begin{tabular}{|c|c|c|c|}
\hline STATION LOCATION & $\begin{array}{c}\text { RADIOMETER } \\
\text { TYPE, MFG. MODEL }\end{array}$ & $\begin{array}{c}\text { RECORDER } \\
\text { RECORD FORM }\end{array}$ & $\begin{array}{l}\text { PERIOD OF } \\
\text { RECORD }\end{array}$ \\
\hline & $\frac{\mathrm{HAW}}{\mathrm{CCON}}$ & & \\
\hline $\begin{array}{l}\text { Waimano Home } \\
\text { N } 21^{\circ} 26^{\prime} \\
\text { W157 } \\
327^{\circ} 56^{\prime} \\
\text { HNEI } \\
\text { HEI }\end{array}$ & . & (26) & $9 / 76-$. \\
\hline
\end{tabular}

\section{ILLINOIS}

LEMONT

N $41^{\circ} 42^{\prime}$

W $87^{\circ} 59^{\prime}$

$227 \mathrm{M}$

argonne National Lab

Macomb

N $40^{\circ} 28^{\prime}$

W $90^{\circ} 40^{\prime}$

$231 \mathrm{M}$

WESTERN IL UNIV

URBANA

N $40^{\circ} 06^{\prime}$

W $88^{\circ} 14^{\prime}$

$226 \mathrm{M}$

IL State Water Survey
Pyranometers, Eppley Strip chart $1950-1975$

Pyranometer (D), EPpley TAPE Printout 1975 -

8-48

Pyranograph

Pyanamograph, heather

MeAsure R401
$1 / 73-$

STRIP CHART

$1966-$

\section{INDIANA}

Dusois

Ni $38^{\circ} 21^{\prime \prime}$

W $86^{\circ} 42^{\prime}$

$210 \mathrm{M}$

Purdue Univ

FARMLAND

Pyrheliometer, Eppley 50 Micro-Computer

8/76-

N $40^{\circ} 15^{\prime}$

Pyranometer, EPpley 50

Mir.Rn-Romputer

$3 / 76$

W $85^{\circ} 09^{\prime}$

$294 \mathrm{M}$

Purdue Univ 
RADIOMETER

TYPE, MFG, MODEL

\section{INALANA \\ (CONTD)}

WanataH

N $41^{\circ} 26^{\prime}$

W $86^{\circ} 56^{\circ}$

$224 \mathrm{M}$

Purdue Univ

West LafayetTe

N $40^{\circ} 28^{\circ}$

W $87^{\circ}$

$215 \mathrm{M}$

Purdue Univ
APPENDIX B

(CONTD)

Pyrheliometer, Eppley 50 Micro-Computer 3/76 -

Pyranometer, Eppley PSP

\section{KENTUCKY}

DRAKESBORO

N $37^{\circ} 16^{\prime}$

W $87^{\circ} 00^{\prime}$

$157 \mathrm{M}$

TVA

Paducah

iN $37^{\circ} 09^{\prime}$

W $88^{\circ} 46^{\prime}$

$111 \mathrm{M}$

TVA

Pyranometer, Eppley

8-48; Pyrradiometer,

TELEDYNE GEOTECH

TCH - 188-01

Pyranometer, Eppley 8-48, Mag tape nova 1200 Late 1975 Pyrradiometer, Teledyne Hewlett Packard 345 GEOTECH TCH - 188-01

\section{MAINE}

BOOTHBAY HARBOR

N $43^{\circ} 50^{\circ}$

W $69^{\circ} 38^{\prime}$

$0 \mathrm{M}$

BIGELOW LABORATORY

FOR OCEAN SCIENCES
RECORDER

RECORD FORM

PERIOD OF

RECORD
$3 / 68-$

Mag tape Nova $1200 \quad 1967-1978$

HewLETT PACKaRd 345
Pyranometer, Silicon

MATRIX MK 1-G
STRIP CHART

Climatronics (19)
PATCHY FROM

ע77 - 12/77

i/78 - 


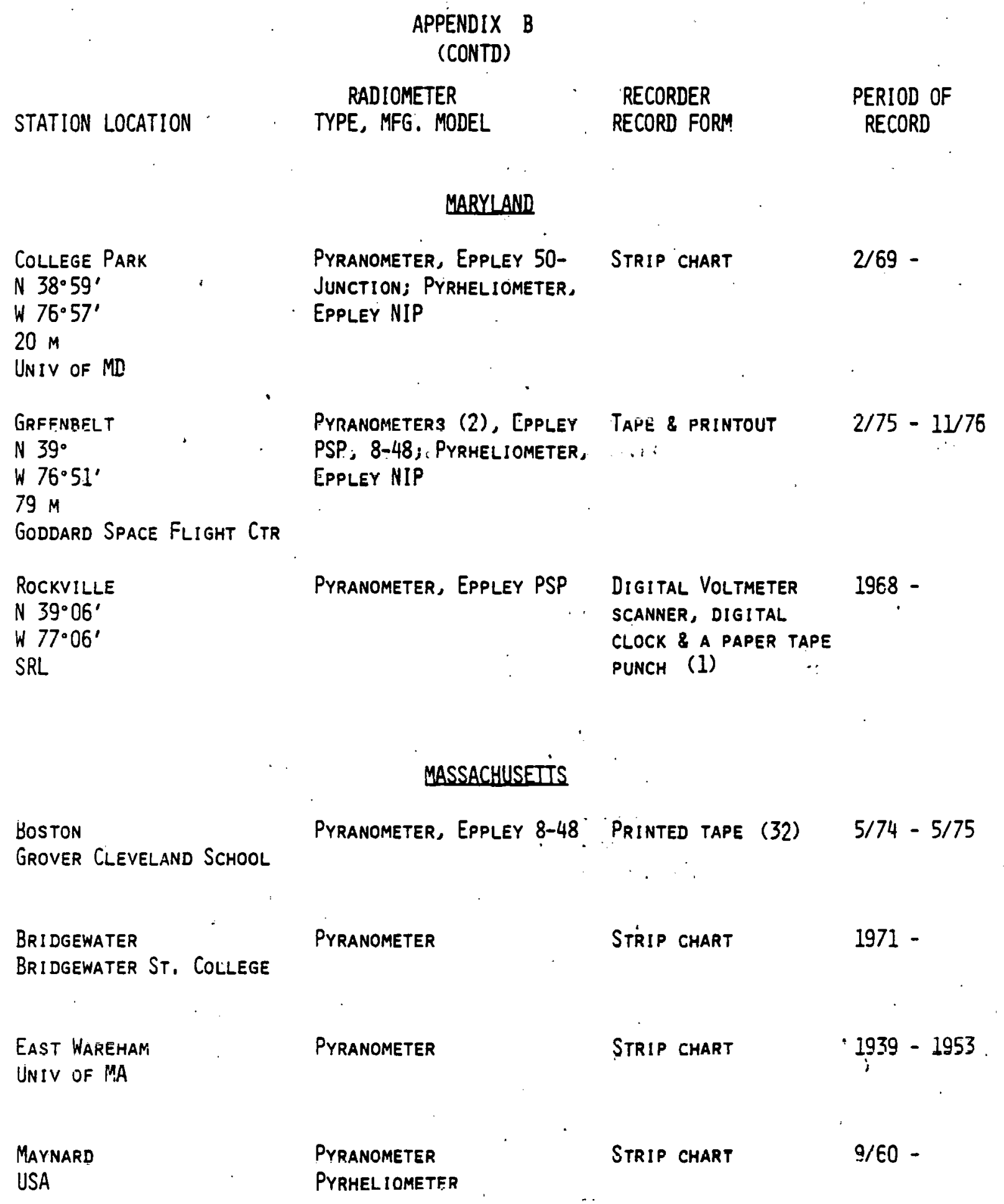




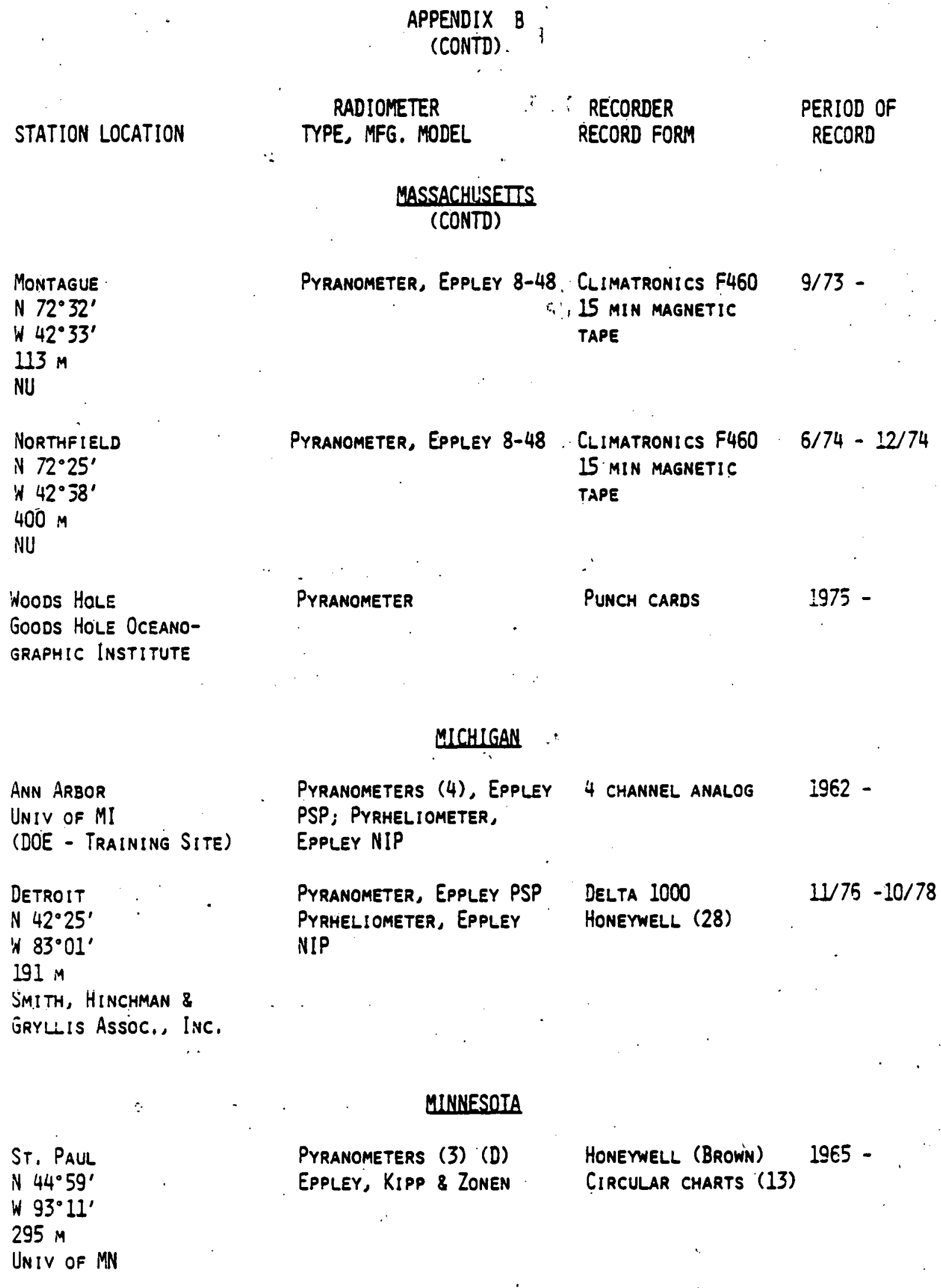
Pyranometer, Eppley $8-48$. Climatronics F460 6/74 - 12/74 15 MIN MAGNETIC TAPE

MICHIGAN :

ANN ARBOR

UNIV OF MI

Pyranometers (4), Eppley

4 CHANMEL ANALOG

$9 / 73-$

(DOE - Training Site)

PSP; PYRHELIOMETER,

EPPLEY NIP

DETROIT

Pyranometer, Eppley PSP Delta 1000

PyRHELlOMETER, EPPLEY HONEYWELl (28)

$11 / 75-10 / 78$

N $42^{\circ} 25^{\prime}$

N $83^{\circ} 01^{\prime}$

NIP

$191 \mathrm{M}$

SMITH, HINCHMAN \& GRYLLIS AsSOC., INC.

\section{MINNESOTA}

ST. PAUL

N $44^{\circ} 59^{\prime}$

W $93^{\circ} 11^{\prime}$

$295 \mathrm{M}$

UNIV OF MN

Pyranometers (3) (D)

EPPLEY, KIPP \& ZONEN

HONEYWELL (BrowiN)

CIRCULAR CHARTS (13) 
APPENDIX B

(CONTD)
RADIONETER

TYPE, MFG, MODEL
RECORDER

RECORD FORM
PERIOD OF

RECORD

\section{MISSISSIPPI}

CANTON

N $32^{\circ} 36^{\prime}$

W $90^{\circ} 02^{\prime}$

$69 M$

LOCKHEED

Mississippi State

N $33^{\circ} 28^{\circ}$

W $88^{\circ} 48^{\circ}$

$122 M$

MS STATE Univ

1 ?

Pyranometer, Eppley pSp Microcom deC

(T)

Pyranometer, Eppley

MISSOURI

ST. Louts

EPA

Pyranometer, Eppley

Pyrgeometer, EPPLEY

PyrheliometER, Eppley

MONTANA

ANACONDA

N $46^{\circ} 07^{\prime}$

WI12.57'

$1625 \mathrm{M}$

FE

BILLINGS

iv $45^{\circ} 47^{\circ}$

W108.30

$950 \mathrm{M}$

FE
Pyranometer (T)

SILICON - SIMM - IR
Mag tape

$7 / 72$

STRIP CHART

LEEDS \& NORTH
$3 / 78-$
STRIP CHART

RUSTRACX RECORDER

(15)
STRIP CHART

RUSTRACK RFGoRDER

(15) 


\section{RADIOMETER}

TYPE, MFG, MODEL

\section{MONTANA}

(CONTD)

BOZEMAN

N $45^{\circ} 40^{\prime}$

W111 02 '

1449 M

FE

BROWNING

N $48^{\circ} 33^{\prime}$

W $113^{\circ} 01^{\prime}$

1360 M

FE

ButTE

N $46^{\circ}$

W112 $30^{\circ}$

1754 is

FE

Choteau

N $47^{\circ} 49^{\circ}$

'W112 $12^{\prime}$

$1158 \mathrm{M}$

FE

Colstrip

N $45^{\circ} 53^{\prime}$

W106 $38^{\prime}$

774 M

FE

DILLON

N $45^{\circ} 15^{\prime}$

W112 $38^{\circ}$

$1541 \mathrm{M}$

FE

ENNIS

N $45^{\circ} 21^{\prime}$

$W 111^{\circ} 44^{\prime}$

1502 M

FE
Pyranometer (T)

SILICON - SIMM - IR
Pyranometer. (T)

SILICON - SIMM - IR
Pyranometer (T)

SILICON - SIMM - IR

Pyranometer (T)

SILICON - SIMM - IR

Pyranometer (T)

SILICON - SIMM - IR
RECORDER

RECORD FORM
PERIOD OF

RECORD
STRIP CHART

RUSTRACK RECORDER

(I5)
1977 -

STRIP CHART

RUSTRACK RECORDER

(15)

STRIP CHART

RUSTRACK RECORDER

(15)

1977 -

1977 -
STRIP CHART RUSTRACK RECORDER

(15)

1977 -

STRIP CHART

RUSTRACK RECORDER

(15)

STRIP CHART

RUSTRACX RECORDER

(15)

STRIP CHART

RUSTRACX RECORDER

1977 - 
RADIOMETER

TYPE, MFG, MODEL

MONTANA

(CONTD)

FORT BENTON

N $47^{\circ} 48^{\prime}$

$W 110^{\circ} 40^{\prime}$

$792 \mathrm{M}$

FE

GLASGOW

N $48^{\circ} 12^{\prime}$

W106 $38^{\prime}$

$639 \mathrm{M}$

FE

GLENDIVE

N $47^{\circ} 07^{\prime}$

W $104^{\circ} 44^{\prime}$

$631 \mathrm{M}$

FE

GREAT FALLS

N $47^{\circ} 31^{\prime}$

WII1. $18^{\circ}$

$1015 \mathrm{M}$

FE

HAMILTON

iv $46^{\circ} 15^{\prime}$

W114'09'

$1097 \mathrm{M}$

FE

HARLOWTON

N $46^{\circ} 26^{\prime}$

$W 109^{\circ} 50^{\prime}$

$1270 \mathrm{M}$

FE

HAVRe

N 48. $34^{\prime}$

$W 109^{\circ} 40^{\prime}$

$758 \mathrm{M}$

FE

SILICON - SIMM - IR
Pyranometer (T)

SILICON - SIMM - IR
Pyranometer (T)

Pyranometer (T)

SILICON - SIMM - IR

Pyranometer (T)

SILICON - SIMM - IR
SILICON - SIMM - IR
RECORDER

RECORD FORM

PERIOD OF

RECORD
STRIP CHART 1977 -

Pyramometer (T)

RUSTRACK RECORDER

(15)

STRIP CHART

1977 -

Pyranometer (T)
Silicon - SIMM - IR

Pyranometer (T)

SILICON - SIMM - IR
STRIP. CHART

RUSTRACK RECORDER

(15)
STRIP CHART

RUSTRACK RECORDER

(15)
STRIP CHART

RUSTRACK RECORDER

(15)
1977 -

1977 -

1977 -

STRIP CHART

1977 -

RUSTRACK RECORDER

(15)

STRIP CHART

1977 -

RUSTRACX RECORDER

(15) 
RADIOMETER

TYPE; MFG, MODEL
RECORDER

RECORD FORM
PERIOD OF

RECORD

\section{MONTANA}

(CONTD)

Helena

N $46^{\circ} 35^{\prime}$

W112.02'

$1257 \mathrm{M}$

FE

JORDAN

N $47^{\circ} 19^{\prime}$

W106 $54^{\prime}$

853 M

FE

KALISPELL

N $48^{\circ} 13^{\prime}$

$W 114^{\circ} 16^{\prime}$

902 M

FE

LEWI STOWN

N $47^{\circ} 04^{\prime}$

$W 109^{\circ} 25^{\prime}$

$1207 \mathrm{M}$

FE

LIBBY

N $48^{\circ} 24^{\prime}$

$W 115^{\circ} 35^{\prime}$

$626 \mathrm{M}$

FE

LIVINGSTON

N $45^{\circ} 39^{\prime}$

W110 $34^{\prime}$

1369 м '

FE

Miles City

N $46^{\circ} 25^{\prime}$

W105'49'

$72 \mathrm{M}$

FE
Pyranometer (T)

SILICON - SIMM - IR

STRIP CHART

RUSTRACK RECORDER

(15)

Pyranometer (T)

SILICON - SIMM - IR

STRIP CHART

RUSTRACK RECORDER

(15)

Pyranometer (T)

SILICON - SIMH - IR

SIRIP CHART

RUSTRACK RECORDER

(15)

Pyranometer (T)

SILICON - SIMM - IR

STRIP CHART

RUSTRACX RECORDER

(15)

Pyranometer (T)

SILICON - SIMM - IR

STRIP CHART

RUSTRACK RECORDER

(15)

Pyranometer (T)

SILICON - SIMH - IR

STRIP CHART

RUSTRACK RECORDER

(15)

Pyranometer (T)

SILICON - SIMM - IR

STRIP CHART

RUSTRACK RECORDER

(15)
1977 -

1977. -

1977 -

1977 -

1977 -

1977 -

1977 - 


\begin{tabular}{|c|c|c|c|}
\hline i & $\begin{array}{l}\text { APPENDIX B } \\
\text { (CONTD) }\end{array}$ & . & \\
\hline STATION LOCATION & $\begin{array}{c}\text { RADIOMETER } \\
\text { TYPE, MFG. MODEL }\end{array}$ & $\begin{array}{l}\text { RECORDER } \\
\text { RECORD FORM }\end{array}$ & $\begin{array}{l}\text { PERIOD } 0 \\
\text { RECORD }\end{array}$ \\
\hline \multicolumn{4}{|c|}{$\frac{\text { MONTANA }}{\text { (CONTD) }}$} \\
\hline $\begin{array}{l}\text { MI SSOULA } \\
\text { N } 46^{\circ} 52^{\prime} \\
\text { W11 } \\
982 \mathrm{M} \\
\text { FE }\end{array}$ & $\begin{array}{l}\text { PyRanometer (T) } \\
\text { SILICON - SIMM - IR }\end{array}$ & $\begin{array}{l}\text { STRIP CHART } \\
\text { RUSTRACK RECORDER } \\
\text { (15) }\end{array}$ & 1977 - \\
\hline 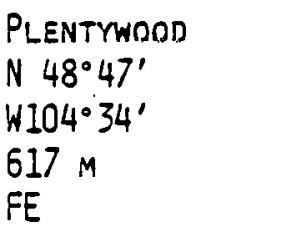 & $\begin{array}{l}\text { Pyranometer (T) } \\
\text { Silicon - SimM - IR }\end{array}$ & $\begin{array}{l}\text { STRIP CHART } \\
\text { RUSTRACK RECORDER } \\
\text { (15) }\end{array}$ & 1977 - \\
\hline $\begin{array}{l}\text { POLSON } \\
\text { N } 47^{\circ} 41^{\prime} \\
W 114^{\circ} 10^{\prime} \\
899^{\prime M} \\
\text { FE }\end{array}$ & $\begin{array}{l}\text { Pyranometer (T) } \\
\text { Sili ICON - SIMM - IR }\end{array}$ & $\begin{array}{l}\text { STRIP CHART } \\
\text { RUSTRACK RECORDER } \\
\text { (I5) }\end{array}$ & 1977 - \\
\hline $\begin{array}{l}\text { RED LODGE } \\
N 45^{\circ} 11^{\prime} \\
\text { N109.1 } \\
1691 \mathrm{M} \\
\text { FE }\end{array}$ & $\begin{array}{l}\text { Pyranometer (T) } \\
\text { Silicon - SIMM - IR. }\end{array}$ & $\begin{array}{l}\text { STRIP CHART } \\
\text { RUSTRACK RECORDER } \\
\text { (15) }\end{array}$ & 1977 - \\
\hline $\begin{array}{l}\text { SI DNEY } \\
N 47^{\circ} 42^{\prime} \\
\text { W104 } \\
5880^{\circ} \\
\text { FE }\end{array}$ & $\begin{array}{l}\text { PyRANOMETER (T) } \\
\text { SILICON }- \text { SIMM - IR }\end{array}$ & $\begin{array}{l}\text { STRIP CHART } \\
\text { RUSTRACK RECORDER } \\
\text { (15) }\end{array}$ & 1977 - \\
\hline $\begin{array}{l}\text { THOMPSON FALLS } \\
N 47^{\circ} 35^{\prime} \\
\text { W115 } \\
751 \text { M } \\
\text { FE }\end{array}$ & $\begin{array}{l}\text { PYRANOMETEA (T) } \\
\text { SILICON - SIMM - IR }\end{array}$ & $\begin{array}{l}\text { STRIF CHART } \\
\text { RUSTRACK RECORDER } \\
\text { (15). }\end{array}$ & $1977=$ \\
\hline $\begin{array}{l}\text { WEST YELLOWSTONE } \\
N 44^{\circ} 55^{\prime} \\
\text { W11 } \\
20311^{\circ} 04^{\prime} \\
\text { FE }\end{array}$ & $\begin{array}{l}\text { Pyranometer (T) } \\
\text { Silicon - SIMM - IR }\end{array}$ & $\begin{array}{l}\text { STRIP CHART } \\
\text { RUSTRACK RECORDER } \\
\text { (15) }\end{array}$ & $1977-$ \\
\hline
\end{tabular}


RADIOMETER .

TYPE, MFG. MODEL
RECORDER

RECORD FORM
PERIOD OF

RECORD

NEBRASKA

LINCOLN

Pyranometer, Eppley 10

$4 / 75-5 / 75$

LAMBda Instruments CORP, Junction \& L2-500

\section{NEVADA}

BOULDER CITY

N $35^{\circ} 59^{\circ}$

$W 114^{\circ} 5^{\prime}$

$762 \mathrm{M}$

UNIV of NV

ELDORADO

N $35^{\circ} 48^{\prime}$

$W 115^{\circ} 00^{\prime}$

$547 \mathrm{M}$

SCE

LAKE Mead

N 35'59'

$W 114^{\circ} 51^{\prime}$

$2525 \mathrm{M}$

USGS

Las Vegas

N $36^{\circ} 09^{\prime}$

$W 115^{\circ} 10^{\prime}$

$72 \mathrm{M}$

WEST

Las Vegas

N $36^{\circ} 08^{\prime}$

$W 115^{\circ} 17^{\prime}$

$667 \mathrm{M}$

LBL

RENO

N $39^{\circ} 40^{\circ}$

$W 119 \cdot 40^{\prime}$

$1514 \mathrm{M}$

UNIV OF NV

Pyranometer, Eppley-

(5)

$1952-1953$

LIGHTBULB TYPE, APPROX, 10

Mag tape (17)

1975 -

SPECTROSUN SR 75

(5)

$\begin{array}{lcl}\text { Pyranometer, EPPley. PSP } & \text { Charts \& digital } & 2 / 74- \\ \text { Pyrhel IOMeter, Eppley NIP } & (5) & 12 / 74-\end{array}$

Pyrheliometer, Eppley NIP

THERMOPILE JUNCTION

Pyranometer; Eppley

(17) : :

1977 -

Pyranometer, Eppley PSP Charts \& DIgITAl 4/74Pyrmeliometer, EPPLEY NIP (24)

Pyranometer, Eppley PSP Charts \& digital 4/74 Pyrhel IOMETER, EPpley NIP $(3,7)$ 


\section{APPENDIX B}

(CONTD)

STATION LOCATION

RADIOMETER

TYPE, MFG, MODEL

NEVADA

(CONTD)

\section{RENO}

N $39^{\circ} 30^{\circ}$

$W 119^{\circ} 47^{\prime}$

$1341 \mathrm{M}$

LBL
Pyranograph R401

\section{NEH_JERSEY}

Cartaret

us metals Refining Co.

JERSEY CITY

M. H. MEYerson \& Co., InC.

New BRUNSWICK

N $40^{\circ} 30^{\circ}$

W $74^{\circ} 28^{\prime}$

$45 \mathrm{M}$

RUTGERS UNIV

Princeton

RCA-LABS

SeABroOK

C. W. Thornthwaites Lab

TrEnTon

TWIN RIVERS

Princeton Univ
RECORDER

RECORD FORM

PERIOD OF

RECORD

(24)

$2 / 72-$

\section{NËL MEXICO}

Albuquerque

SANDia Labs

Albuguerque

LBL
Pyranometer, 'Eppley

STRIP CHART

(20)

$$
1949-1967
$$

$1949-1967$

STRIP CHART

$1969-1975$

Mag tare

Pyranometer, Eppley.pSP Mag tape .7/76-

Pyrheliometer, EPpley NIP

Pyranometer, Eppley pSp Digital mag tape 5/76-

Pyrheliometer, Radiometrics

CirCumsolar Telescope 


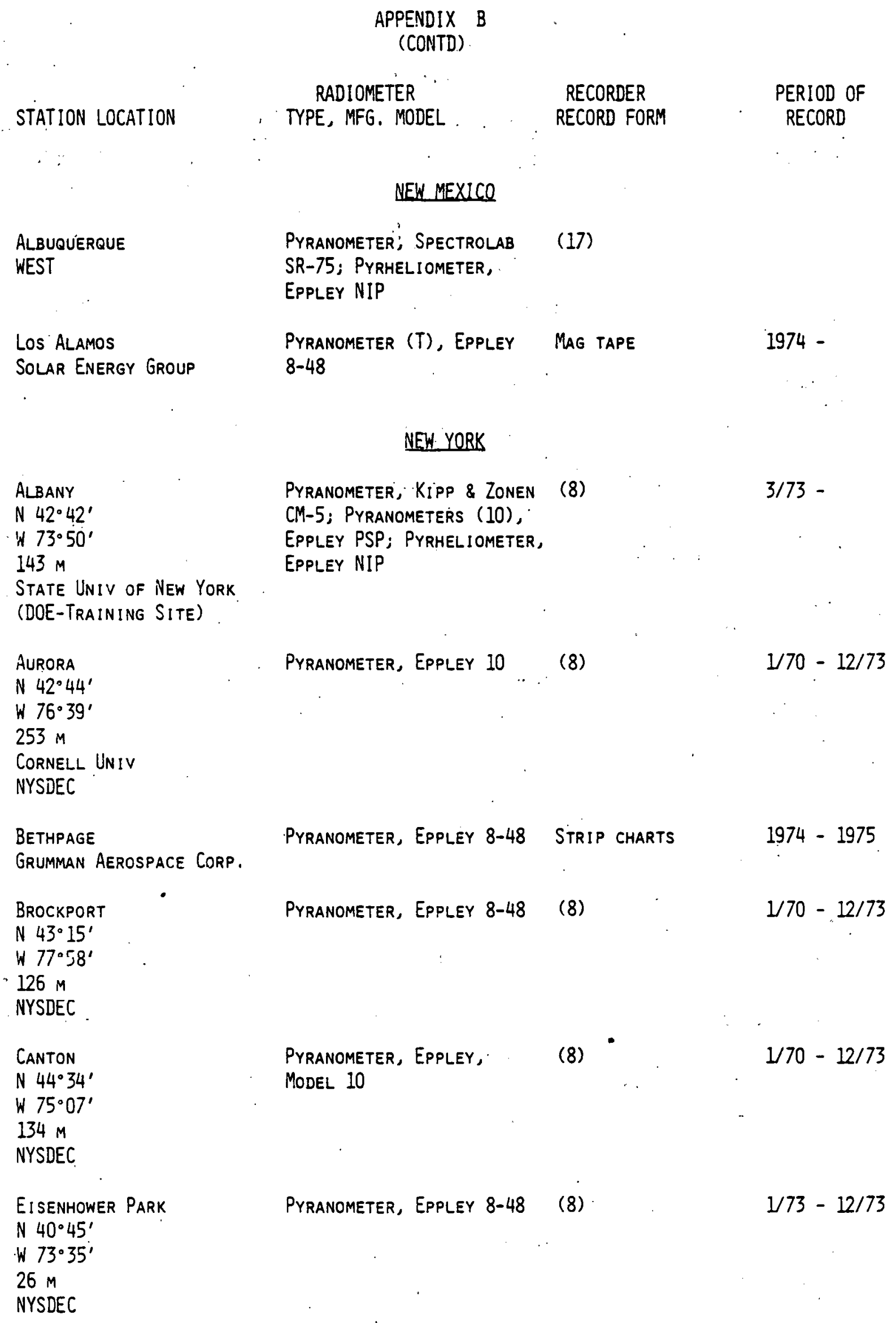




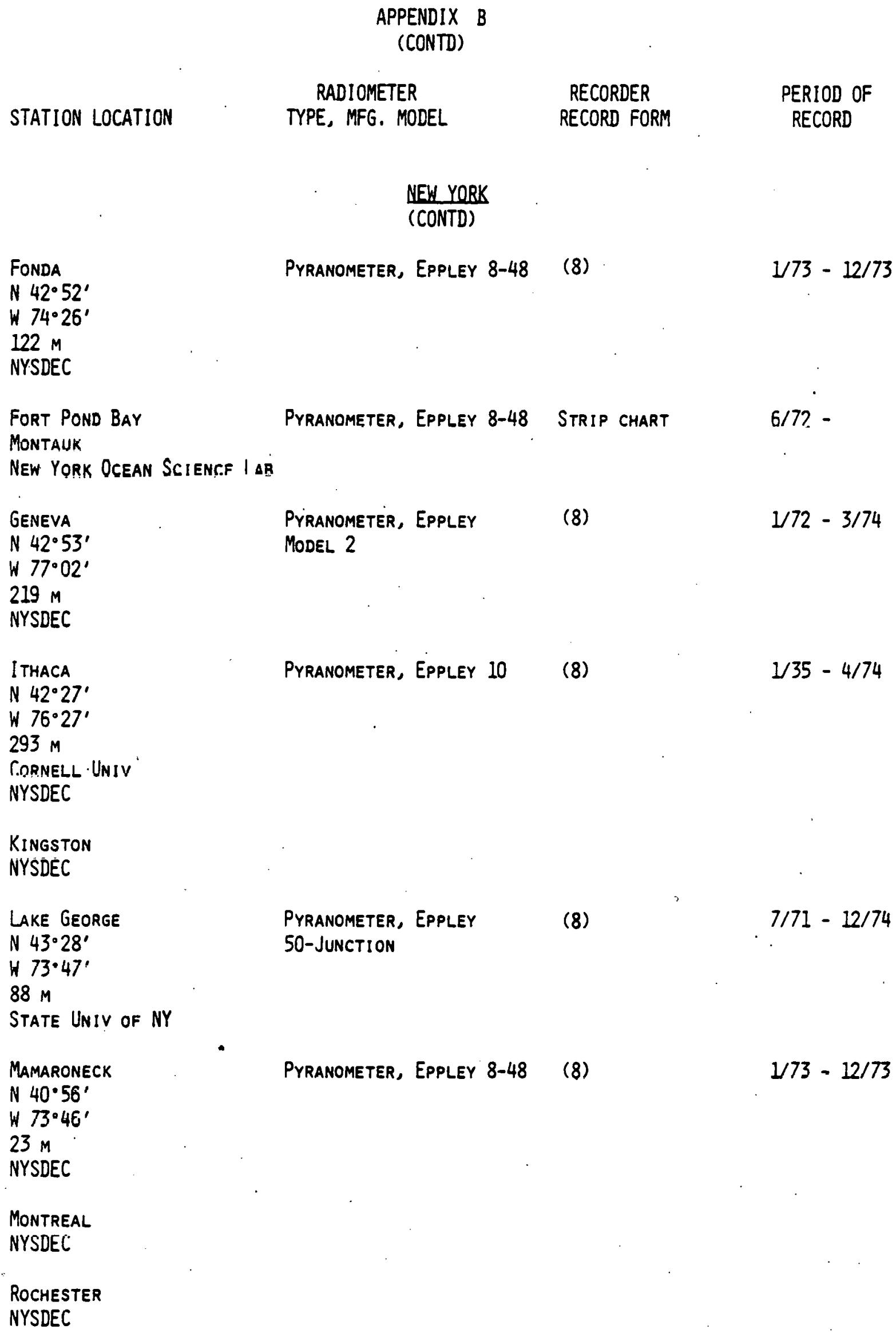

$1 / 73-12 / 73$

NEW York OCEan SCIEnTS.F I aB

GENEVA
$N 42^{\circ} 53$
$W 77^{\circ} 02^{\prime}$
219 M
NYSDEC

Pyranometer, Eppley

MOdEL 2

(8)

$1 / 72-3 / 74$

ITHACA

N $42^{\circ} 27^{\prime}$

W $76^{\circ} 27^{\prime}$

$293 \mathrm{M}$

C.ORNELL UNIV

NYSDEC

KINGSTON

NYSDEC

Lake George

Pyranometer, Eppley

Pyranometer, Eppley 10

(8)

$1 / 35-4 / 74$

N $43^{\circ} 28^{\prime}$

W 73.47'

SO-JUNCTION

(8)

$7 / 71-12 / 74$

$88 \mathrm{M}$

STATE UNIV OF NY

Mamaroneck

iN $40^{\circ} 56^{\prime}$

Pyranometer, Eppley 8-48

(8)

$1 / 73-12 / 73$

W $73^{\circ} 46^{\prime}$

$23 \mathrm{M}$

NYSDEC

Montreal

NYSDEC

ROCHESTER

NYSDEC 
STATION LOCATION

RADIOMETER

TYPE, MFG, MODEL

NEES YORK

(CONTD)

ROOSEVELT ISLAND

NYSDEC

SCHENECTADY

N $42^{\circ} 48^{\circ}$

W $73^{\circ} 56^{\prime}$

$69 \mathrm{M}$

NYSDEC

SYRACUSE

Suny College of

ENVIRONMENTAL SCIENCE

UPTON

N $40^{\circ} 52^{\prime}$

W $72^{\circ} 53^{\prime}$

$23 \mathrm{M}$

Brookhaven National Lab

Welfare IsLand

N $40^{\circ} 46^{\prime}$

W $73^{\circ} 56^{\circ}$

$8 \mathrm{M}$

NYSDEC

Whiteface MT

N $44^{\circ} 24^{\prime}$

W $73^{\circ} 52^{\prime}$

$604 \mathrm{M}$

ASRC

Whiteface MT LOdGe

NYSDEC

APPENDIX B

(CONTD)

Pyranometer, Eppley $8-48$

(8)

$1 / 73-12 / 73$

RECORDER

RECORD FORM

PERIOD OF

RECORD

SUMMER OF 1973

Pyranometer, Matrix Inc.

MARK I-G

Pyranometer, Eppley Strip chart $(8,22) 1950-1958$

50-JUNCTION;

Pyranometer, Eppley 8-48

1968 -

Pyranometer, Eppley 8-48 (8)

$1 / 73-12 / 73$

Pyranometer, Kipp \& Zonen (8)

$1 / 72-12 / 73$

CM-2-63

NORTH CAROLINA

ApEX

N $35^{\circ} 39^{\circ}$

W $78^{\circ} 01^{\prime}$

$87 \mathrm{M}$

Carolina Power \& Light Co

Pyranometer, Eppley 8-48 Westinghouse
Mode 1 WR-4C

Pyranometer, Eppley 8-48 Westinghouse
Mode 1 WR-4C

$4 / 73-$ 


\author{
RADIOMETER \\ TYPE, MFG. MODEL
}

RECORDER

RECORD FORM
PERIOD OF

RECORD

NORTH CAROLINA

(CONTD)

Camp Lejeune
RTI
CLINTON
RTI

Pyranometer, Eppley PSP Hourly Printouts 3/78 INTEGRATER

Pyranometer, EpPley PSP Hourly printouts 3/7.8 PYRHELIOMETER, EPPLEY NIP INTEGRATER ULTRAVIOLET, EPPLEY

DURHAM
NORTH CAROLINA/

TECHNical Research CTR

Pyranometer

STRIP CHART

$1974-$

PYRHFi.IOMFTER

Pyranometer, EPPLEY PSP

HOURLY PRINTOUTS $3 / 78$ -

JACKSONVILLE

RTI

INTEGRATER

Research Triangle Park

N $35^{\circ} 52^{\prime}$

W $78^{\circ} 45^{\prime}$

$132 \mathrm{M}$

EPA

SLOOP POINT

RTI

Pyranometer, EPPLey PSP HOURLy PRINTOUTS 3/78 -

Pyrheliometer, EPPLEY NIP;

UltRAVIOLET, EPPLEY

SOUTHPORT

N $33^{\circ} 58^{\prime}$

$W 78^{\circ} 01^{\prime}$

$8 \mathrm{M}$

Carolina Power \& Light

WALLACE

Pyranometer, Eppley 8048

Mag tape

WESTINGHOUSE

$1 / 75-$

RTI

Pyranometer, EPPLEY PSP

HOURLY PRINTOUTS

$3 / 78-$

WILMINGTUN

RTI

Pyranometer, Eppley PSP

INTEGRATER

HOURLY PRINTOUTS 3/78 -

INTEGRATER

OHIO

CINCINNATI

N $39^{\circ} 07^{\prime}$.

W $84^{\circ} 33^{\prime}$

HRL

'ERL-NOAA

Pyranometer, Eppley UV

STRIP CHART

(g)
9/5/67 - 6/69

BROKEN RECORD 


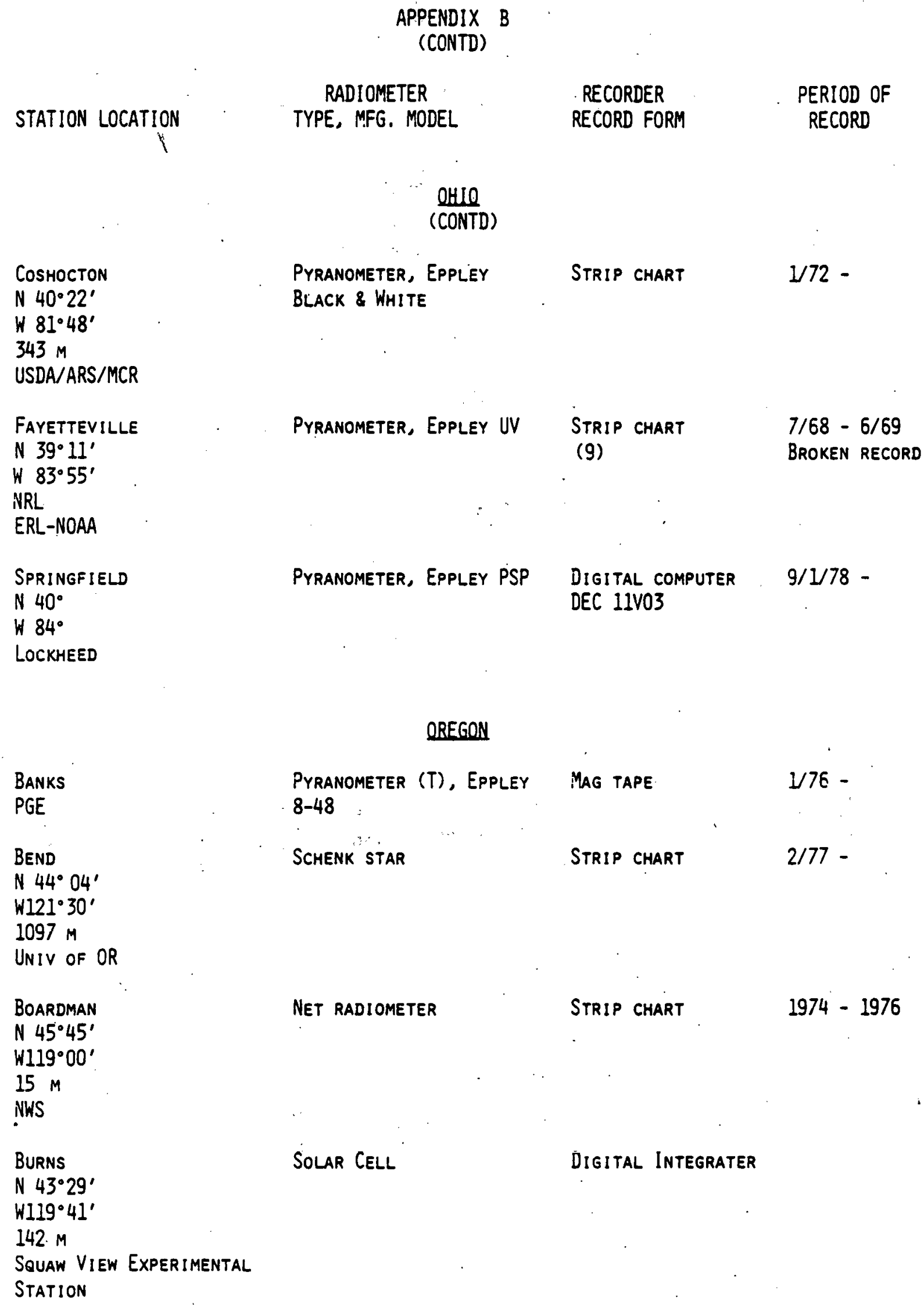




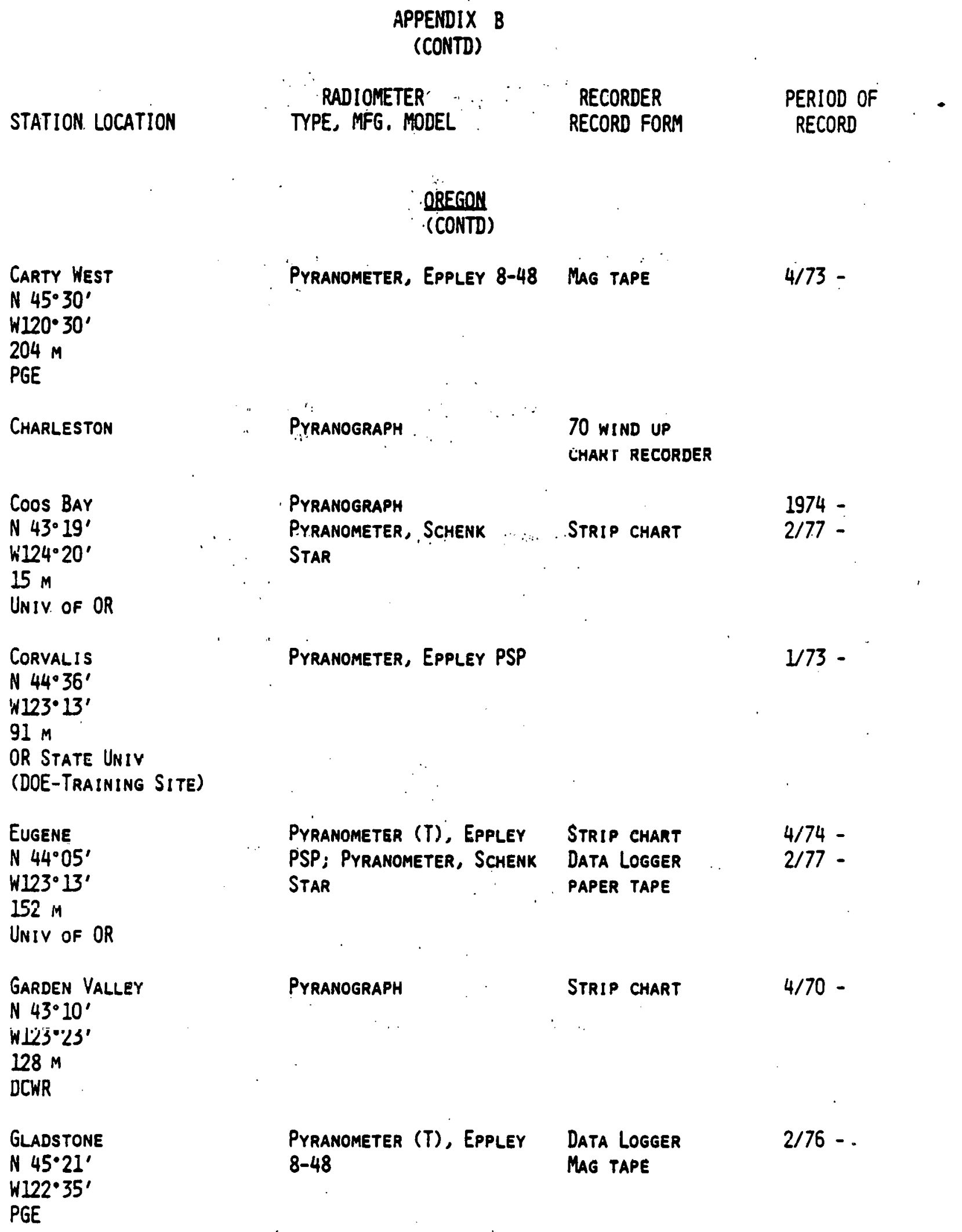


RADIOMETER

TYPE, MFG, MODEL.

\section{OREGON}

(CONTD)

Grant Pass

N $42^{\circ} 25^{\prime}$

$W 123^{\circ} 20^{\prime}$

$282 \mathrm{M}$

PPL

KLamath Falls

N $42^{\circ} 12^{\prime}$

$W 121^{\circ} 47^{\prime}$

$1250 \mathrm{M}$

LAGrande

N $45^{\circ} 19^{\prime}$

$W 118^{\circ} 05^{\prime}$

$847 \mathrm{M}$

UNIV OF OR

LAPINE

Pacific Northwest Bell co

LEBANON

MEDFORD

N $42^{\circ} 22^{\prime}$

$W 122^{\circ} 52^{\prime}$

$403 \mathrm{M}$

BLL

Melrose

N $43^{\circ} 10^{\prime}$

W123.30'

$228 \mathrm{M}$

DCWR

MyrtLe Creek

N 43.03'

$W 123^{\circ} 04^{\prime}$

$362 \mathrm{M}$

DCWR

Pyranograph

Pyranograph

\section{LINTRONIX}

Pyranograph

70 WINDUP

CHART RECORDER
PERIOD OF

RECORD
Data logger.

Mág tape

1976 -
Pyranometer; Schenk

Solar Cell

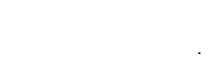

$\therefore \quad$ DATA LOGGER

\section{Chart recorom
Data Logger
Mag tape
$(5,28,31)$ \\ Chart recorom
Data Logger
Mag tape
$(5,28,31)$}

1176 -

$2 / 77-$

STRIP CHART

Chart

4/71 -

70 WIND UP
$\therefore$ CHART RECORDER

1971 - 
RADIOMETER

TYPE, MFG, MODEL

\section{OREGON}

(CONTD)

Pebble Springs

N $44^{\circ}$

$W 120^{\circ}$

228 M

PGE

Portland

N $45^{\circ} 30^{\prime}$

$H 122=40^{\circ}$

$61 \mathrm{M}$

PPL

PORTLAND

N $45^{\circ} 36^{\prime}$

$W 122^{\circ} 36^{\prime}$

$8 \mathrm{M}$

NWS

REDMOND

N $44^{\circ} 10^{\prime}$

W121.

914 iM

NWS

SAINT HELENS

ReICHHOLd Chemical Inc.

SALEM

N $44^{\circ} 55^{\prime}$

W123.

$76 \mathrm{M}$

PGE

TIGARD

N $42^{\circ} 25^{\prime}$

$W 122^{\circ} 45^{\prime}$

PPL

WHI TEHORSE RANCH

N $42^{\circ} 20^{\prime}$

$W 118^{\circ} \cdot 14^{\prime}$

$1280 \mathrm{M}$

UNIV OF OR $8-48$

Pyranograph

Pyranograph

Pyranograph

Pyranometer (T), Eppley

8-48

LINTRONIX (T)

Pyranometer, Schenk
RECORDER

RECORD FORM
PERIOD OF

RECORD

Pyranometer, Eppley 8-48 Data Logger 1/74 -

Mag tape

Pyramometer (T), Eppley Data logger $1976^{\circ}$ -

Mag tape

CHART (28)

1974 -

CHART (28)

CHART (28)

70 WIND UP.

$4 / 77$ -

CHART RECORDER

Data Logger

Mag tape (28)

$11 / 77-$

Data logier

$1975=$

Mag Tape

Chart

$2 / 77-$ 


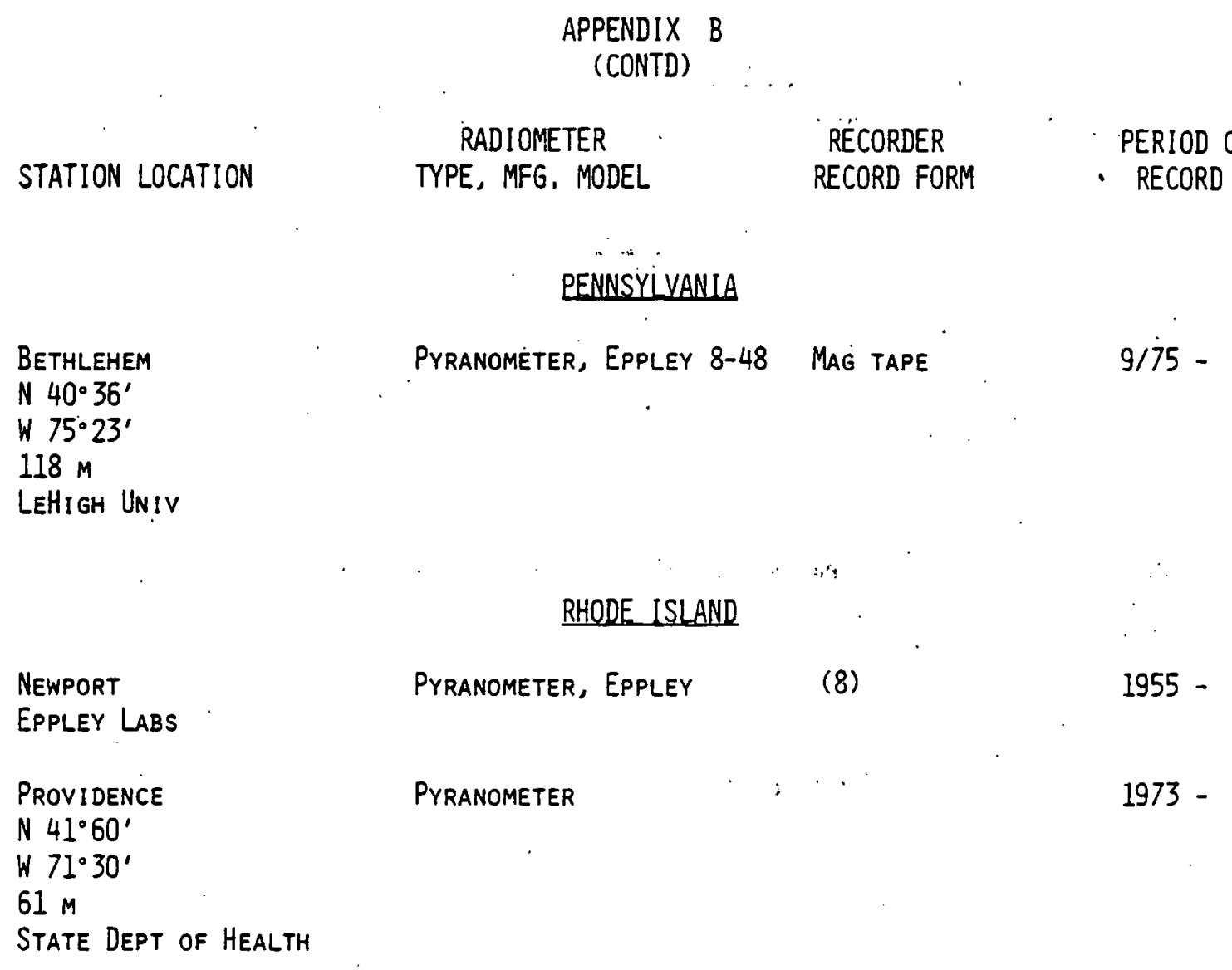

SOUTH CAROLINA

A! KEN

N $33^{\circ} 17^{\prime}$

W $81^{\circ} 42^{\prime}$

$44 \mathrm{M}$

Savannah River Lab.

BLACKVILLE

N $33^{\circ} 22^{\prime}$

W $81^{\circ} 19^{\prime}$

$99 \mathrm{M}$

CLEMSON UNIV

CLEMSON

N $34^{\circ} 41^{\prime}$

W $82^{\circ} 49^{\circ}$

$250 \mathrm{M}$

CLEMSON UNIV

HARTSVILLE

N $34^{\circ} 24^{\prime}$

W $80^{\circ} 10^{\prime}$

$72 \mathrm{M}$

Carolina Power \& light co

Pyranograph, BeLFort - Dailiy RECORD $1968-1976$

Pyranograph, Belfort DaILY Record

$1964-1976$

(10)

$8 / 74$ (10)

Pyranometer, Eppley 8-48 Mag tape 5/75 - . 
RADIOMETER

TYPE, MFG, MODEL

SOUTH CAROLINA

(CONTD)

Pontiac

N $34^{\circ} 08^{\prime}$

W $80^{\circ} 52^{\prime}$

$134 \mathrm{M}$

Clemson Univ

PyRÁnOgRaph, BELFort
RECORDER

RECORD FORM
PERIOD OF

RECORD

DAILY RECORD

$1965-1976$

\section{IENNESSEE}

CLINTON

N. $36^{\circ} 01^{\prime}$

W $84^{\circ} 11^{\prime}$

$317 \mathrm{M}$

TVA

Cumberland

N $36^{\circ} 23^{\prime}$

W $87^{\circ} 38^{\prime}$

$200 \mathrm{M}$

TVA

DAISY

N $35^{\circ} 13^{\prime}$

W $85^{\circ} 06^{\prime}$

$227 \mathrm{M}$

TVA

HARTSVILLE

N $36^{\circ} 21^{\prime}$

W $86^{\circ} 06^{\prime}$

$171 \mathrm{M}$

TVA

KINGSPORT

N $36^{\circ} 31^{\prime}$

W $82^{\circ} 32^{\prime}$

367 M

ASG INDUSTRIES, INC.
(10)

Mag tape nova 12001969 -

HEWLETT PACKARD 345
Pyranometer, Eppley 8-48 Mag tape Nova 12001971 -

PYRRADIOMETER

HEWLETT PACKARD 345
Pyranometer, Eppley 8-48 Mag tape Núva 1200 . 1971 -

PYRRADIOMETER

HeWLetT PACKARd 345
Pyranometer, Eppley 8-48 Mag tape nova 1200 1/78-

PyrRadiometer 
RADIOMETER

TYPE, MFG. MODEL
RECORDER

RECORD FORM
PERIOD OF

RECORD

\section{IENMESSEE}

(CONTD)

KINGSTON

N $35^{\circ} 54^{\prime}$

W $84^{\circ} 31^{\prime}$

$260 \mathrm{M}$

TVA

New Johnsonville

N $36^{\circ} 01^{\prime}$

W $87^{\circ} 59^{\prime}$

$120 \mathrm{M}$

TVA

OAK RIDGE

N $36^{\circ} 01^{\prime}$

W $84^{\circ} 14^{\prime}$

$287 \mathrm{M}$

NOAA/ATDL

PHIPPS BEND

N $36^{\circ} 29^{\prime}$

W $82^{\circ} 49^{\prime}$

$233 \mathrm{M}$

TVA

WATTS BAR

N $35^{\circ} 35^{\prime}$

$W 84^{\circ} 47^{\circ}$

$217 \mathrm{M}$

TVA PYRRADIOMETER PYRRADIOMETER
Pyranometer, Eppley $8-48$

PyRRadiometer
Mag tape nova 1200

Hewlett Packard 345
Late 1975 -
Pyranometer, Eppley 8-48 Mag tape Nova 1200
Hewlett Packard 345

Pyranometer, Eppley 8-48 Mag tape Nova 1200
Hewlett Packard 345

$12 / 75-$

\begin{tabular}{|c|c|c|}
\hline SOLAMETER, LITRONIC & MAg TAPE & $10 / 71-11 / 73$ \\
\hline LIMITED & & \\
\hline $\begin{array}{l}\text { PYRANOMETER, EPPLEY } \\
\text { PYRHEL IOMETER }\end{array}$ & $\begin{array}{l}\text { STRIP CHART } \\
\text { MAG TAPE }\end{array}$ & $\begin{array}{l}1953- \\
177-\end{array}$ \\
\hline
\end{tabular}

Pyranometer, Eppley 8-48 Mag tape Nova 1200 i/78HEWLETT PACKaro $345^{\circ}$

Pyranometer, Eppley 8-48 Mag tape llova 12001973 HEWLETT PACKARD $345^{\circ}$

\section{IEXAS}

College Station

Pyranometers (2) (S) (T), Hourly totals EPPLEY PSP

3 JAN - 12 JUL 1973

N $30^{\circ} 36^{\prime}$

$W 96^{\circ} 20^{\prime}$

$17 \mathrm{M}$

TX A \& M UNIV 


$\begin{array}{ccc}\text { APPENDIX B } & \\ \text { (CONTD) } & \\ \text { STATION LOCATION } & \text { RADIOMETER } & \text { RECORDER } \\ \text { TYPE, MFG. MODEL } & \text { RECORD FORM } & \text { PERIOD OF } \\ \text { RECORD }\end{array}$

IEXAS

(CONTD)

FORT HOOD

N $31^{\circ} 04^{\prime}$

W $97^{\circ} 24^{\prime}$

$244 \mathrm{M}$

LBL

SAN ANTONIO

TRINITY UNIV

(JOE - Training Site)

WACO

BAYLOR UNIV

LOGAN

N $41^{\circ} 45^{\prime}$

W $111^{\circ} 50^{\prime}$

$1487 \mathrm{M}$

UT STATE UNIV
Pyranometer, Eppley PSP

Pyrhel Iometer, Radio-

METRICS CIRCUMSOLAR

TELESCOPE

'Pyranometers (6), Eppley PSP: Pyahel IORLTER3 (2), EPPLEY NIP

Pyranometer, Eppley Model II

UITAH

Pyranometer, KipP 8. ZONEN CM-3
Mag tape

$7 / 76-$

VIRGINIA

HAMPTON

N $37^{\circ} 05^{\prime}$

W $76^{\circ} 22^{\prime}$

$24 \mathrm{M}$

Langley Research CTR

WALLOPS IsLAND

WALLOPE FLIGIIT Center (NASA)

WARRENTON

Pyranometers, Hy-cal, INTERTECHNOLOGY CORP,

STRIP CHART

$6 / 68^{\circ}-$
TECHTRONIC

Weather Measure

CHARTS \& TAPE

$2 / 75-$ 


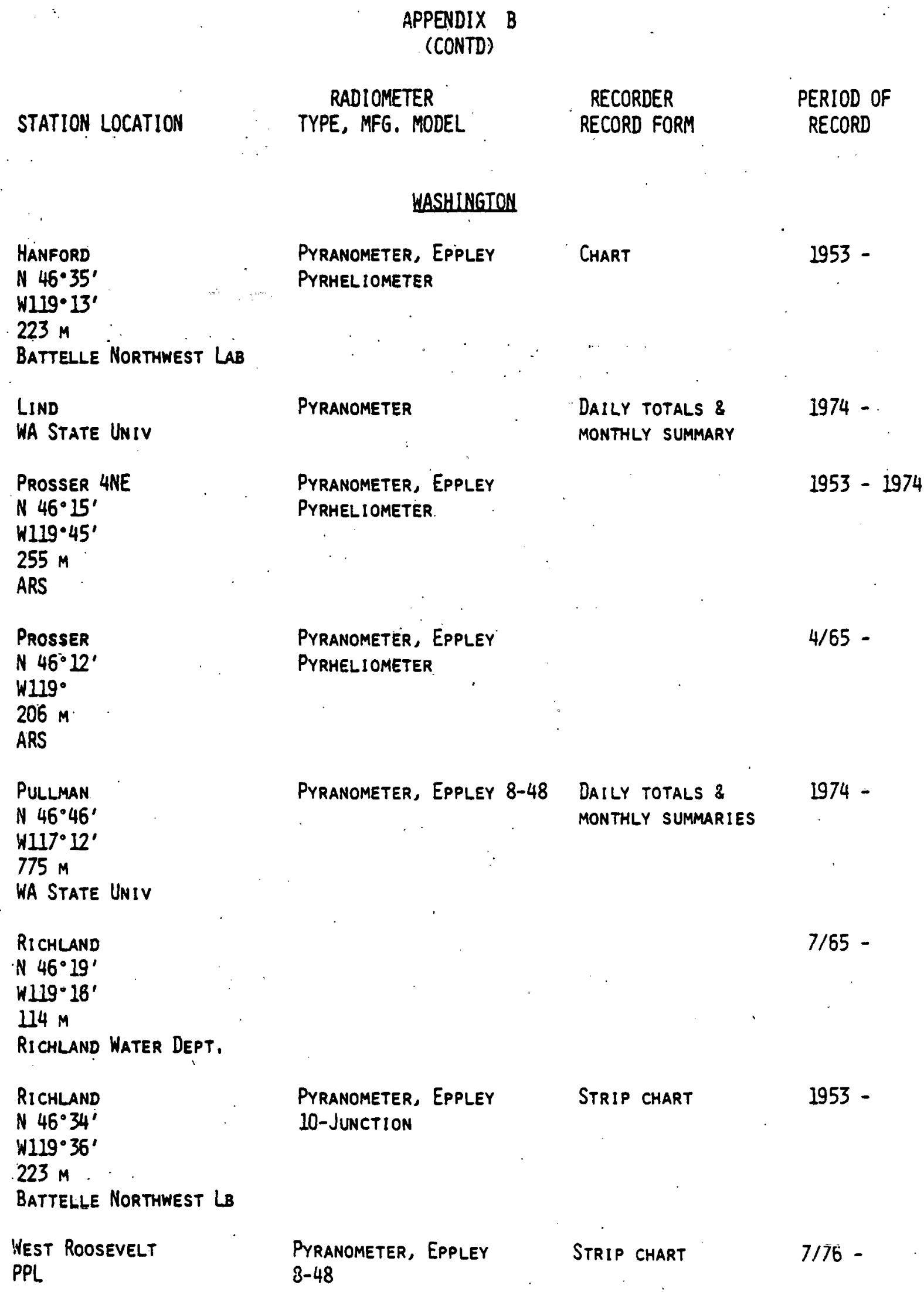


RADIOMETER

TYPE, MFG. MODEL
RECORDER

RECORD FORM
PERIOD OF

RECORD
PARSONS

N $39^{\circ} 06^{\prime}$

W $79^{\circ} 39^{\prime}$

509. $M$

USFS

Madison

UNIV OF WI

Cheyenne

N $41^{\circ} 08^{\prime}$

$W 104^{\circ} 49^{\prime}$

$1876 \mathrm{M}$

IVEST

LARAMIE

N $41^{\circ} 19^{\prime}$

$W 105^{\circ} 36^{\prime}$

$2195 \mathrm{M}$

UNIV OF WY

ROCK SPRINGS

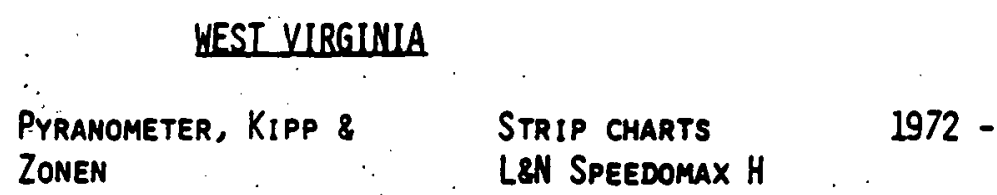

\section{WEST VIRGIMIA}

\section{WSCONSIN}

Pyramometer (3), (T), fluxe Data logger $3 / 77$ PSP; SPECTROLAB, BLACK \& (12)

WHITE

WOMING

Pyranometer, Spectrolab Mag tape $(5,17) \quad 1977$ SR 75

Pyranometer, Eppley

$1 / 78-$

PyRhELIOMETER, EPPLEY

Pyranometer, Matrix, Inc. Mag tape

$8 / 71-$ 
APPENDIX B

(CONTD)

\section{RADIOMETER}

STATION LOCATION
TYPE, MFG. MODEL

GOREIGN

\section{ANTARTICA}

SOUTH POLE

AMUNDSEN-SCOTT

GMCC

Eppley; Pyrheliometers

(2), Eppley:

\section{RECORDER}

RECORD FORM
PERIOD OF

RECORD

CANADA

Montreal, Que,

Ministry of Transport

Pyranometers (4) (S) (D), Mag tape

Canadian Meteorological Service

OtTawa, Ont.

TORONTO

(RES, STA.)

TORONTO

(SCARBOROUGH)

\section{PAMAMA CAMAL ZONE}

BALBOA

iN $9^{\circ}$

W $79^{\circ} 30^{\prime}$

SRL

Pyranometer, Eppley PSP

(1)

$3 / 73-12 / 77$

SAMOA

SAMOA

S $14^{\circ} 15^{\prime}$

W170 $34^{\prime}$

GMCC
$(8,30)$

$(8,30)$

$(8,29,30)$

$(8,30)$ 
APPENDIX C

PUBLICATIONS WITH SOLAR RADIATION DATA

1. Solar Radiation Measurements, 1968 - 1977, Smithsonian Radiation Biology LABORATORY, ROCKVILLE, MD.

2. Auburn University, Micrometeorological Data, Agricultural Weather Series-No. 11, 12, 13, 14 AND 15.

3. Solar Radiation and Sunshines Data for the Southwestern United States, 1966 - 1977, the State of Arizona Solar Energy Research Commission and State Climatologist FOR ARI ZONA.

4. Compilation Sheets of Solar Radiation Data from Desert Sunshine Exposure tests, INC., PHOENIX, ArizONa.

5. Solar padiation Measurements in California, State of California Resources Agency, DEPARTMENT of Water REsources,

6. A Floridian's Guide to Solar Energy, State Energy Office, University of Florida and Florida Solar Energy Center.

7. Nevada's Weather and Climate, Houghton, Sakamoto, and Gifford, Special Publication 2, 1975, Nevada Bureau of Mines and Geology, Mackay School of Mines, UniVERSITY OF NeVADA, Reno.

8. Solar Energy Atlas for New York State, Atmospheric Sciences Research Center, State University of New York at Albany.

9. Tabulated Data Sheets, National Radiation laboratory, erl-noaA, Boulder, co,

10. NWS-CLEmson University ANd S.C. Agricultural Experiment Stations=Climatological. DATA, 1966-1967-1968-1969-1973-1974-1975-1976, PLUS' TABULATED SHEETS, 19651970-1971-1972, fOR PONTIAC AND CLEMSON AND 1970-1971-1972, fOR BLACKVILLE.

11. Tabulated Data Sheets, The Marine lab, University of Guam, Agana, Guam,

12. Solar Radiation Reception, Probabilities, and Areal Distribution in the NorthCentral Region. North Central Regional Research Publication 225, Technical

- Bulletin 300, 1975. Agricultural Experiment Station, University of Minnesota.

13. Climate of Minnesota, Part VI, Solar Radiation at St. Paul. Technical Bulletin 280-1971, Agricultural Experiment Station, University of Minnesota. 
APPENDIX C

(CONDT)

\section{PUBLICATIONS WITH SOLAR RADIATION DATA}

14. Data Sheets from Logan, Utah, Jänuary 1972.

15. Solar Insolation Data compiled by Fowlkes Engineering, 1820 South Seventh Avenue, BOZEMAN, MT 59715.

16. Solar Radiation Data for San Diego County, San:Diego Gas \& Electric Co., and San Diego State University, Department of Mechanical. Engineering.

17. The West Associates Sini ar Resource Evaluation Projeet, 3ponsured by Suuthern California Edison Research and Development, P.0. Box 800, Rosemead, Ca 91770.

18. Mean Solar Radiation Data for.florida Cities, Florida Solar Energy Center, 300 State Road 401, Cape Canaveral, Fl 32920.

19. Solar Radiation in Peru during Joint-11: A Guide for Modelus, Bigelow laboratory for OCEan Sciences, West Boothbay Harbor, Maine 04575.

20. Solar fadiation at New Brunswick, New Jersey, New Jersey Agricilltural Experiment Station, Cook College, Rutgers University - The State University of New Jersey, NEW BRUNSWICK.

21. Solar Data Users horkshop abstracts of Papers and Notes, The University of New Mexico, College of Engineering,

22. Climatology of Brookhaven national laboratory 1974 through 1977, Associated Universities, Inc., United States Department of Energy.

23. Redstone Arsenal, Huntsville, al, Solar Radiation data, May 1976 through September 1978, The Johnson Environmental \& Energy Center, UAH.

24. California Solar Data Manual, March 1978, Lawrence Berkeley laboratory, University of California, Berkeley, Ca 94720.

25. Avekalie Daily Solar Radiation for Oak Ridge, TN, 1956 through 1975. Atmospheric Turbulence anjo Diffusion labonatory national Oceanic and Atmospheric administraTION, OAK RIDGE, TN 37830.

26. The Hawai: Natural Energy institute, Solar Radiation Measurements in Hawail, Takeshi Yoshihara and Paul C. Ekern, September 1, 1977, under the auspices of the hawal! Natural Energy Institute, University of Hawal!, Honolulu, Hawali. 
APPENDIX C

(CONTD)

PUBLICATIONS GITH SOLAR RADIALION DATA

27. Environmental data for Sites in the Mational Solar Data Network, National Solar Heating and Cooling Demonstration Program, us Department of Energy, by ibM féderal Systems Division, Command and Space Systems, 150 Sparkman Drive, Huntsville, Alabama 35805.

28. Input Data for Solar Systems, November 1978, prepared for Department of Energy ay the US Department of Commerce, nOAa, Environmental Data and information Service, national Climatic Center, Asheville, NC.

29. Observation of Direct Solar Radiation and atmospheric turbidity at TorontoScarborough from 1960 to 1970, J. R. Latimer, Camadian Meteorological Research Reports, Environment Canada -Atmospheric Environment Service, 4905 Dufferin Street, Downsview, Ontario.

30. Monthly Radiation Summary, Canadian Atmospheric Service, Imformation Canada, OTTAWA.

31. Monthly Summary Solar Radiation Data, us Department of Commerce, noaA, edis, national Climatic Center, Asheville, NC.

32. Annual Report of the Solar Heating Experiment on the Grover Cleveland School, Boston, Massachusetts, conducted for the Energy Research and Development AdminiSTRATION. 
DEPARTMENT OF ENERGY NATIONAL SOLAR HEATING AND COOLING DEMONSTRATION SITES:

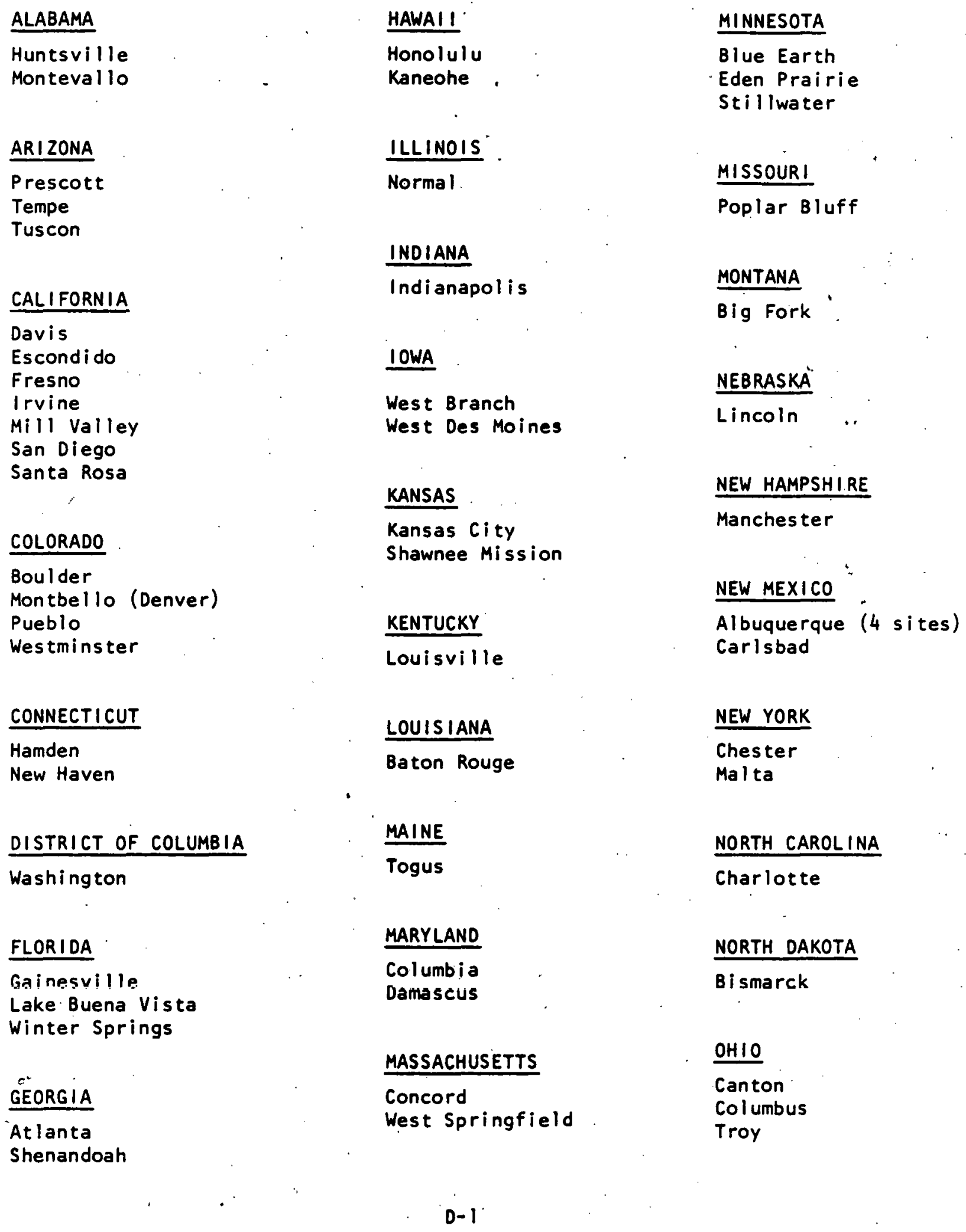


OKLAHOMA

El Reno

OREGON

Coos Bay

PENNSYLVANIA

West Chester

RHODE ISLAND .

James town

SOUTH CAROLINA

China Grove

Columbia

Greenville

Greenwood

St. Mat thews

SOUTH DAKOTA

Keys tone
TEXAS

Austin (2 sites)

San Antonio. (2 sites)

VIRGINIA

Leesburg

Lynchburg

Virginià Beach

VERMONT

Waitsfield

WASHINGTON

Richland

Seattle

WEST VIRGINIA

Charles Town

WISCONSIN

Mi lwaukee

Rice Lake 


\section{APPENDIX $\varepsilon$ \\ QUESTIONNAIRE TO IDENTIFY SOLAR RADIATION DATA}

If you have solar radiation data, please complete this form and mail to:

Universtey of Alabama in Huntsville

Johnson Environental and Energy Center

P.0. Box 1247

Huntguille, Alabama 35807

Aten: E. A. Carter

Dace

Locacton where solar radiation data was recorded: 1at. $N$

(Use separate form for each sensor)

long. $W$

Instrument elevation: mol feet or

meters

Description of recording alte (urban, rural, grass, roof top, horizontal surface, etc.):

Perlods of record and hours of operation:

Station history (change of location, instruments, etc.):

Sensor type

Type of data: direct

Frequency (minute, hourly.

Recorder type

Callbration-Englneerling data: (for sensor and recorder)

Data format (tape, str1p, c1rcular charts-digitized hourly or dally?): Manuf aceúrer 8lobal da1ly, etc.): Model Inclined

Is data avallable? What form? Serip Charts?

For what perlod? past future

Are records properly annotated and sultable for microfilming?

Collateral meteorological daca avallable:

Published daca avallable:

W111 you release the data to the National Climatic Center for use in Solar Energy Projecta?

Reporting organization and name of custodlan of records:

Malling eddrese:

Telephone number: FTS:

E-1 